\title{
Boronated Enynes as Versatile Sources of Stereodefined and Skeletally Diverse Molecules
}

\author{
Alain Hercouet, Fabienne Berrée, Chia Hui Lin, Loïc Toupet and Bertrand Carboni* \\ Sciences Chimiques de Rennes, UMR 6226 et Groupe Matière condensée et Matériaux, UMR 6626 \\ CNRS-Université de Rennes 1, Campus de Beaulieu, 35042 Rennes CEDEX, France.
}

\section{Supporting Information}

I. General information S-2

$\begin{array}{lll}\text { II. Synthesis of boron-substituted enynes } 1 & \text { S-2 }\end{array}$

III. General procedure for the palladium-catalyzed cycloisomerisation of $\mathbf{1}$ S-6

IV. Cycloisomerisation/Diels-alder cycloaddition S-8

V. General procedure for palladium-catalyzed cycloisomerisation /Diels-Alder $\begin{array}{ll}\text { cycloaddition/allylboration sequence } & \text { S-9 }\end{array}$

VI. Metathesis/Diels-Alder/oxidation sequence S-14

$\begin{array}{ll}\text { VII. Platinum-catalyzed cycloisomerisation of 1a } & \text { S-15 }\end{array}$

$\begin{array}{lll}\text { VIII. X-ray structure of } 5 & \text { S-16 }\end{array}$

$\begin{array}{ll}\text { IX. Reproductions of }{ }^{1} \mathrm{H} \text { and }{ }^{13} \mathrm{C} \text { spectra } & \mathrm{S}-17\end{array}$ 


\section{I- General information}

All reactions were carried out with anhydrous solvents. Toluene and tetrahydrofuran were distilled from sodium/benzophenone and methylene chloride from $\mathrm{P}_{2} \mathrm{O}_{5} .{ }^{1} \mathrm{H}$ NMR spectra $(300 \mathrm{MHz})$ and ${ }^{13} \mathrm{C}$ NMR (75 MHz) were recorded on a Bruker $\mathrm{AC} 300$ spectrometer, in $\mathrm{CDCl}_{3}$ solutions with $\mathrm{Me}_{4} \mathrm{Si}$ as internal reference. Chemical shifts are given in ppm and coupling constants $J$ in Hz. Elemental analyses were performed by the Microanalytical Laboratory at the Centre Regional de Mesures Physiques de l'Ouest and high-resolution mass spectra (HRMS) recorded on a Varian MAT 311 spectrometer or a high resolution MS/MS ZABSpec TOF Micromass spectrometer. Analytical thin layer chromatography was performed on Merck Silica Gel 60 F254 plates.

\section{II- Synthesis of boron-substituted enynes 1}

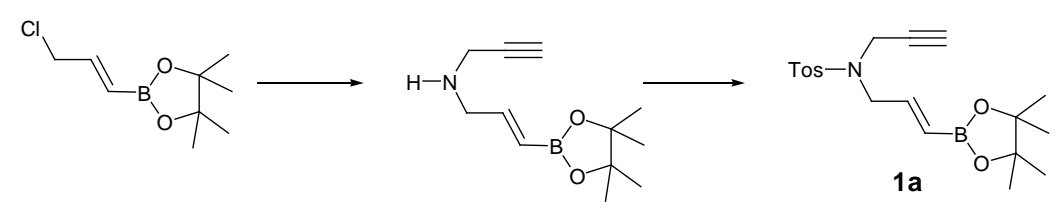

To a solution of (E)-2-(3-chloro-1-propenyl)-4,4,5,5-tetramethyl-1,3,2-dioxaborolane (0.789 g, 3.9 mmol) in $\mathrm{CH}_{3} \mathrm{CN}(10 \mathrm{~mL})$ was added $\mathrm{K}_{2} \mathrm{CO}_{3}(1.08 \mathrm{~g}, 7.8 \mathrm{mmol})$ and propargylamine $(660 \mathrm{mg}, 820$ $\mu \mathrm{L}, 12 \mathrm{mmol})$. The reaction mixture was stirred at room temperature during three days. Diethylether $(10 \mathrm{~mL})$ was then added and the precipitate was removed by filtration through celite. The filtrate was concentrated in vacuo. Excess of amine was eliminated by distillation (bp $50-60^{\circ} \mathrm{C}, 0.1 \mathrm{~mm} \mathrm{Hg}$ ).

${ }^{1} \mathrm{H}-\mathrm{NMR}\left(300 \mathrm{MHz}, \mathrm{CDCl}_{3}\right) \delta 6.62(\mathrm{dt}, J=18.1,5.3 \mathrm{~Hz}, 1 \mathrm{H}), 5.61(\mathrm{dt}, J=18.1,1.5 \mathrm{~Hz}, 1 \mathrm{H}), 3.35-$ $3.42(\mathrm{~m}, 4 \mathrm{H}), 2.20(\mathrm{t}, J=2.3 \mathrm{~Hz}, 1 \mathrm{H}), 1.25(\mathrm{~s}, 12 \mathrm{H}) ;{ }^{13} \mathrm{CNMR}\left(75 \mathrm{MHz}, \mathrm{CDCl}_{3}\right) \delta 150.1,121.2(\mathrm{br})$, 82.6, 81.4, 71.0, 41.3, 36.9, 24.7.

To a solution of this crude aminoboronate in dry $\mathrm{CH}_{2} \mathrm{Cl}_{2}(20 \mathrm{~mL})$ was added dropwise at $0^{\circ} \mathrm{C}$ Net 3 $(557 \mu \mathrm{L}, 4 \mathrm{mmol})$ and a solution of $p$-toluenesulfonyl chloride $(0.76 \mathrm{~g}, 4 \mathrm{mmol})$ in $\operatorname{dry~} \mathrm{CH}_{2} \mathrm{Cl}_{2}(5 \mathrm{~mL})$. The reaction mixture was stirred during $12 \mathrm{~h}$ at room temperature and washed with water $(2 \times 10 \mathrm{~mL})$. The organic phase was dried over $\mathrm{MgSO}_{4}$, filtered and concentrated in vacuo. The product was purified by recrystallisation from ethanol.

$1 \mathrm{a}(700 \mathrm{mg}, 48 \%), \mathrm{mp} 86^{\circ} \mathrm{C}$ (ethanol). ${ }^{1} \mathrm{H}-\mathrm{NMR}\left(300 \mathrm{MHz}, \mathrm{CDCl}_{3}\right) \delta 7.75(\mathrm{~d}, J=8.1 \mathrm{~Hz}, 2 \mathrm{H}), 7.30$ $(\mathrm{d}, J=8.3 \mathrm{~Hz}, 2 \mathrm{H}), 6.47$ (dt, $J=17.9,5.7 \mathrm{~Hz}, 1 \mathrm{H}), 5.70(\mathrm{dt}, J=17.9,1.5 \mathrm{~Hz}, 1 \mathrm{H}), 4.10(\mathrm{~d}, J=2.4 \mathrm{~Hz}$, 2H), $3.91(\mathrm{dd}, J=5.7,1.3 \mathrm{~Hz}, 2 \mathrm{H}), 2.43(\mathrm{~s}, 3 \mathrm{H}), 2.00(\mathrm{t}, J=1.3 \mathrm{~Hz}, 1 \mathrm{H}), 1.27(\mathrm{~s}, 12 \mathrm{H}) .{ }^{13} \mathrm{C}-\mathrm{NMR}(75$ 
$\left.\mathrm{MHz}, \mathrm{CDCl}_{3}\right) \delta 145.8,143.5,136.1,129.5,127.7,123.3$ (br), 83.4, 76.4, 73.8, 49.9, 36.1, 24.7, 21.5. MS (EI) calcd. For $\mathrm{C}_{18} \mathrm{H}_{23} \mathrm{NO}_{4}{ }^{11} \mathrm{BS}\left[\mathrm{M}-{ }^{-} \mathrm{CH}_{3}\right]^{+}: 360.1440$, found: 360.1452 .

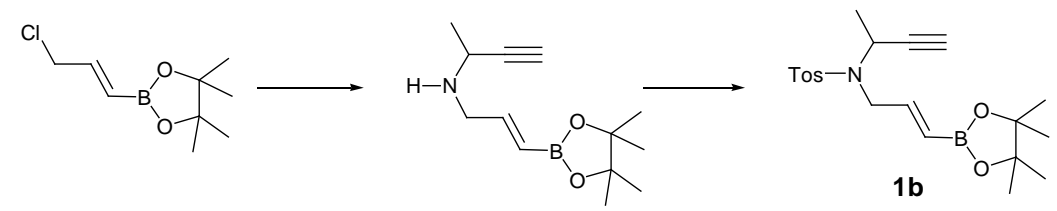

To a solution of (E)-2-(3-chloro-1-propenyl)-4,4,5,5-tetramethyl-1,3,2-dioxaborolane (2 g, $10 \mathrm{mmol})$ in $\mathrm{CH}_{3} \mathrm{CN}(20 \mathrm{~mL})$ was added $\mathrm{Net}_{3}(7 \mathrm{~mL}, 50 \mathrm{mmol})$ and but-3-yn-2-amine, hydrochloride $(2.10 \mathrm{~g}, 20$ $\mathrm{mmol})$. The reaction mixture was stirred at room temperature during three days. Diethylether $(20 \mathrm{~mL})$ was then added and the resulting precipitate was removed by filtration through celite. The filtrate was concentrated in vacuo. Excess of amines was eliminated by distillation (bp 50-60 ${ }^{\circ} \mathrm{C}, 0.1 \mathrm{~mm} \mathrm{Hg}$ ). ${ }^{1} \mathrm{H}-\mathrm{NMR}\left(200 \mathrm{MHz}, \mathrm{CDCl}_{3}\right) \delta 6.45(\mathrm{dt}, J=17.8,7.0 \mathrm{~Hz}, 1 \mathrm{H}), 6.08(\mathrm{~d}, J=17.7 \mathrm{~Hz}, 1 \mathrm{H}), 3.31-3.59$ (m, $3 \mathrm{H}), 2.28(\mathrm{t}, J=2.1 \mathrm{~Hz}, 1 \mathrm{H}), 1.44(\mathrm{~d}, J=7.4 \mathrm{~Hz}, 3 \mathrm{H}), 1.28(\mathrm{~s}, 12 \mathrm{H})$.

To a solution of this crude aminoboronate in dry $\mathrm{CH}_{2} \mathrm{Cl}_{2}(30 \mathrm{~mL})$ was added dropwise at $0^{\circ} \mathrm{C} \mathrm{Net}_{3}$

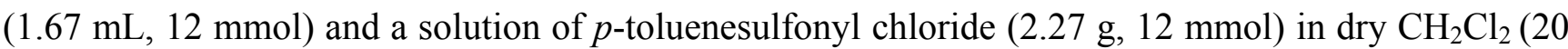
$\mathrm{mL})$. The reaction mixture was stirred during $12 \mathrm{~h}$ at room temperature and washed with water $(2 \times 10$ $\mathrm{mL}$ ). The organic phase was dried over $\mathrm{MgSO}_{4}$, filtered and concentrated in vacuo. The product was purified by silica gel chromatography (cyclohexane/Ethyl acetate: 6/1).

$1 \mathbf{b}(2.1 \mathrm{~g}, 54 \%)$. White solid, mp $132-133^{\circ} \mathrm{C}$ (diisopropylether). ${ }^{1} \mathrm{H}-\mathrm{NMR}\left(300 \mathrm{MHz}, \mathrm{CDCl}_{3}\right) \delta 7.73$ (d, $J=7.9 \mathrm{~Hz}, 2 \mathrm{H}), 7.29$ (d, $J=7.8 \mathrm{~Hz}, 2 \mathrm{H}), 6.65(\mathrm{dt}, J=17.9,5.3 \mathrm{~Hz}, 1 \mathrm{H}), 5.68(\mathrm{~d}, J=18.1 \mathrm{~Hz}, 1 \mathrm{H})$, $4.88(\mathrm{q}, J=6.9 \mathrm{~Hz}, 1 \mathrm{H}), 4.00(\mathrm{dd}, J=18.3,3.6 \mathrm{~Hz}, 1 \mathrm{H}), 3.79(\mathrm{dd}, J=17.4,6.1 \mathrm{~Hz}, 1 \mathrm{H}), 2.42(\mathrm{~s}, 3 \mathrm{H})$, $2.11(\mathrm{~s}, 1 \mathrm{H}), 1.43(\mathrm{~d}, J=6.6 \mathrm{~Hz}, 3 \mathrm{H}), 1.27(\mathrm{~s}, 12 \mathrm{H}) ;{ }^{13} \mathrm{C}-\mathrm{NMR}\left(75 \mathrm{MHz}, \mathrm{CDCl}_{3}\right) \delta 149.9,143.4$, 136.2, 129.4, 127.7, 83.3, 81.2, 73.3, 48.5, 46.3, 24.8, 22.7, 21.5. MS (EI) calcd. For $\mathrm{C}_{19} \mathrm{H}_{25} \mathrm{NO}_{4}{ }^{11} \mathrm{BS}$ $\left[\mathrm{M}-\mathrm{CH}_{3}\right]^{+}: 374.15974$, found: 374.1589 .

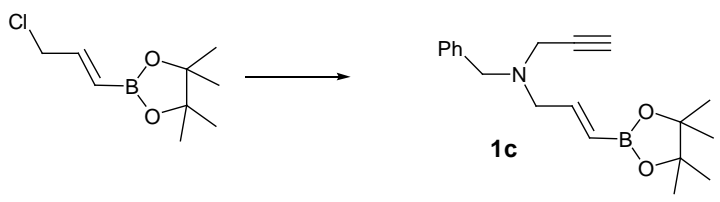

The reaction mixture from (E)-2-(3-chloro-1-propenyl)-4,4,5,5-tetramethyl-1,3,2-dioxaborolane (1.01 g, $5 \mathrm{mmol}$ ), $\mathrm{N}$-benzylprop-2-yn-1-amine (800 mg, $5.5 \mathrm{mmol}$ ), and $\mathrm{K}_{2} \mathrm{CO}_{3}\left(1.97 \mathrm{~g}, 15 \mathrm{mmol}\right.$ ) in $\mathrm{CH}_{3} \mathrm{CN}$ 
$(10 \mathrm{~mL})$ was stirred during three days at room temperature. The solid was removed by filtration through celite and washed with ether. The filtrate was concentrated in vacuo. The residue was purified through silica gel chromatography (cyclohexane/Ethyl acetate: 4/1).

1 c $(720 \mathrm{mg}, 46 \%) .{ }^{1} \mathrm{H}-\mathrm{NMR}\left(300 \mathrm{MHz}, \mathrm{CDCl}_{3}\right) \delta$ 7.39-7.23 (m, 5H), $6.66(\mathrm{dt}, J=18.0,5.5 \mathrm{~Hz}, 1 \mathrm{H})$, $5.75(\mathrm{~d}, J=18.0 \mathrm{~Hz}, 1 \mathrm{H}), 3.67(\mathrm{~s}, 2 \mathrm{H}), 3.32(\mathrm{~s}, 2 \mathrm{H}), 3.27$ (d, $J=5.6 \mathrm{~Hz}, 2 \mathrm{H}), 2.26(\mathrm{~s}, 1 \mathrm{H}), 1.28$ (s, 12H). ${ }^{13} \mathrm{C}-\mathrm{NMR}\left(75 \mathrm{MHz}, \mathrm{CDCl}_{3}\right) \delta 150.3,138.4,128.8,128.1,127.0,121.2$ (br), 83.0, 78.3, 73.2, 57.35, 57.3, 41.4, 24.6. MS (EI) calcd. For $\mathrm{C}_{19} \mathrm{H}_{26} \mathrm{NO}_{2}{ }^{11} \mathrm{~B}[\mathrm{M}]^{+}: 311.2056$, found: 311.2052 .
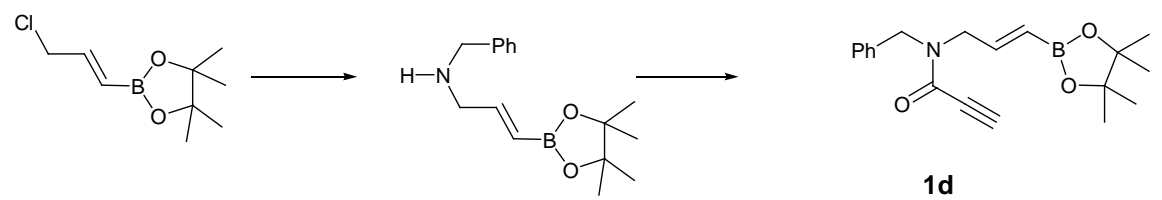

$\left(2^{E}\right)$-N-benzyl-3-(4,4,5,5-tetramethyl-1,3,2-dioxaborolan-2-yl)prop-2-en-1-amine was first prepared as previously described in the synthesis of $1 \mathbf{a}$ using benzylamine instead of propargylamine. ${ }^{1} \mathrm{H}-\mathrm{NMR}\left(200 \mathrm{MHz}, \mathrm{CDCl}_{3}\right) \delta$ 7.45-7.15 (m, 5H), $6.64(\mathrm{dt}, J=18.0,5.3 \mathrm{~Hz}, 1 \mathrm{H}), 5.65(\mathrm{dt}, J=18.1$, $1.5 \mathrm{~Hz}, 1 \mathrm{H}), 3.78(\mathrm{~s}, 2 \mathrm{H}), 3.35(\mathrm{dd}, J=5.3,1.5 \mathrm{~Hz}, 2 \mathrm{H}), 1.27(\mathrm{~s}, 12 \mathrm{H})$.

To a solution of this crude boronate $(740 \mathrm{mg}, 2.7 \mathrm{mmol})$ in dry $\mathrm{CH}_{2} \mathrm{Cl}_{2}(10 \mathrm{~mL})$ was first added at $0^{\circ} \mathrm{C}$ a solution of propiolic acid $(180 \mu \mathrm{L}, 2.9 \mathrm{mmol})$ in dry $\mathrm{CH}_{2} \mathrm{Cl}_{2}(5 \mathrm{~mL})$ and then DCC $(634 \mathrm{mg}, 3$ mmol) and DMAP (37 mg, $0.3 \mathrm{mmol})$. The reaction mixture was stirred overnight at room temperature. The resulting solid was removed by filtration through celite and the filtrate was concentrated in vacuo. The residue was purified by silica gel chromatography (cyclohexane/Ethyl acetate: $1 / 4$ to $1 / 1)$.

1d (450 mg, 51\%) as a mixture of two rotamers (1/1). ${ }^{1} \mathrm{H}-\mathrm{NMR}\left(300 \mathrm{MHz}, \mathrm{CDCl}_{3}\right) \delta$ 7.24-7.38 (m, $5 \mathrm{H}), 6.51(\mathrm{dt}, J=18.0,5.0 \mathrm{~Hz}, 1 \mathrm{H})$ and $6.47(\mathrm{dt}, J=18.1,5.2 \mathrm{~Hz}, 1 \mathrm{H}), 5.62(\mathrm{~d}, J=18.0 \mathrm{~Hz}, 1 \mathrm{H})$ and $5.52(\mathrm{~d}, J=18.1 \mathrm{~Hz}, 1 \mathrm{H}), 4.78(\mathrm{~s}, 2 \mathrm{H})$ and $4.60(\mathrm{~s}, 2 \mathrm{H}), 4.17(\mathrm{dd}, J=5.2,1.3 \mathrm{~Hz}, 2 \mathrm{H})$ and $4.01(\mathrm{dd}, J$ $=5.0,1.3 \mathrm{~Hz}, 2 \mathrm{H}), 3.14(\mathrm{~s}, 1 \mathrm{H})$ and $3.10(\mathrm{~s}, 1 \mathrm{H}), 1.31(\mathrm{~s}, 12 \mathrm{H})$ and $1.29(\mathrm{~s}, 12 \mathrm{H}) .{ }^{13} \mathrm{C}-\mathrm{NMR}(75 \mathrm{MHz}$, $\left.\mathrm{CDCl}_{3}\right) \delta 153.5$ and 153.1, 145.9 and 145.4, 135.9 and 135.6, 128.6 and 128.5, 128.2 and 127.8, 127.5 and 127.4, 122.3 (br), 83.4 and 83.2, 79.2 and 79.1, 75.5 and 75.4, 51.7 and 51.2, 46.8 and 46.7, 24.6. MS (EI) calcd. For $\mathrm{C}_{19} \mathrm{H}_{24} \mathrm{NO}_{3}{ }^{11} \mathrm{~B}[\mathrm{M}]^{+}: 325.1849$, found: 325.1876 . 


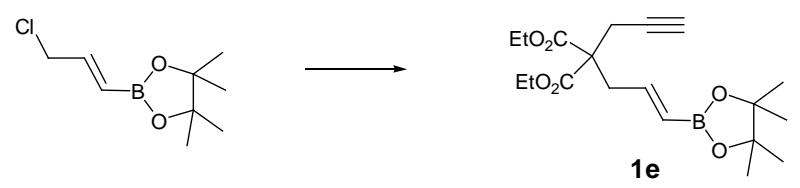

To a suspension of $\mathrm{NaH}(120 \mathrm{mg}, 3 \mathrm{mmol})$ in dry $\mathrm{THF}(5 \mathrm{~mL})$ at $0^{\circ} \mathrm{C}$ was added diethyl propargylmalonate $(489 \mathrm{mg}, 3 \mathrm{mmol})$ in THF $(5 \mathrm{~mL}) .{ }^{1}$ After stirring for $30 \mathrm{~min},(E)$-2-(3-chloro-1propenyl)-4,4,5,5-tetramethyl-1,3,2-dioxaborolane $(607 \mathrm{mg}, 3 \mathrm{mmol}$ ) was added and the reaction was stirred additional $15 \mathrm{~h}$. After addition of a saturated ammonium chloride solution $(5 \mathrm{~mL})$, the mixture was extracted with diethylether, and dried over $\mathrm{MgSO}_{4}$. The solvent was removed in vacuo and the crude product was by distillation (bp $100-105^{\circ} \mathrm{C} / 0.1 \mathrm{~mm} \mathrm{Hg}$ ).

$\mathbf{1}^{\mathrm{e}}(830 \mathrm{mg}, 76 \%) .{ }^{1} \mathrm{H}-\mathrm{NMR}\left(300 \mathrm{MHz}, \mathrm{CDCl}_{3}\right) \delta 6.34(\mathrm{dt}, J=17.8,6.3 \mathrm{~Hz}, 1 \mathrm{H}), 5.61(\mathrm{~d}, J=17.8 \mathrm{~Hz}$, $1 \mathrm{H}), 4.21-4.10(\mathrm{~m}, 4 \mathrm{H}), 2.85(\mathrm{~d}, J=7.3 \mathrm{~Hz}, 2 \mathrm{H}), 2.75(\mathrm{~d}, J=2.5 \mathrm{~Hz}, 2 \mathrm{H}), 1.97(\mathrm{t}, J=2.5 \mathrm{~Hz}, 1 \mathrm{H})$, $1.20(\mathrm{t}, J=7.3 \mathrm{~Hz}, 6 \mathrm{H}), 1.20(\mathrm{~s}, 12 \mathrm{H}) .{ }^{13} \mathrm{C}-\mathrm{NMR}\left(75 \mathrm{MHz}, \mathrm{CDCl}_{3}\right) \delta 169.6,146.3,83.2,78.8,71.5$, 61.7, 56.5, 38.4, 24.8, 22.7, 14.0. MS (EI) calcd. For $\mathrm{C}_{18} \mathrm{H}_{26} \mathrm{O}_{4}{ }^{11} \mathrm{~B}\left[\mathrm{M}-\mathrm{CH}_{3}\right]^{+}$: 349.1822, found : 349.1797.
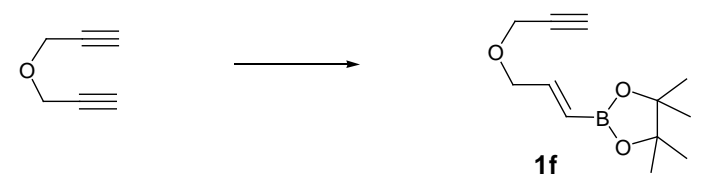

A $50 \mathrm{~mL}$ round bottom flask equipped with a septum inlet was charged with borane/dimethylsulfide complex (10M, $0.5 \mathrm{~mL}, 5 \mathrm{mmol})$ and dry THF $(2 \mathrm{~mL})$ under an argon atmosphere. $\alpha$-Pinene $(1.67 \mathrm{~mL}$, $10 \mathrm{mmol}$ ) was then added dropwise at $0^{\circ} \mathrm{C}$ and the mixture was stirred for $10 \mathrm{~min}$ followed by $2 \mathrm{~h}$ at room temperature. To the resulting suspension cooled to $-40^{\circ} \mathrm{C}$ was added dropwise a solution of the diyne $(1.88 \mathrm{~g}, 20 \mathrm{mmol})$ in $\mathrm{THF}(5 \mathrm{~mL})$. The mixture was stirred at $0^{\circ} \mathrm{C}$ for $1 \mathrm{~h}$ and further stirred at room temperature for additional $4 \mathrm{~h}$. It was cooled back to $0^{\circ} \mathrm{C}$ again prior to the addition of freshly distilled acetaldehyde $(4 \mathrm{~mL})$. The solution was warmed up and stirred for $15 \mathrm{~h}$ before removal of volatiles in vacuo. Diethylether $(20 \mathrm{~mL})$ were added, followed by pinacol $(5.3 \mathrm{~g}, 4.5 \mathrm{mmol})$. After $5 \mathrm{~h}$ at room temperature, the solvent was evaporated and the residue dissolved in pentane, washed twice with water and dried with $\mathrm{MgSO}_{4}$. The crude product was purified by distillation $\left(\mathrm{bp} 75-80^{\circ} \mathrm{C} / 1 \mathrm{~mm}\right.$ $\mathrm{Hg})$.

1f $(0.65 \mathrm{~g}, 65 \%) .{ }^{1} \mathrm{H}-\mathrm{NMR}\left(300 \mathrm{MHz}, \mathrm{CDCl}_{3}\right) \delta 6.63(\mathrm{dt}, J=18.2,4.9 \mathrm{~Hz}, 1 \mathrm{H}), 5.73(\mathrm{dt}, J=18.2,1.5$ $\mathrm{Hz}, 1 \mathrm{H}), 4.21-4.12(\mathrm{~m}, 4 \mathrm{H}), 2.43(\mathrm{t}, J=2.3 \mathrm{~Hz}, 1 \mathrm{H}), 1.26(\mathrm{~s}, 12 \mathrm{H}) .{ }^{13} \mathrm{C}-\mathrm{NMR}\left(75 \mathrm{MHz}, \mathrm{CDCl}_{3}\right) \delta$

\footnotetext{
${ }^{1}$ Brummond, K. M.; Chen, H.; Sill, P.; You, L. J. Am. Chem. Soc., 2002, 124, 15186.
} 
148.0, 83.3, 79.5, 74.5, 71.1, 57.4, 24.7. MS (EI) calcd. For $\mathrm{C}_{11} \mathrm{H}_{16} \mathrm{O}_{3}{ }^{11} \mathrm{~B}\left[\mathrm{M}-\mathrm{CH}_{3}\right]^{+}:$207.1192, found: 207.1197.

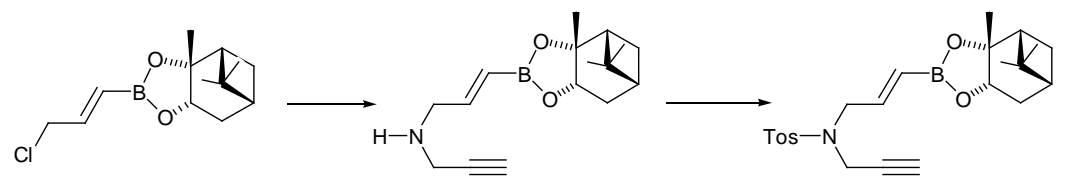

$1 \mathrm{~g}$

The same sequence as for 1a was carried out starting from the corresponding $(+)$-pinanediol derivative instead of (E)-2-(3-chloro-1-propenyl)-4,4,5,5-tetramethyl-1,3,2-dioxaborolane pinacol. The residue was purified by silica gel chromatography (cyclohexane/Ethyl acetate: $1 / 5$ to $1 / 1$ ).

1 g (510 mg, 31\%). ${ }^{1} \mathrm{H}-\mathrm{NMR}\left(300 \mathrm{MHz}, \mathrm{CDCl}_{3}\right) \delta 7.75(\mathrm{~d}, J=8.3 \mathrm{~Hz}, 2 \mathrm{H}), 7.31(\mathrm{~d}, J=8.2 \mathrm{~Hz}, 2 \mathrm{H})$, $6.48(\mathrm{dt}, J=18.0,5.7 \mathrm{~Hz}, 1 \mathrm{H}), 5.72(\mathrm{~d}, J=18.0 \mathrm{~Hz}, 1 \mathrm{H}), 4.32(\mathrm{dd}, J=8.8,1.6 \mathrm{~Hz}, 1 \mathrm{H}), 4.11(\mathrm{~d}, J=2.3$ Hz, 2H), $3.92(\mathrm{dd}, J=5.7,0.8 \mathrm{~Hz}, 2 \mathrm{H}), 2.44(\mathrm{~s}, 3 \mathrm{H}), 2.39-2.20(\mathrm{~m}, 2 \mathrm{H}), 2.07(\mathrm{t}, J=5.7 \mathrm{~Hz}, 1 \mathrm{H}), 2.02$ $(\mathrm{t}, J=2.4 \mathrm{~Hz}, 1 \mathrm{H}), 1.95-1.84(\mathrm{~m}, 2 \mathrm{H}), 1.41(\mathrm{~s}, 3 \mathrm{H}), 1.31(\mathrm{~s}, 3 \mathrm{H}), 1.12(\mathrm{~d}, J=10.6 \mathrm{~Hz}, 1 \mathrm{H}), 0.86(\mathrm{~s}$, 3H). ${ }^{13} \mathrm{C}-\mathrm{NMR}\left(75 \mathrm{MHz}, \mathrm{CDCl}_{3}\right) \delta 145.7,143.6,136.0,129.5,127.7,122.7$ (br), 85.9, 77.8, 76.4, 73.9, 51.3, 50.0, 39.4, 38.1, 36.1, 35.4, 28.6, 27.1, 26.4, 24.0, 21.5. MS (EI) calcd. For $\mathrm{C}_{16} \mathrm{H}_{23} \mathrm{NO}_{2}{ }^{11} \mathrm{~B}$ $[\mathrm{M}-\mathrm{Ts}]^{+}: 272.1822$, found : 272.1828. $[\alpha]_{\mathrm{D}}{ }^{20}=+9.6^{\circ}\left(\mathrm{c} 1, \mathrm{CH}_{2} \mathrm{Cl}_{2}\right)$.

\section{III- General procedure for the palladium-catalyzed cycloisomerisation of 1.}

In a Schlenk flask, tris(dibenzylideneacetone)dipalladium(0)-chloroform adduct (5.2 mg, $0.05 \mathrm{mmol}$, $2.5 \% \mathrm{~mol})$, tri-o-tolylphosphine $(3.0 \mathrm{mg}, 0.05 \mathrm{mmol}, 5 \% \mathrm{~mol})$, acetic acid $(0.6 \mu \mathrm{L}, 0.01 \mathrm{mmol}, 5 \%$ $\mathrm{mol})$ and toluene $(1 \mathrm{~mL})$ were stirred for $15 \mathrm{~min}$ at room temperature. Enyne $1(0.2 \mathrm{mmol})$ was added and the mixture was kept $2 \mathrm{~h}$ at room temperature under an argon atmosphere, except for $\mathbf{1 c}$ and $\mathbf{1 b}$, respectively $18 \mathrm{~h}$ at room temperature and $2 \mathrm{~h}$ at $50^{\circ} \mathrm{C}$. Attempts to purify compounds 2 by chromatography on silica gel resulted in partial decomposition that explained that the NMR data of dienes $\mathbf{2}$ were extracted from the NMR spectra of the crude mixtures.

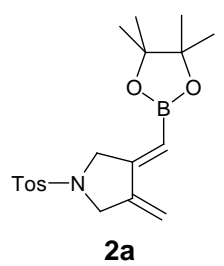


${ }^{1} \mathrm{H}-\mathrm{NMR}\left(300 \mathrm{MHz}, \mathrm{CDCl}_{3}\right) \delta 7.74(\mathrm{~d}, J=8.1 \mathrm{~Hz}, 2 \mathrm{H}), 7.33(\mathrm{~d}, J=8.1 \mathrm{~Hz}, 2 \mathrm{H}), 5.75(\mathrm{t}, J=2.3 \mathrm{~Hz}$, $1 \mathrm{H}), 5.52(\mathrm{t}, J=2.2 \mathrm{~Hz}, 1 \mathrm{H}), 5.02(\mathrm{~s}, 1 \mathrm{H}), 4.23(\mathrm{~d}, J=2.3 \mathrm{~Hz}, 2 \mathrm{H}), 3.97(\mathrm{t}, J=2.2 \mathrm{~Hz}, 2 \mathrm{H}), 2.43(\mathrm{~s}$, $3 \mathrm{H}), 1.28(\mathrm{~s}, 12 \mathrm{H}) .{ }^{13} \mathrm{C}-\mathrm{NMR}\left(75 \mathrm{MHz}, \mathrm{CDCl}_{3}\right) \delta 154.2,143.7,142.5,132.8,129.7,127.9,125.3$, $107.3,83.5,53.4,52.4,24.8,21.5$.

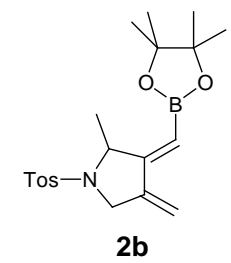

${ }^{1} \mathrm{H}-\mathrm{NMR}\left(300 \mathrm{MHz}, \mathrm{CDCl}_{3}\right) \delta 7.70(\mathrm{~d}, J=8.1 \mathrm{~Hz}, 2 \mathrm{H}), 7.36(\mathrm{~d}, J=8.1 \mathrm{~Hz}, 2 \mathrm{H}), 5.65(\mathrm{t}, J=2.4 \mathrm{~Hz}$, $1 \mathrm{H}), 5.45(\mathrm{t}, J=1.9 \mathrm{~Hz}, 1 \mathrm{H}), 5.02(\mathrm{~s}, 1 \mathrm{H}), 4.36-4.25(\mathrm{~m}, 3 \mathrm{H}), 2.43(\mathrm{~s}, 3 \mathrm{H}), 1.45(\mathrm{~d}, J=6.4 \mathrm{~Hz}, 3 \mathrm{H})$, 1.28 (s, 12H). ${ }^{13} \mathrm{C}-\mathrm{NMR}\left(75 \mathrm{MHz}, \mathrm{CDCl}_{3}\right) \delta 154.2,148.9,143.4,137.8,129.6,127.5,107.4,83.6$, $59.8,52.6,24.7,23.1,21.5$.

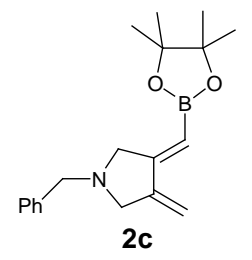

${ }^{1} \mathrm{H}-\mathrm{NMR}\left(300 \mathrm{MHz}, \mathrm{CDCl}_{3}\right) \delta$ 7.38-7.20 (m, 5H), $5.73(\mathrm{~s}, 1 \mathrm{H}), 5.52(\mathrm{~s}, 1 \mathrm{H}), 4.98(\mathrm{~s}, 1 \mathrm{H}), 3.72(\mathrm{~s}, 2 \mathrm{H})$, $3.35(\mathrm{~s}, 2 \mathrm{H}), 3.28(\mathrm{~d}, J=4.2 \mathrm{~Hz}, 2 \mathrm{H}), 1.28(\mathrm{~s}, 12 \mathrm{H})$.

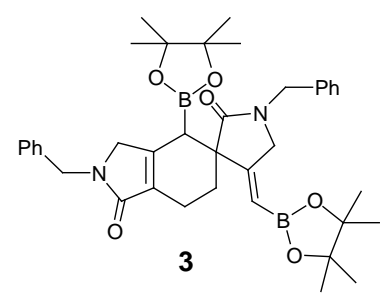

The dimerisation product was the only isolated compound. $35 \%$ from 1d. ${ }^{1} \mathrm{H}-\mathrm{NMR}\left(300 \mathrm{MHz}, \mathrm{CDCl}_{3}\right)$ $\delta$ 7.49-7.28 (m, 10H), $5.19(\mathrm{t}, J=2.2 \mathrm{~Hz}, 1 \mathrm{H}), 5.05(\mathrm{~d}, J=15.1 \mathrm{~Hz}, 1 \mathrm{H}), 4.81(\mathrm{~d}, J=15.0 \mathrm{~Hz}, 1 \mathrm{H})$, $4.38(\mathrm{~d}, J=15.0 \mathrm{~Hz}, 1 \mathrm{H}), 4.28(\mathrm{~d}, J=15.1 \mathrm{~Hz}, 1 \mathrm{H}), 4.27(\mathrm{dd}, J=18.9,3.2 \mathrm{~Hz}, 1 \mathrm{H}), 4.14(\mathrm{dd}, J=16.5$, $2.5 \mathrm{~Hz}, 1 \mathrm{H}), 4.05$ (dd, $J=16.5,2.5 \mathrm{~Hz}, 1 \mathrm{H}), 3.70$ (d, $J=19.0 \mathrm{~Hz}, 1 \mathrm{H}), 2.83-2.80(\mathrm{~m}, 1 \mathrm{H}), 2.58-2.47$ (m, 1H), 2.33-2.24 (m, 1H), $1.90(\mathrm{dt}, J=11.7,5.2 \mathrm{~Hz}, 1 \mathrm{H}), 1.72-1.65(\mathrm{~m}, 1 \mathrm{H}), 1.19(\mathrm{~s}, 6 \mathrm{H}), 1.17$ (s, $6 \mathrm{H}), 1.12(\mathrm{~s}, 6 \mathrm{H}), 1.02(\mathrm{~s}, 6 \mathrm{H})$. 


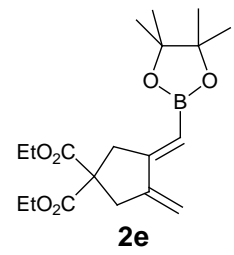

${ }^{1} \mathrm{H}-\mathrm{NMR}\left(300 \mathrm{MHz}, \mathrm{CDCl}_{3}\right) \delta 5.80(\mathrm{t}, J=2.3 \mathrm{~Hz}, 1 \mathrm{H}), 5.53(\mathrm{t}, J=2.3 \mathrm{~Hz}, 1 \mathrm{H}), 5.04(\mathrm{t}, J=1.8 \mathrm{~Hz}$, $1 \mathrm{H}), 4.21$ (q, $J=7.1 \mathrm{~Hz}, 4 \mathrm{H}), 3.34(\mathrm{~m}, 2 \mathrm{H}), 3.05(\mathrm{~m}, 2 \mathrm{H}), 1.29(\mathrm{~s}, 12 \mathrm{H}), 1.25$ (t, $J=7.1 \mathrm{~Hz}, 6 \mathrm{H})$.

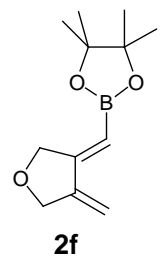

${ }^{1} \mathrm{H}-\mathrm{NMR}\left(300 \mathrm{MHz}, \mathrm{CDCl}_{3}\right) \delta 5.80(\mathrm{t}, J=2.3 \mathrm{~Hz}, 1 \mathrm{H}), 5.57(\mathrm{t}, J=2.3 \mathrm{~Hz}, 1 \mathrm{H}), 5.05(\mathrm{~s}, 1 \mathrm{H}), 4.69(\mathrm{~d}, J$ $=2.3 \mathrm{~Hz}, 2 \mathrm{H}), 4.47(\mathrm{t}, J=2.3 \mathrm{~Hz}, 2 \mathrm{H}), 1.27(\mathrm{~s}, 12 \mathrm{H})$.

\section{IV-Cycloisomerisation/Diels-alder cycloaddition}

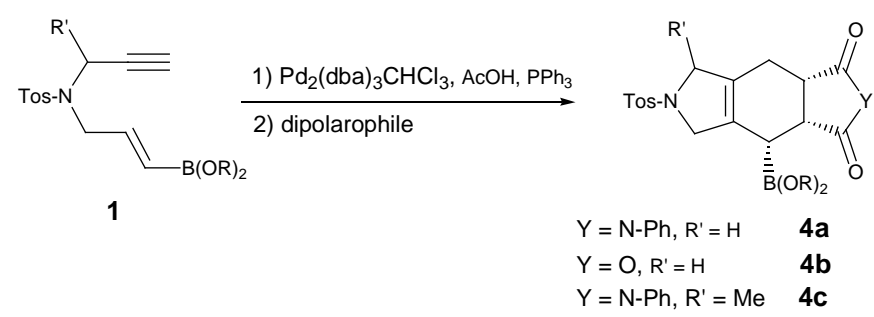

A Schlenk flask with a magnetic stirrer and a rubber septum was charged with tris(dibenzylideneacetone)dipalladium (0) chloroform adduct (5.2 mg, $0.05 \mathrm{mmol}, 2.5 \% \mathrm{~mol}$ ), tri-otolylphosphine $(3.0 \mathrm{mg}, 0.01 \mathrm{mmol}, 5 \% \mathrm{~mol})$, acetic acid $\left(0.6 \mu \mathrm{L}, 10^{-2} \mathrm{mmol}, 5 \% \mathrm{~mol}\right)$ and toluene $(1$ $\mathrm{mL})$. After stirring $15 \mathrm{~min}$ at room temperature, enyne $1(0.2 \mathrm{mmol})$ was added and the mixture was kept $2 \mathrm{~h}$ at room temperature under an argon atmosphere. N-phenylmaleimide $(0.3 \mathrm{mmol}, 1.5 \mathrm{eq})$ or maleic anhydride was then added and the mixture was stirred for $15 \mathrm{~h}$ at room temperature. After removal of volatiles, the residue was purified by washing with ethanol. 
4a (85 mg, 77\%). ${ }^{1} \mathrm{H}-\mathrm{NMR}\left(300 \mathrm{MHz}, \mathrm{CDCl}_{3}\right) \delta 7.75(\mathrm{~d}, J=8.2 \mathrm{~Hz}, 2 \mathrm{H}), 7.48-7.22(\mathrm{~m}, 7 \mathrm{H}), 4.30$ $4.10(\mathrm{~m}, 4 \mathrm{H}), 3.36(\mathrm{dd}, J=9.8,7.6 \mathrm{~Hz}, 1 \mathrm{H}), 3.21(\mathrm{dt}, J=9.8,9.3 \mathrm{~Hz}, 1 \mathrm{H}), 2.63-2.32(\mathrm{~m}, 3 \mathrm{H}), 2.42(\mathrm{~s}$, $3 \mathrm{H}), 1.10(\mathrm{~s}, 12 \mathrm{H}) .{ }^{13} \mathrm{C}-\mathrm{NMR}\left(75 \mathrm{MHz}, \mathrm{CDCl}_{3}\right) \delta 178.5,178.4,143.4,134.1,132.0,131.1,129.8$, $129.0,128.9,128.5,127.5,126.3,84.3,56.6,56.5,40.8,38.7,24.6,24.2,22.2,21.5$. MS (LSIMS) calcd. For $\mathrm{C}_{29} \mathrm{H}_{34} \mathrm{~N}_{2} \mathrm{O}_{6}{ }^{11} \mathrm{BS}[\mathrm{M}+\mathrm{H}]^{+}$: 549.2230, found : 549.2236 .

4b (78 mg, 83\%). ${ }^{1} \mathrm{H}-\mathrm{NMR}\left(300 \mathrm{MHz}, \mathrm{CD}_{3} \mathrm{COCD}_{3}\right) \delta 7.72(\mathrm{~d}, J=8.2 \mathrm{~Hz}, 2 \mathrm{H}), 7.34(\mathrm{~d}, J=8.2 \mathrm{~Hz}$, 2H), 4.18-4.08 (m, 4H), 3.42-3.26 (m, 2H), 2.55-2.33 (m, 3H), 2.43 (s, 3H), 1.14 (s, 6H), 1.12 (s, 6H).

${ }^{13} \mathrm{C}-\mathrm{NMR}\left(75 \mathrm{MHz}, \mathrm{CD}_{3} \mathrm{COCD}_{3}\right) \delta$ 174.4, 174.2, 143.6, 134.1, 129.8, 128.7, 127.5, 84.3, 56.6, 56.3, 40.8, 38.4, 24.0, 23.7, 21.0, 20.5. MS (EI) calcd. For $\mathrm{C}_{22} \mathrm{H}_{28} \mathrm{NO}_{6}{ }^{11} \mathrm{BS}[\mathrm{M}-\mathrm{CO}]^{+}: 445.1730$, found : 445.1702 .

4c. ${ }^{1} \mathrm{H}-\mathrm{NMR}\left(300 \mathrm{MHz}, \mathrm{CDCl}_{3}\right) \delta 7.75(\mathrm{~d}, J=8.2 \mathrm{~Hz}, 2 \mathrm{H}), 7.50-7.27(\mathrm{~m}, 7 \mathrm{H}), 4.43-4.11(\mathrm{~m}, 3 \mathrm{H}), 3.32$ $(\mathrm{dd}, J=9.8,7.2 \mathrm{~Hz}, 1 \mathrm{H}), 3.16(\mathrm{dt}, J=9.7,7.6 \mathrm{~Hz}, 1 \mathrm{H}), 2.46-2.41(\mathrm{~m}, 2 \mathrm{H}), 2.45(\mathrm{~s}, 3 \mathrm{H}), 2.29(\mathrm{~d}, J=$ $6.7 \mathrm{~Hz}, 1 \mathrm{H}), 1.36(\mathrm{~d}, J=6.3 \mathrm{~Hz}, 3 \mathrm{H}), 1.21(\mathrm{~s}, 12 \mathrm{H})$.

\section{V- General procedure for palladium-catalyzed cycloisomerisation /Diels-Alder cycloaddition/allylboration sequence}

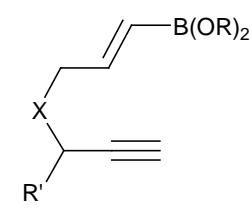

1

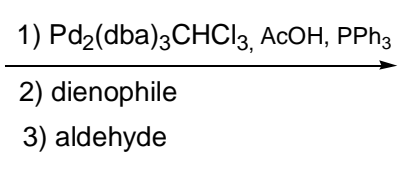

3) aldehyde

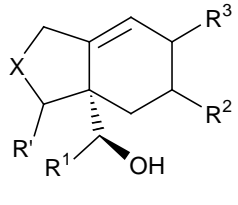

5-15

In a Schlenk flask tris(dibenzylideneacetone)dipalladium(0)-chloroform adduct $(5.2 \mathrm{mg}, 0.05 \mathrm{mmol}$, $2 \% \mathrm{~mol})$ tri-o-tolylphosphine $\left(3 \mathrm{mg}, 0.01 \mathrm{mmol}\right.$, acetic acid $\left(0.6 \mu \mathrm{L}, 10^{-2} \mathrm{mmol}, 5 \% \mathrm{~mol}\right)$ and toluene $(1 \mathrm{~mL})$ were stirred for $15 \mathrm{~min}$. Enyne $1(0.2 \mathrm{mmol})$ was added and the mixture was reacted $2 \mathrm{~h}$ at room temperature under an argon atmosphere $\left(18 \mathrm{~h}\right.$ for $1 \mathrm{c}$ and $2 \mathrm{~h}$ at $50^{\circ} \mathrm{C}$ for $\left.\mathbf{1 b}\right)$. Then dienophile $(0.3$ mmol, $1.5 \mathrm{eq})$ and aldehyde $(0.4 \mathrm{mmol}, 2 \mathrm{eq})$ were successively added and the mixture was stirred for $15 \mathrm{~h}$ at $60^{\circ} \mathrm{C}$. For $\mathbf{4 b}$, toluene was first evaporated before performing the allylboration in THF. After removal of volatiles, THF $(2 \mathrm{~mL})$ and saturated $\mathrm{Na}_{2} \mathrm{CO}_{3}(2 \mathrm{~mL})$ were added and the mixture was stirred for $15 \mathrm{~h}$. Ethyl acetate $(10 \mathrm{~mL})$ and water $(2 \mathrm{~mL})$ were added and the aqueous phase was extracted with ethyl acetate $(2 \times 10 \mathrm{~mL})$. The combined organic phases were washed with $\mathrm{H}_{2} \mathrm{O}(5 \mathrm{~mL})$ 
and brine $(5 \mathrm{~mL})$, dried over $\mathrm{MgSO}_{4}$ and evaporated under vacuo. The residue was purified by column chromatography on silica gel (cyclohexane/ethyl acetate: $1 / 4$ to $1 / 1$ ).

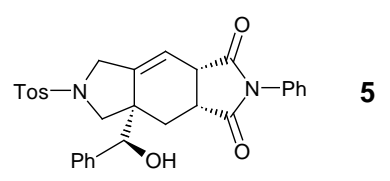

5 (75 mg, 71\%). ${ }^{1} \mathrm{H}-\mathrm{NMR}\left(300 \mathrm{MHz}, \mathrm{CDCl}_{3}\right) \delta 7.66(\mathrm{~d}, J=8.2 \mathrm{~Hz}, 2 \mathrm{H}), 7.46-7.28(\mathrm{~m}, 12 \mathrm{H}), 6.03$ (d, $J=6.1 \mathrm{~Hz}, 1 \mathrm{H}), 4.70(\mathrm{~d}, J=3.2 \mathrm{~Hz}, 1 \mathrm{H}), 4.05(\mathrm{dt}, J=14.1,2.1 \mathrm{~Hz}, 1 \mathrm{H}), 3.79-3.66(\mathrm{~m}, 3 \mathrm{H}), 3.25(\mathrm{t}, J$ $=9.3 \mathrm{~Hz}, 1 \mathrm{H}), 2.93(\mathrm{~d}, J=14.7 \mathrm{~Hz}, 1 \mathrm{H}), 2.46(\mathrm{~s}, 3 \mathrm{H}), 2.39(\mathrm{~d}, J=9.7 \mathrm{~Hz}, 1 \mathrm{H}), 2.19(\mathrm{~d}, J=3.2 \mathrm{~Hz}$, $1 \mathrm{H}), 1.27$ (dd, $J=15.0,9.5 \mathrm{~Hz}, 1 \mathrm{H} .{ }^{13} \mathrm{C}-\mathrm{NMR}$ (75 MHz, DMSO-D6) $\delta 183.8,181.5,148.9,145.5$, 144.9, 138.0, 137.6, 135.1, 133.9, 133.3, 132.9, 132.8, 132.7, 132.6, 132.3, 122.2, 78.1, 61.7, 55.5, 53.2, 42.3, 29.8, 26.2. MS (LSIMS) calcd. For $\mathrm{C}_{30} \mathrm{H}_{29} \mathrm{~N}_{2} \mathrm{O}_{5} \mathrm{~S}[\mathrm{M}+\mathrm{H}]^{+}:$529.1797, found: 529.1776 .

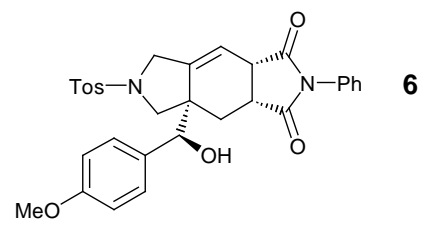

6 (82 mg, 74\%). ${ }^{1} \mathrm{H}-\mathrm{NMR}\left(300 \mathrm{MHz}, \mathrm{CDCl}_{3}\right) \delta 7.68(\mathrm{~d}, J=8.2 \mathrm{~Hz}, 2 \mathrm{H}), 7.50-7.23(\mathrm{~m}, 9 \mathrm{H}), 6.88(\mathrm{~d}, J$ $=8.2 \mathrm{~Hz}, 2 \mathrm{H}), 6.04(\mathrm{~d}, J=6.0 \mathrm{~Hz}, 1 \mathrm{H}), 4.70(\mathrm{~d}, J=3.2 \mathrm{~Hz}, 1 \mathrm{H}), 4.09(\mathrm{dt}, J=13.9,1.9 \mathrm{~Hz}, 1 \mathrm{H}), 3.82$ (s, 3H), 3.78-3.70 (m, 2H), $3.64(\mathrm{~d}, J=9.6 \mathrm{~Hz}, 1 \mathrm{H}), 3.25(\mathrm{t}, J=9.3 \mathrm{~Hz}, 1 \mathrm{H}), 2.91$ (d, $J=14.9 \mathrm{~Hz}, 1 \mathrm{H})$, $2.46(\mathrm{~s}, 3 \mathrm{H}), 2.38(\mathrm{~d}, J=9.7 \mathrm{~Hz}, 1 \mathrm{H}), 2.02(\mathrm{~d}, J=3 \mathrm{~Hz}, 1 \mathrm{H}), 1.28(\mathrm{dd}, J=15.2,9.6 \mathrm{~Hz}, 1 \mathrm{H}) .{ }^{13} \mathrm{C}-$ NMR (75 MHz, DMSO-D6) $\delta$ 179.0, 176.8, 159.0, 144.2, 140.3, 133.6, 133.2, 132.9, 130.4, 129.1, 128.5, 128.0, 127.6, 117.3, 113.5, 72.9, 57.0, 55.5, 50.8, 48.6, 37.5, 25.2, 21.5. MS (ESI) calcd. For $\mathrm{C}_{31} \mathrm{H}_{30} \mathrm{~N}_{2} \mathrm{O}_{6} \mathrm{NaS}[\mathrm{M}+\mathrm{Na}]^{+}: 581.1722$, found: 581.1731 .

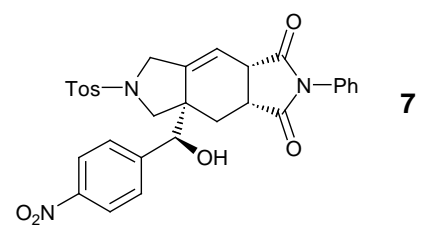

$7(89 \mathrm{mg}, 78 \%) .{ }^{1} \mathrm{H}-\mathrm{NMR}\left(300 \mathrm{MHz}, \mathrm{CDCl}_{3}\right) \delta 8.18(\mathrm{~d}, J=8.7 \mathrm{~Hz}, 2 \mathrm{H}), 7.63(\mathrm{~d}, J=8.7 \mathrm{~Hz}, 2 \mathrm{H}), 7.60$ $(\mathrm{d}, J=8.7 \mathrm{~Hz}, 2 \mathrm{H}), 7.50-7.25(\mathrm{~m}, 7 \mathrm{H}), 6.1(\mathrm{~d}, J=7.3 \mathrm{~Hz}, 1 \mathrm{H}), 4.79(\mathrm{~d}, J=3.6 \mathrm{~Hz}, 1 \mathrm{H}), 3.98(\mathrm{~d}, J=$ $13.8 \mathrm{~Hz}, 1 \mathrm{H}), 3.85-3.73(\mathrm{~m}, 2 \mathrm{H}), 3.65(\mathrm{~d}, J=9.9 \mathrm{~Hz}, 1 \mathrm{H}), 3.30(\mathrm{t}, J=9.1 \mathrm{~Hz}, 1 \mathrm{H}), 2.86(\mathrm{~d}, J=15.1$ 
$\mathrm{Hz}, 1 \mathrm{H}), 2.50-2.38(\mathrm{~m}, 5 \mathrm{H}), 1.37$ (dd, $J=15.0,9.2 \mathrm{~Hz}, 1 \mathrm{H}) .{ }^{13} \mathrm{C}-\mathrm{NMR}$ (75 MHz, DMSO-D6) $\delta 179.1$, $176.7,149.7,147.2$, 144.3, 139.7, 133.1, 132.6, 130.4, 129.2, 129.0, 128.6, 128.0, 127.5, 127.4, 123.2, 118.2, 72.9, 56.4, 50.4, 50.7, 48.7, 25.6, 21.5. MS (ESI) calcd. For $\mathrm{C}_{30} \mathrm{H}_{27} \mathrm{~N}_{3} \mathrm{O}_{7} \mathrm{NaS}[\mathrm{M}+\mathrm{Na}]^{+}$: 596.1467, found: 596.1457.

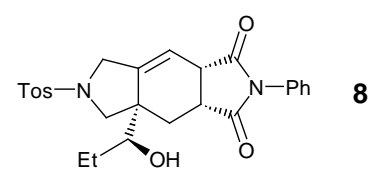

$8(65 \mathrm{mg}, 69 \%) .{ }^{1} \mathrm{H}-\mathrm{NMR}\left(300 \mathrm{MHz}, \mathrm{CDCl}_{3}\right) \delta 7.72(\mathrm{~d}, J=8.1 \mathrm{~Hz}, 2 \mathrm{H}), 7.50-7.18(\mathrm{~m}, 7 \mathrm{H}), 5.98(\mathrm{~d}, J$ $=5.6 \mathrm{~Hz}, 1 \mathrm{H}), 4.07(\mathrm{~d}, J=14 \mathrm{~Hz}, 1 \mathrm{H}), 3.89(\mathrm{~d}, J=14 \mathrm{~Hz}, 1 \mathrm{H}), 3.72(\mathrm{dd}, J=8.1,6.9 \mathrm{~Hz}, 1 \mathrm{H}), 3.64(\mathrm{~d}$, $J=10.0 \mathrm{~Hz}, 1 \mathrm{H}), 3.18(\mathrm{t}, J=8.8 \mathrm{~Hz}, 1 \mathrm{H}), 2.95(\mathrm{~d}, J=8.5 \mathrm{~Hz}, 1 \mathrm{H}), 2.84(\mathrm{~d}, J=14.3 \mathrm{~Hz}, 1 \mathrm{H}), 2.76(\mathrm{~d}$, $J=10.0 \mathrm{~Hz}, 1 \mathrm{H}), 2.45(\mathrm{~s}, 3 \mathrm{H}), 1.85(\mathrm{~d}, J=3.4 \mathrm{~Hz}, 1 \mathrm{H}), 1.60-1.20(\mathrm{~m}, 3 \mathrm{H}), 0.86(\mathrm{t}, J=7.2 \mathrm{~Hz}, 3 \mathrm{H})$. ${ }^{13} \mathrm{C}-\mathrm{NMR}$ (75 MHz, DMSO-D6) $\delta$ 178.4, 176.7, 144.0, 140.6, 133.4, 133.3, 130.4, 129.0, 128.4, 1278, 127.6, 117.0, 72.3, 58.0, 51.0, 48.7, 37.4, 31.2, 25.6, 24.2, 21.5, 12.4. MS (ESI) calcd. For $\mathrm{C}_{26} \mathrm{H}_{28} \mathrm{~N}_{2} \mathrm{O}_{5} \mathrm{NaS}[\mathrm{M}+\mathrm{Na}]^{+}: 503.1616$, found: 503.1617 .

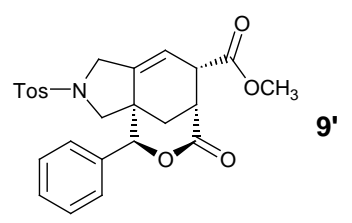

9 was purified as its methyl ester. The residue was dissolved in THF ( $5 \mathrm{~mL})$. After addition of $\mathrm{MeOH}$ $(1 \mathrm{~mL})$ and trimethylsilyldiazomethane (2M in diethylether, $500 \mu 1,4 \mathrm{eq})$, the reaction mixture was stirred $15 \mathrm{~h}$ at room temperature. After evaporation, the residue was purified by column chromatography on silica gel (cyclohexane/ethyl acetate: $2 / 1$ ).

9' (66 mg, $71 \%$ ), mp: $145-148^{\circ} \mathrm{C}$ (diisopropylether). ${ }^{1} \mathrm{H}-\mathrm{NMR}\left(300 \mathrm{MHz}, \mathrm{CDCl}_{3}\right) \delta 7.70(\mathrm{~d}, J=8.2$ $\mathrm{Hz}, 2 \mathrm{H}), 7.47-7.28(\mathrm{~m}, 7 \mathrm{H}), 5.88(\mathrm{t}, J=2.05 \mathrm{~Hz}, 1 \mathrm{H}), 5.50(\mathrm{~s}, 1 \mathrm{H}), 4.43(\mathrm{dt}, J=14.3,2.6 \mathrm{~Hz}, 1 \mathrm{H})$, $3.77(\mathrm{dd}, J=14.3,1.8 \mathrm{~Hz}, 1 \mathrm{H}), 3.72(\mathrm{~s}, 3 \mathrm{H}), 3.58(\mathrm{~d}, J=4.05 \mathrm{~Hz}, 1 \mathrm{H}), 3.53-3.49(\mathrm{~m}, 2 \mathrm{H}), 3.36(\mathrm{~d}, J=$ $10.0 \mathrm{~Hz}, 1 \mathrm{H}), 3.31(\mathrm{dd}, J=13.5,4.3 \mathrm{~Hz}, 1 \mathrm{H}), 2.48(\mathrm{~s}, 3 \mathrm{H}), 1.38(\mathrm{~d}, J=13.5 \mathrm{~Hz}, 1 \mathrm{H}) .{ }^{13} \mathrm{C}-\mathrm{NMR}(75$ $\left.\mathrm{MHz}, \mathrm{CDCl}_{3}\right) \delta 171.8,170.75,144.25,140.5,135.7,132.3,130.0,128.9,128.6,127.8,127.2,118.2$, 84.5, 55.0, 52.6, 49.6, 45.7, 43.9, 38.2, 21.95, 21.6. MS (EI) calcd. For $\mathrm{C}_{25} \mathrm{H}_{25} \mathrm{NO}_{6} \mathrm{~S}[\mathrm{M}+\mathrm{H}]^{+}$: 467.14026, found: 467.1377 . 


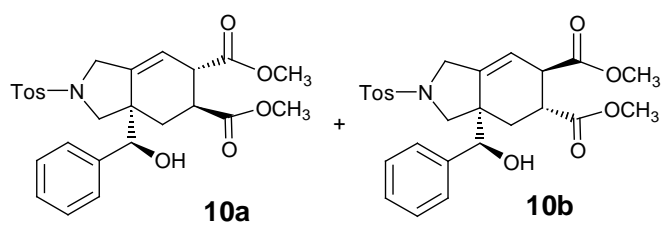

10 (46 mg, $47 \%$ ), mixture of 2 isomers (60/40). Major isomer: ${ }^{1} \mathrm{H}-\mathrm{NMR}\left(300 \mathrm{MHz}, \mathrm{CDCl}_{3}\right) \delta 7.64(\mathrm{~d}$, $J=8.2 \mathrm{~Hz}, 2 \mathrm{H}), 7.38-7.26(\mathrm{~m}, 7 \mathrm{H}), 5.77(\mathrm{~d}, J=2.0 \mathrm{~Hz}, 1 \mathrm{H}), 4.87(\mathrm{~s}, 1 \mathrm{H}), 4.00(\mathrm{dd}, J=5.5,2.1 \mathrm{~Hz}$, $1 \mathrm{H}), 3.85$ (d, $J=9.8 \mathrm{~Hz}, 1 \mathrm{H}), 3.79-3.48(\mathrm{~m}, 1 \mathrm{H}), 3.75(\mathrm{~s}, 3 \mathrm{H}), 3.71(\mathrm{~s}, 3 \mathrm{H}), 2.91-2.88(\mathrm{~m}, 1 \mathrm{H}), 2.78$ (dt, $J=8.6,2.0 \mathrm{~Hz}, 1 \mathrm{H}), 2.64$ (d, $J=9.7 \mathrm{~Hz}, 1 \mathrm{H}), 2.48$ (d, $J=4.2 \mathrm{~Hz}, 1 \mathrm{H}), 2.45$ (s, 3H), 2.15 (s, br, 1H), $1.40(\mathrm{dd}, J=14.1,6.6 \mathrm{~Hz}, 1 \mathrm{H}) ;{ }^{13} \mathrm{C}-\mathrm{NMR}\left(75 \mathrm{MHz}, \mathrm{CDCl}_{3}\right) \delta 174.8,172.8,143.5,139.7,138.7$, 133.5, 129.65, 128.1, 127.3 (2C), 119.9, 74.6, 55.5, 52.4, 52.2, 50.6, 49.4, 43.8, 38.5, 31.3, 21.4 (2C). Minor isomer (characteristic signals): ${ }^{1} \mathrm{H}-\mathrm{NMR}\left(300 \mathrm{MHz}, \mathrm{CDCl}_{3}\right) \delta 7.72(\mathrm{~d}, J=8.3 \mathrm{~Hz}, 2 \mathrm{H}), 5.83(\mathrm{~d}$, $J=1.7 \mathrm{~Hz}, 1 \mathrm{H}), 4.71(\mathrm{~s}, 1 \mathrm{H}), 3.73(\mathrm{~s}, 3 \mathrm{H}), 3.68(\mathrm{~s}, 3 \mathrm{H}), 2.56(\mathrm{~d}, J=8.9 \mathrm{~Hz}, 1 \mathrm{H}), 2.39$ (d, $J=3.8 \mathrm{~Hz}$, $1 \mathrm{H}) ;{ }^{13} \mathrm{C}-\mathrm{NMR}\left(75 \mathrm{MHz}, \mathrm{CDCl}_{3}\right) \delta 175.2,173.3,143.6,139.9,139.4,133.4,129.7,128.0,127.9$, $127.1,121.1,73.5,56.4,52.4,52.25,50.4,49.9,42.5,40.6,27.5,22.7$. MS (EI) calcd. For $\mathrm{C}_{25} \mathrm{H}_{26} \mathrm{NO}_{6} \mathrm{~S}\left[\mathrm{M}-\mathrm{OCH}_{3}\right]^{+}: 468.1481$, found : 468.1484 .

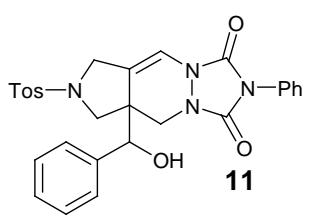

After purification by chromatography on silica gel, three compounds were isolated: 11, the addition product to 4-phenyl triazolidinedione and another unidentified by-product.

11 (25 mg, 23\%). ${ }^{1} \mathrm{H}-\mathrm{NMR}\left(300 \mathrm{MHz}, \mathrm{CDCl}_{3} / \mathrm{DMSO}-\mathrm{D} 6\right) \delta 7.33(\mathrm{~d}, J=8.2 \mathrm{~Hz}, 2 \mathrm{H}), 7.28-7.16$ (m, $6 \mathrm{H}), 7.10(\mathrm{~d}, J=8.1 \mathrm{~Hz}, 2 \mathrm{H}), 6.99-6.85(\mathrm{~m}, 4 \mathrm{H}), 6.69(\mathrm{~s}, 1 \mathrm{H}), 4.59(\mathrm{~d}, J=11.7 \mathrm{~Hz}, 1 \mathrm{H}), 4.54(\mathrm{~s}, 1 \mathrm{H})$, $3.68(\mathrm{~d}, J=10.4 \mathrm{~Hz}, 1 \mathrm{H}), 3.48(\mathrm{~d}, J=12.7 \mathrm{~Hz}, 1 \mathrm{H}), 2.78(\mathrm{~d}, J=4.2 \mathrm{~Hz}, 1 \mathrm{H}), 2.74(\mathrm{~d}, J=2.9 \mathrm{~Hz}, 1 \mathrm{H})$, 2.67 (s, br, 1H), 2.55 (dd, $J=12.2,1.8 \mathrm{~Hz}, 1 \mathrm{H}), 2.25$ (s, 3H). ${ }^{13} \mathrm{C}-\mathrm{NMR}$ (75 MHz, CDCl $/ \mathrm{DMSO}^{-\mathrm{D} 6)}$ $\delta 148.7,144.5,143.0,139.6,132.4,130.35,129.1,128.4,127.6,127.1,126.9,126.8,126.3,125.0$, 117.3, 111.9, 72.7, 49.9, 49.6, 49.0, 45.5, 20.9. MS (EI) calcd. For $\mathrm{C}_{21} \mathrm{H}_{20} \mathrm{~N}_{4} \mathrm{O}_{4} \mathrm{~S}$ [M-PhCHO] ${ }^{+}$: 424.12053, found: 424.1191 . 


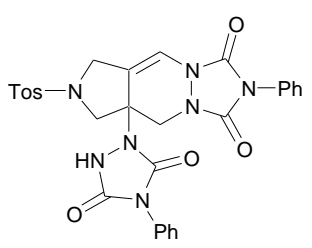

(10 mg, 8\%). ${ }^{1} \mathrm{H}-\mathrm{NMR}\left(300 \mathrm{MHz}, \mathrm{CDCl}_{3}\right) \delta 7.60(\mathrm{~d}, J=7.9 \mathrm{~Hz}, 2 \mathrm{H}), 7.33-7.03(\mathrm{~m}, 12 \mathrm{H}), 6.84(\mathrm{~s}$, 1H), $4.78(\mathrm{~d}, J=13.1 \mathrm{~Hz}, 1 \mathrm{H}), 4.35(\mathrm{dd}, J=15.35,14.1 \mathrm{~Hz}, 2 \mathrm{H}), 3.79(\mathrm{~d}, J=13.7 \mathrm{~Hz}, 1 \mathrm{H}), 2.84(\mathrm{~d}, J$ $=10.3 \mathrm{~Hz}, 1 \mathrm{H}), 2.57(\mathrm{~d}, J=11.4 \mathrm{~Hz}, 1 \mathrm{H}), 2.19$ (s, 3H). ${ }^{13} \mathrm{C}-\mathrm{NMR}\left(75 \mathrm{MHz}, \mathrm{CD}_{3} \mathrm{OD}\right) \delta 156.5,152.8$, $152.45,147.4,145.4,135.3,135.1,132.5,130.9,130.1,129.6,129.5,128.8,127.7,127.5,127.0$, 116.0, 115.8, 63.3, 56.6, 50.6, 48.0, 21.7. MS (ESI) calcd. For $\mathrm{C}_{29} \mathrm{H}_{25} \mathrm{~N}_{7} \mathrm{O}_{6} \mathrm{NaS}[\mathrm{M}+\mathrm{Na}]^{+}: 622.1485$, found: 622.1464 .

Unidentified product $(21 \mathrm{mg}) .{ }^{1} \mathrm{H}-\mathrm{NMR}\left(300 \mathrm{MHz}, \mathrm{CDCl}_{3} / \mathrm{DMSO}-\mathrm{D} 6\right) \delta 7.60(\mathrm{~d}, J=8.1 \mathrm{~Hz}, 2 \mathrm{H})$, 7.35-7.23 (m, 7H), $5.66(\mathrm{~s}, 1 \mathrm{H}), 4.25-3.99(\mathrm{~m}, 5 \mathrm{H}), 3.78(\mathrm{~d}, J=15.3 \mathrm{~Hz}, 1 \mathrm{H}), 2.46(\mathrm{~s}, 1 \mathrm{H}), 2.32$ (s, $3 \mathrm{H})$.

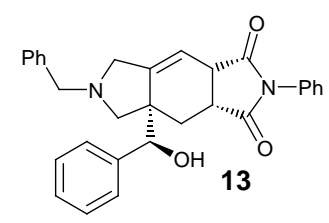

13 (51 mg, 52\%). Mp 178-180 $\mathrm{C}$ (diisopropylether). ${ }^{1} \mathrm{H}-\mathrm{NMR}\left(300 \mathrm{MHz}, \mathrm{CDCl}_{3}\right) \delta$ 7.46-7.26 (m, $15 \mathrm{H}), 5.99(\mathrm{~d}, J=6.2 \mathrm{~Hz}, 1 \mathrm{H}), 4.93(\mathrm{~s}, 1 \mathrm{H}), 3.80(\mathrm{ddt}, J=9.5,6.3,1.75 \mathrm{~Hz}, 1 \mathrm{H}), 3.69(\mathrm{~d}, J=14.1 \mathrm{~Hz}$, $1 \mathrm{H}), 3.62(\mathrm{~d}, J=12.9 \mathrm{~Hz}, 1 \mathrm{H}), 3.57$ (d, $J=12.8 \mathrm{~Hz}, 1 \mathrm{H}), 3.30$ (t, $J=9.4 \mathrm{~Hz}, 1 \mathrm{H}), 3.09$ (d, $J=14.1 \mathrm{~Hz}$, $1 \mathrm{H}), 2.90(\mathrm{~s}, 1 \mathrm{H}), 2.89$ (d, $J=24.8 \mathrm{~Hz}, 1 \mathrm{H}), 2.06$ (s, br, 1H), 1.99 (d, $J=9.3 \mathrm{~Hz}, 1 \mathrm{H}), 1.41(\mathrm{dd}, J=$ 14.9, $9.9 \mathrm{~Hz}, 1 \mathrm{H}) .{ }^{13} \mathrm{C}-\mathrm{NMR}\left(75 \mathrm{MHz}, \mathrm{CDCl}_{3}\right) \delta 179.5,177.1,145.6,140.6,138.7,132.35,129.1$, $128.8,128.4,128.2,128.0,127.8,127.7,127.2,126.4,114.0,75.7,63.7,59.9,56.7,48.95,40.5,38.24$ 25.4. MS (EI) calcd. For $\mathrm{C}_{30} \mathrm{H}_{28} \mathrm{~N}_{2} \mathrm{O}_{3}[\mathrm{M}+\mathrm{H}]^{+}$: 464.20999, found: 464.2116.

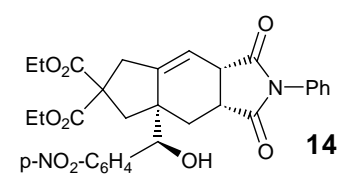

14 (47 mg, 42\%). ${ }^{1} \mathrm{H}-\mathrm{NMR}\left(300 \mathrm{MHz}, \mathrm{CDCl}_{3}\right) \delta 8.21(\mathrm{~d}, J=8.7 \mathrm{~Hz}, 2 \mathrm{H}), 7.59(\mathrm{~d}, J=8.7 \mathrm{~Hz}, 2 \mathrm{H})$, 7.52-7.33 (m, 3H), 7.29-7.22 (m, 2H), 6.04 (dd, $J=6.1,1.9 \mathrm{~Hz}, 1 \mathrm{H}), 4.54$ (d, $J=4 \mathrm{~Hz}, 1 \mathrm{H}), 4.23-4.10$ (m, 4H), 3.90-3.83 (m, 1H), $3.32(\mathrm{t}, J=8.6 \mathrm{~Hz}, 1 \mathrm{H}), 3.05(\mathrm{~d}, J=14.8 \mathrm{~Hz}, 1 \mathrm{H}), 2.80(\mathrm{~d}, J=15.4 \mathrm{~Hz}$, 
1H), 2.58-2.52 (m, 1H), 2.50 (d, $J=8.6 \mathrm{~Hz}, 1 \mathrm{H}), 2.45$ (d, $J=15.4 \mathrm{~Hz}, 1 \mathrm{H}), 1.71(\mathrm{dd}, J=14.8,8.6 \mathrm{~Hz}$, $1 \mathrm{H}), 1.43(\mathrm{~s}, 1 \mathrm{H}), 1.27-1.20(\mathrm{~m}, 6 \mathrm{H}) .{ }^{13} \mathrm{C}-\mathrm{NMR}\left(75 \mathrm{MHz}, \mathrm{CDCl}_{3}\right) \delta 179.6,176.3,171.7,171.2,147.5$, 147.2, 145.4, 131.9, 129.3, 128.9, 128.7, 126.2, 125.4, 123.2, 116.9, 61.9, 56.7, 48.7, 40.8, 40.5, 37.8, 29.7, 29.5, 14.0. MS (ESI) calcd. For $\mathrm{C}_{30} \mathrm{H}_{30} \mathrm{~N}_{2} \mathrm{O}_{9} \mathrm{Na}[\mathrm{M}+\mathrm{Na}]^{+}:$585.1849, found: 585.1851 .

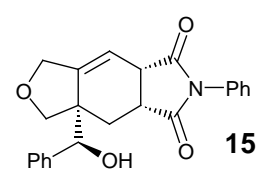

$15(39 \mathrm{mg}, 52 \%) .{ }^{1} \mathrm{H}-\mathrm{NMR}\left(300 \mathrm{MHz}, \mathrm{CDCl}_{3}\right) \delta$ 7.50-7.22 (m, 10H), $6.07(\mathrm{dt}, J=6.1,1.8 \mathrm{~Hz}, 1 \mathrm{H})$, $4.60(\mathrm{~s}, 1 \mathrm{H}), 4.35(\mathrm{~s}, 2 \mathrm{H}), 4.15(\mathrm{~d}, J=9.1 \mathrm{~Hz}, 1 \mathrm{H}), 3.93-3.83(\mathrm{~m}, 1 \mathrm{H}), 3.36(\mathrm{t}, J=9.3 \mathrm{~Hz}, 1 \mathrm{H}), 3.14(\mathrm{~d}, J$ $=10.1 \mathrm{~Hz}, 1 \mathrm{H}), 2.96(\mathrm{~d}, J=14.8 \mathrm{~Hz}, 1 \mathrm{H}), 2.2(\mathrm{~m}, 1 \mathrm{H}), 1.39(\mathrm{dd}, J=14.8,9.3 \mathrm{~Hz}, 1 \mathrm{H}) .{ }^{13} \mathrm{C}-\mathrm{NMR}(75$ $\left.\mathrm{MHz}, \mathrm{CDCl}_{3}\right) \delta 179.2,176.6,144.7,140.0,132.2,129.2,128.5,128.3,128.1,127.8,126.4,114.0$, 76.8, 74.8, 69.8, 49.4, 40.5, 38.3, 24.2. MS (EI) calcd. For $\mathrm{C}_{16} \mathrm{H}_{15} \mathrm{NO}_{3}[\mathrm{M}-\mathrm{PhCHO}]^{+}:$269.1051, found: 269.1067.

\section{VI- Metathesis/Diels-Alder/oxidation sequence}
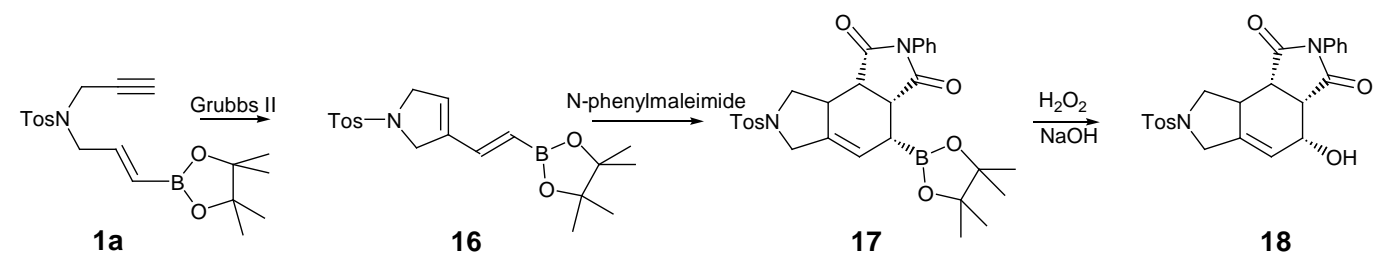

To a solution of the enyne $1 \mathbf{a}(75 \mathrm{mg}, 0.2 \mathrm{mmol})$ in dry $\mathrm{CH}_{2} \mathrm{Cl}_{2}(5 \mathrm{~mL})$ was added Grubbs catalyst $\left(2^{\text {nd }}\right.$ generation) ( $8.5 \mathrm{mg}, 0.01 \mathrm{mmol}, 5 \mathrm{~mol} \%$ ). The solution was stirred for $15 \mathrm{~h}$ at room temperature under an argon atmosphere. The solvent was evaporated and the crude product was used in the next step without further purification. 16 was the only compound detected in the crude ${ }^{1} \mathrm{H}$ NMR. Due to its low stability on silica gel, no attempts were done to purify this diene.

16. ${ }^{1} \mathrm{H}-\mathrm{NMR}\left(300 \mathrm{MHz}, \mathrm{CDCl}_{3}\right) \delta 7.70(\mathrm{~d}, J=8.1 \mathrm{~Hz}, 2 \mathrm{H}), 7.31(\mathrm{~d}, J=8.1 \mathrm{~Hz}, 2 \mathrm{H}), 6.98(\mathrm{~d}, J=18.4$ $\mathrm{Hz}, 1 \mathrm{H}), 5.72$ (s, 1H), 5.33 (d, $J=18.4 \mathrm{~Hz}, 1 \mathrm{H}), 4.19$ (s, 4H), 2.42 (s, 3H), $1.26(\mathrm{~s}, 12 \mathrm{H}) ;{ }^{13} \mathrm{C}-\mathrm{NMR}$ $\left(75 \mathrm{MHz}, \mathrm{CDCl}_{3}\right) \delta 143.6,141.6,138.6,133.9,129.8,127.4,126.4,119.5$ (br), 83.5, 55.4, 53.4, 24.8, 21.5. MS (EI) calcd. for $\mathrm{C}_{19} \mathrm{H}_{26} \mathrm{NO}_{4}{ }^{11} \mathrm{BS}[\mathrm{M}+\mathrm{H}]^{+}: 375.1675$, found: 375.1684 .

The reaction was carried out in the same pot. At the end of the metathesis reaction, the solvent was evaporated and the residue was dissolved in dry toluene. N-phenyl maleimide (52 mg, $0.3 \mathrm{mmol}, 1.5$ 
eq) was added. The solution was stirred for $15 \mathrm{~h}$ at $80^{\circ} \mathrm{C}$. After removing of volatiles, the residue was purified by silica gel chromatography (cyclohexane/ethyl acetate: 6/4) to afford 17.

17 (77 mg, 70\%, two steps). ${ }^{1} \mathrm{H}-\mathrm{NMR}\left(300 \mathrm{MHz}, \mathrm{CDCl}_{3}\right) \delta 7.70(\mathrm{~d}, J=8.2 \mathrm{~Hz}, 2 \mathrm{H}), 7.25-7.16$ (m, $5 \mathrm{H}), 6.96(\mathrm{~d}, J=8.2 \mathrm{~Hz}, 2 \mathrm{H}), 5.98-6.05(\mathrm{~m}, 1 \mathrm{H}), 3.90(\mathrm{~d}, J=13.8 \mathrm{~Hz}, 1 \mathrm{H}), 3.71(\mathrm{dd} J=10.2,8.4 \mathrm{~Hz}$, $1 \mathrm{H}), 3.63-3.48(\mathrm{~m}, 3 \mathrm{H}), 3.39(\mathrm{t}, J=9.1 \mathrm{~Hz}, 1 \mathrm{H}), 2.88-2.78(\mathrm{~m}, 1 \mathrm{H}), 2.43(\mathrm{~s}, 3 \mathrm{H}), 1.80-1.72(\mathrm{~m}, 1 \mathrm{H})$, $1.28(\mathrm{~s}, 12 \mathrm{H}) .{ }^{13} \mathrm{C}-\mathrm{NMR}\left(75 \mathrm{MHz}, \mathrm{CDCl}_{3}\right) \delta 178.1,175.7,143.8,138.7,131.8,131.6,129.6,128.9$, $128.5,128.2,126.3,121.3,84.2,50.8,49.6,43.8,42.1,37.5,24.9,24.8,21.6$. MS (EI) calcd. for $\mathrm{C}_{26} \mathrm{H}_{27} \mathrm{~N}_{2} \mathrm{O}_{5}{ }^{11} \mathrm{BS}\left[\mathrm{M}-\mathrm{CH}_{3}-\mathrm{COCH}_{3}\right]^{+}: 490.1733$, found: 490.1714.

An aqueous solution of $\mathrm{NaOAc}(3 \mathrm{M}, 0.5 \mathrm{~mL}, 1.5 \mathrm{mmol})$ was added dropwise to a precooled solution $\left(0^{\circ} \mathrm{C}\right)$ of the crude 17 (from $0.1 \mathrm{mmol}$ of 1a) in THF $(5 \mathrm{~mL})$ and the temperature maintained below $5^{\circ} \mathrm{C}$. Hydrogen peroxide $(0.5 \mathrm{~mL}, 5.5 \mathrm{mmol})$ was added and the mixture was stirred at $0^{\circ} \mathrm{C}$ for $2 \mathrm{~h}$. Water $(5 \mathrm{~mL})$ was added, and the aqueous layer was extracted with ether $(2 \times 15 \mathrm{~mL})$. The ether layers were washed with aqueous saturated solution of $\mathrm{NaCl}$, dried over $\mathrm{MgSO}_{4}$. After the solvent was removed, the residue was purified by silica gel chromatography (cyclohexane-ethyl acetate: 6/4).

18 (15 mg, 35\%, three steps). ${ }^{1} \mathrm{H}-\mathrm{NMR}\left(300 \mathrm{MHz}, \mathrm{CDCl}_{3}\right) \delta 7.72(\mathrm{~d}, J=8.3 \mathrm{~Hz}, 2 \mathrm{H})$, 7.45-7.28 (m, $5 \mathrm{H}), 7.04$ (d, $J=8.3 \mathrm{~Hz}, 2 \mathrm{H}), 5.94(\mathrm{~s}, 1 \mathrm{H}), 4.41(\mathrm{~m}, 2 \mathrm{H}), 3.86$ (d, $J=19.9 \mathrm{~Hz}, 1 \mathrm{H}), 3.75-3.61(\mathrm{~m}, 3 \mathrm{H})$, $3.51(\mathrm{dd}, J=8.7,5.3 \mathrm{~Hz}, 1 \mathrm{H}), 3.38(\mathrm{t}, J=8.7 \mathrm{~Hz}, 1 \mathrm{H}), 2.81(\mathrm{~m}, 1 \mathrm{H}), 2.06(\mathrm{~s}, 3 \mathrm{H}) .{ }^{13} \mathrm{C}-\mathrm{NMR}(75 \mathrm{MHz}$, $\left.\mathrm{CDCl}_{3}\right) \delta 178.6,174.7,144.0,137.1,130.8,129.7,129.2,129.0,128.2,126.2,126.0,67.8,50.6,49.0$, 44.9, 39.7, 36.9, 24.9, 21.6. MS (EI) calcd. for $\mathrm{C}_{16} \mathrm{H}_{15} \mathrm{~N}_{2} \mathrm{O}_{5}\left[\mathrm{M}-\mathrm{CH}_{3} \mathrm{C}_{6} \mathrm{H}_{5} \mathrm{SO}_{2}\right]^{+}:$283.1082, found: 283.1076 .

\section{VII- Platinum-catalyzed cycloisomerisation of 1a}
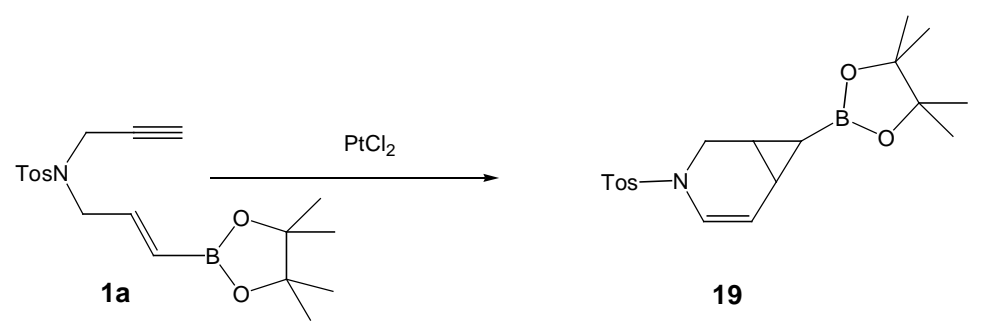

To a solution of boronate $1 \mathrm{a}(150 \mathrm{mg}, 0.54 \mathrm{mmol})$ in dry toluene $(2 \mathrm{~mL})$ was added platinum (II) chloride $(5 \mathrm{mg}, 0.02 \mathrm{mmol})$. The reaction was stirred one hour at $70^{\circ} \mathrm{C}$. The volatiles were evaporated under vacuo. The residue was purified by silica gel chromatography (cyclohexane/ethyl acetate: 1/2). 
$19(126 \mathrm{mg}, 62 \%) .{ }^{1} \mathrm{H}-\mathrm{NMR}\left(300 \mathrm{MHz}, \mathrm{CDCl}_{3}\right) \delta 7.64(\mathrm{~d}, J=8.1 \mathrm{~Hz}, 2 \mathrm{H}), 7.31(\mathrm{~d}, J=8.0 \mathrm{~Hz}, 2 \mathrm{H})$, $6.32(\mathrm{~d}, J=8.0 \mathrm{~Hz}, 1 \mathrm{H}), 5.35(\mathrm{dd}, J=5.35,7.85 \mathrm{~Hz}, 1 \mathrm{H}), 3.86(\mathrm{~d}, J=11.6 \mathrm{~Hz}, 1 \mathrm{H}), 3.10$ (dd, $J=2.7$, $11.5 \mathrm{~Hz}, 1 \mathrm{H}), 2.42(\mathrm{~s}, 3 \mathrm{H}), 1.70(\mathrm{t}, J=6.1 \mathrm{~Hz}, 1 \mathrm{H}), 1.33-1.24(\mathrm{~m}, 2 \mathrm{H}), 1.19(\mathrm{~s}, 12 \mathrm{H}) .{ }^{13} \mathrm{C}-\mathrm{NMR}(75$ $\left.\mathrm{MHz}, \mathrm{CDCl}_{3}\right) \delta 143.7,134.8,129.8,127.0,121.3,110.9,83.5,41.4,24.8,24.6,23.4,21.5,13.3 . \mathrm{MS}$ (EI) calcd. for $\mathrm{C}_{19} \mathrm{H}_{26} \mathrm{NO}_{4}{ }^{11} \mathrm{BS}[\mathrm{M}+\mathrm{H}]^{+}: 375.16756$, found: 375.1684 .

\section{VIII- X-ray structure of 5}

The crystal structure has been deposited at the CambridgeCrystallographic Data Centre and allocated the deposition number CCDC 635610

Summary of Data CCDC 635610

Formula: C30 H28 N2 O5 S1

Unit cell parameters: a 9.0954(3) b 13.7732(4) c 13.8767(4) alpha 61.2960(10) beta 78.0230(10) gamma 77.9740(10) space group P-1

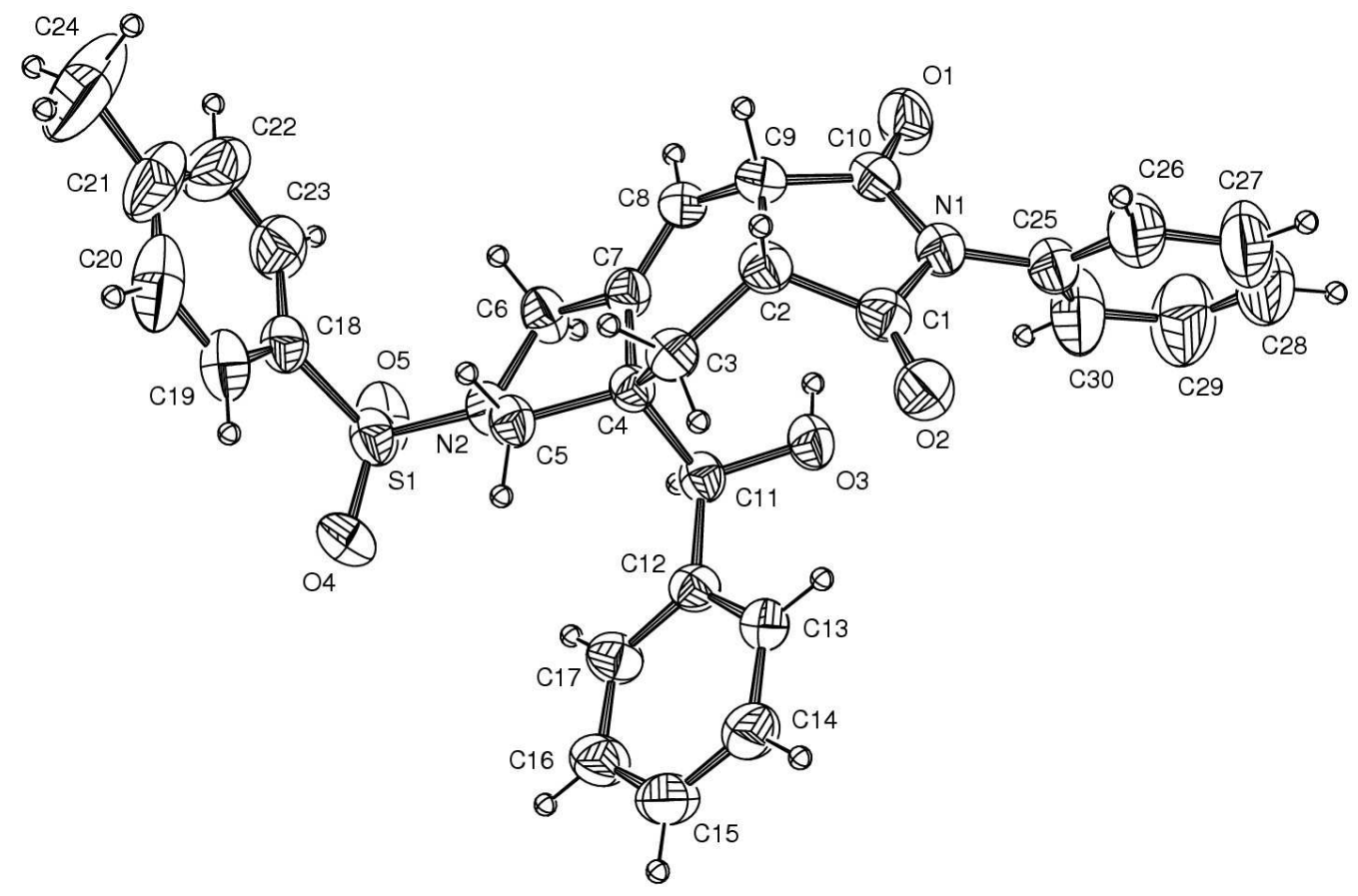




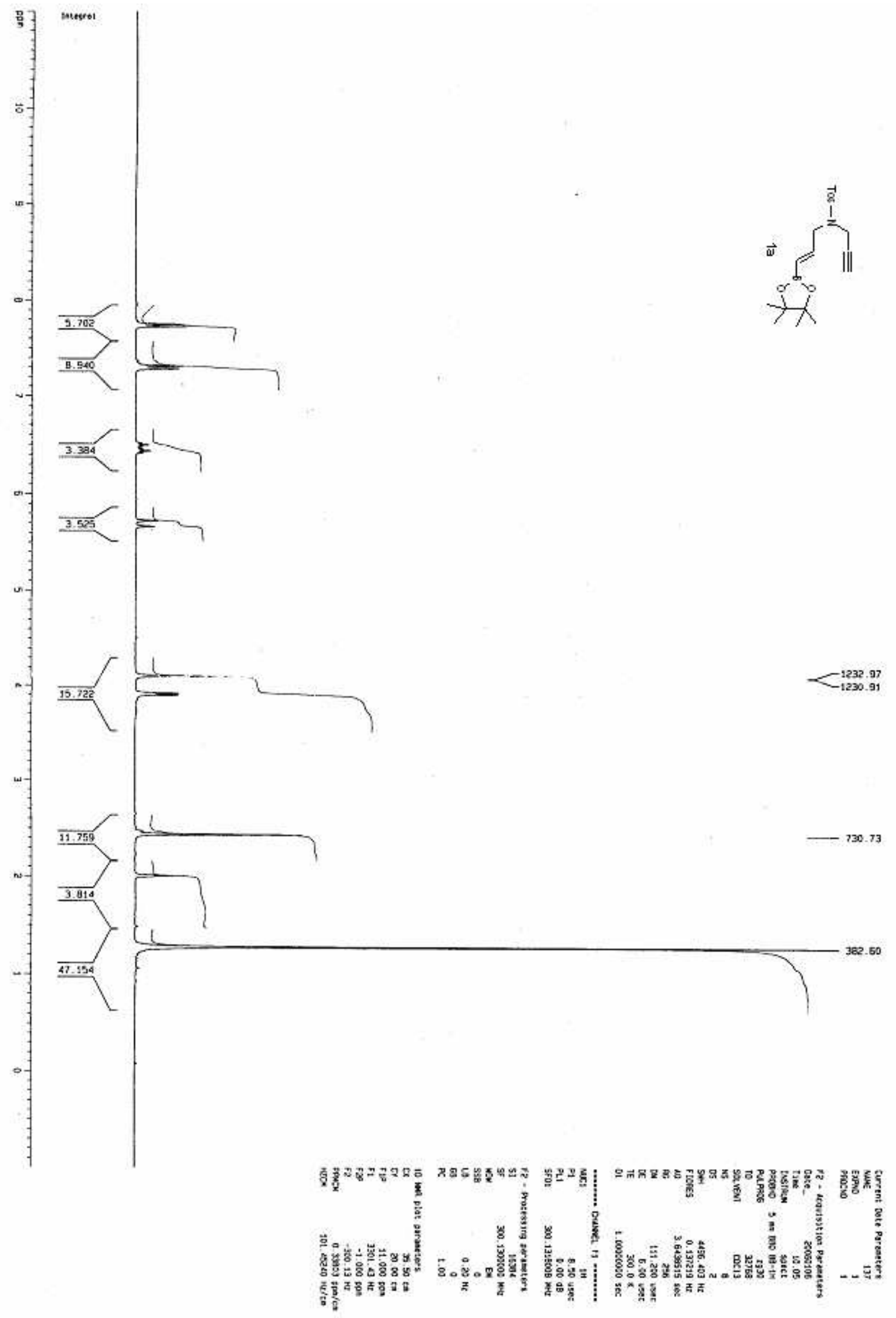




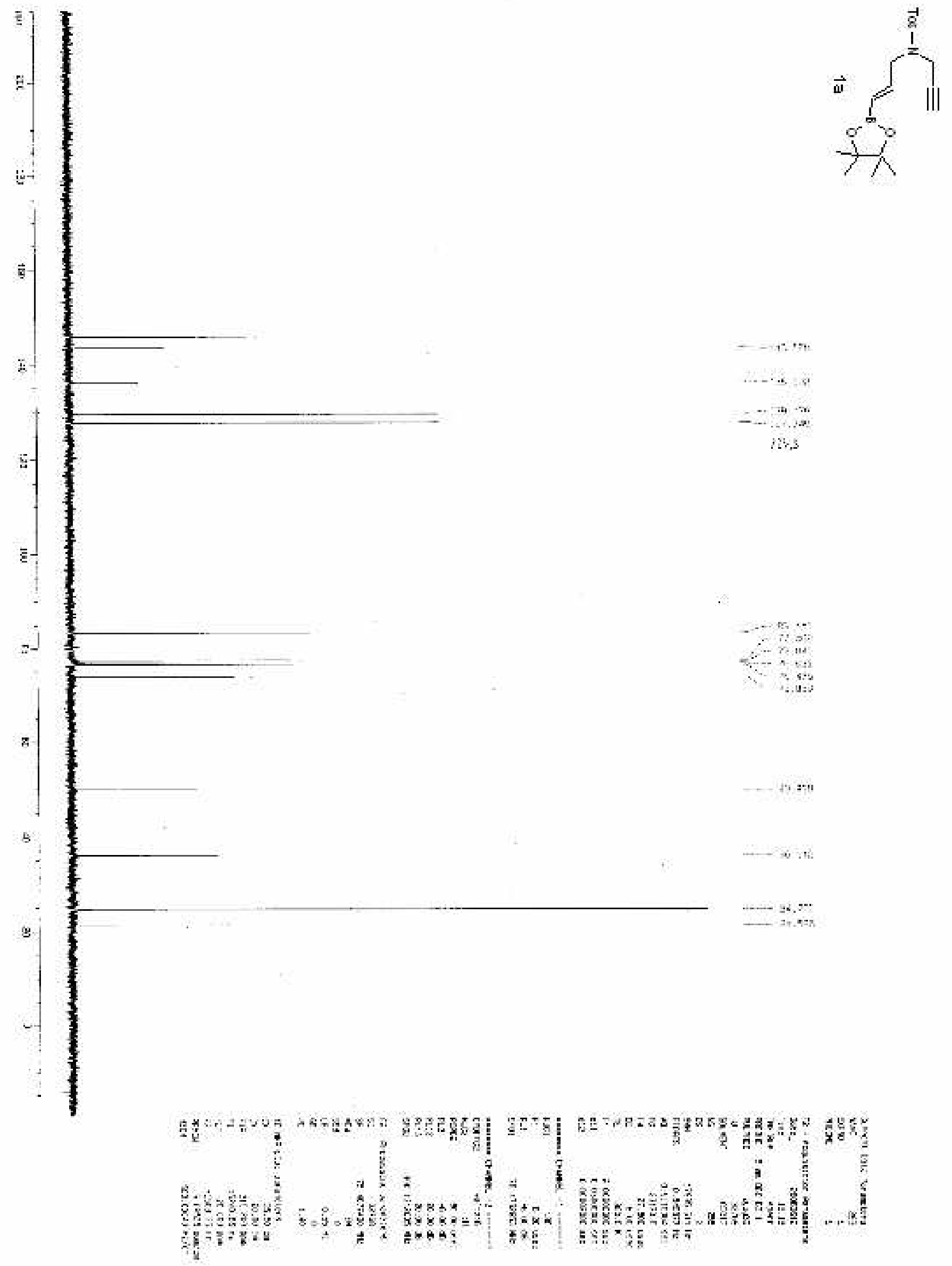

S-18 


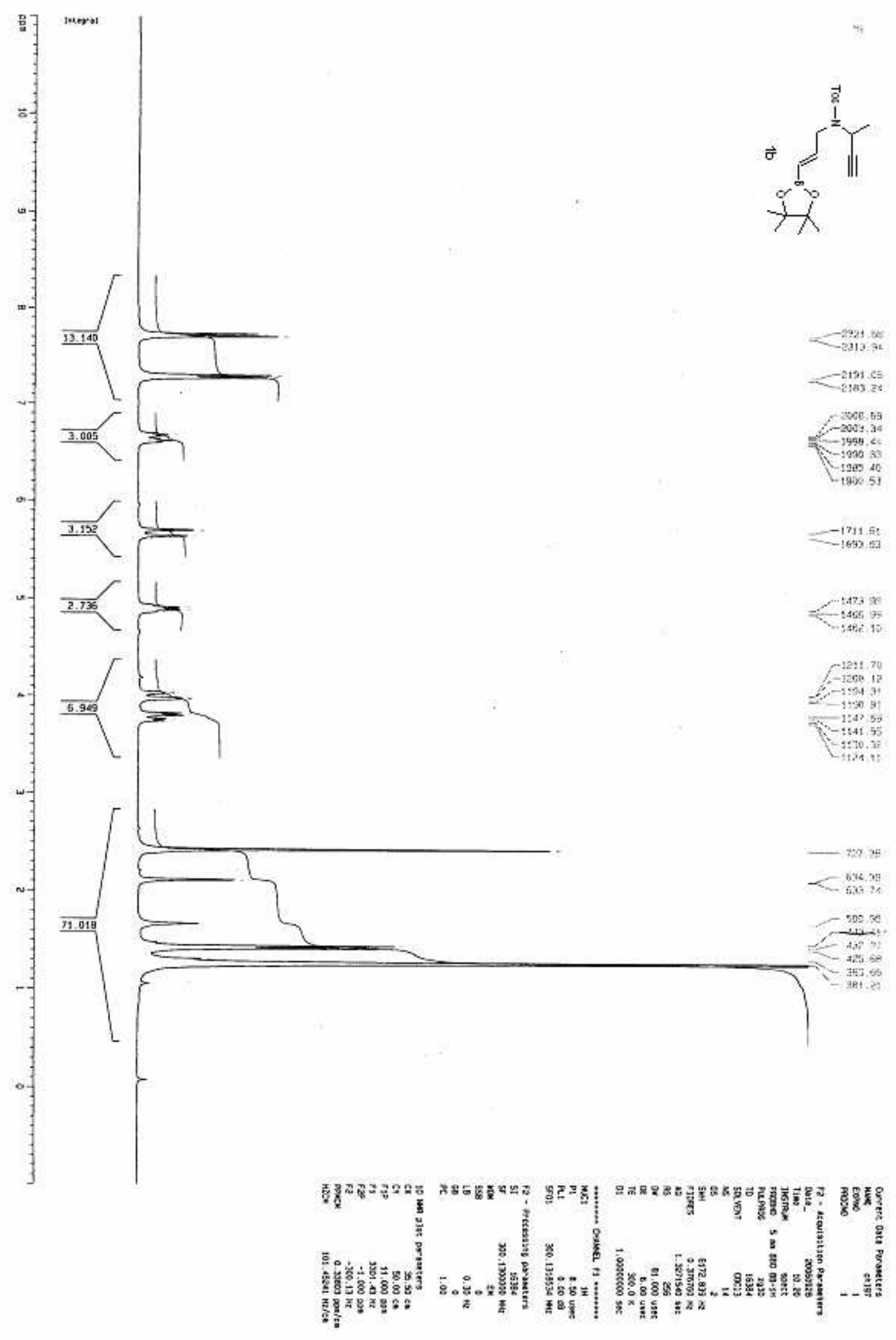




$$
1
$$



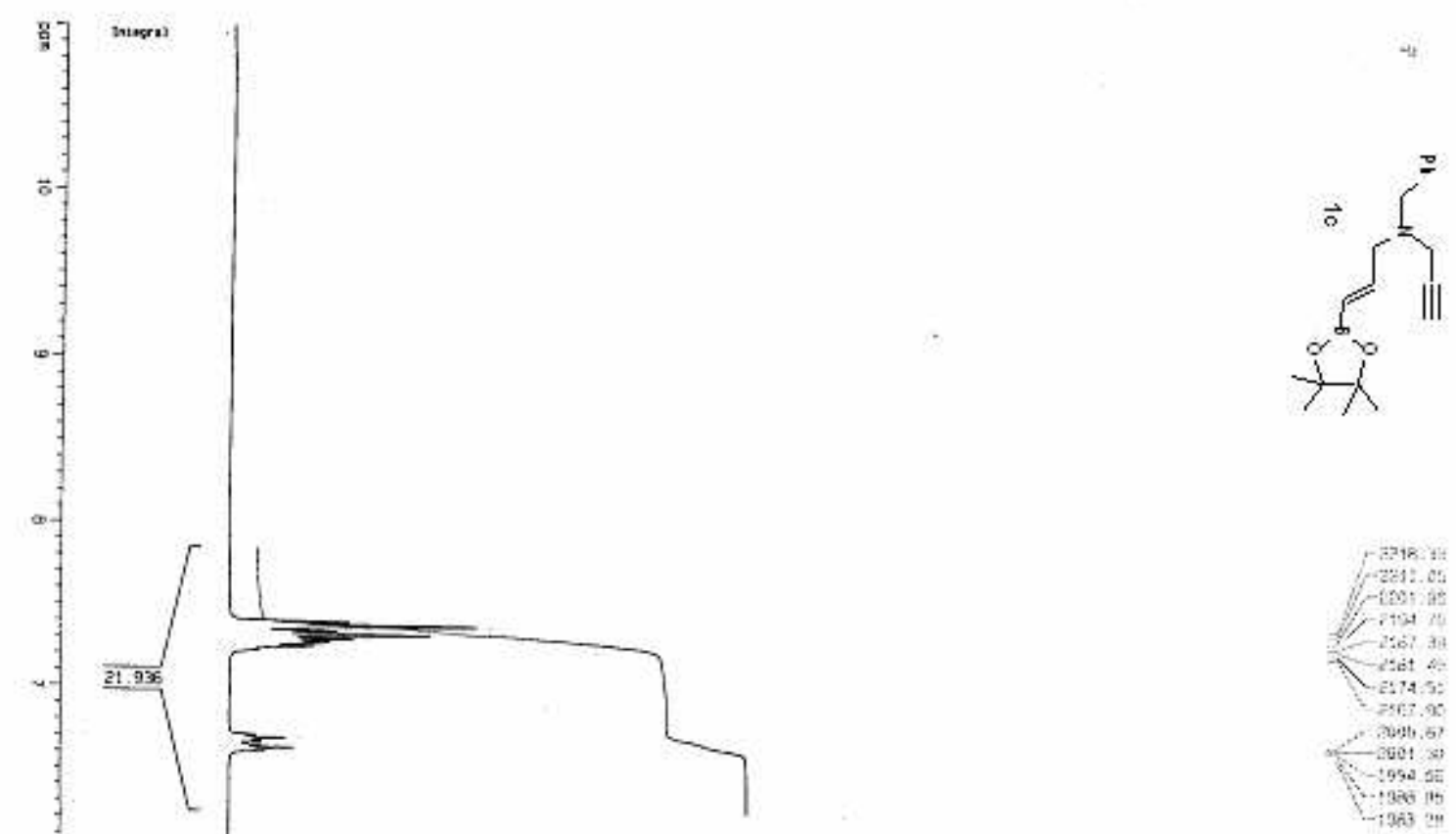

$-1 \times 3$
-129

- 2400 :

- -4243

3287
5135

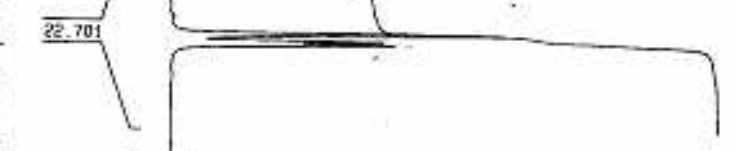

की गु

L En: 12

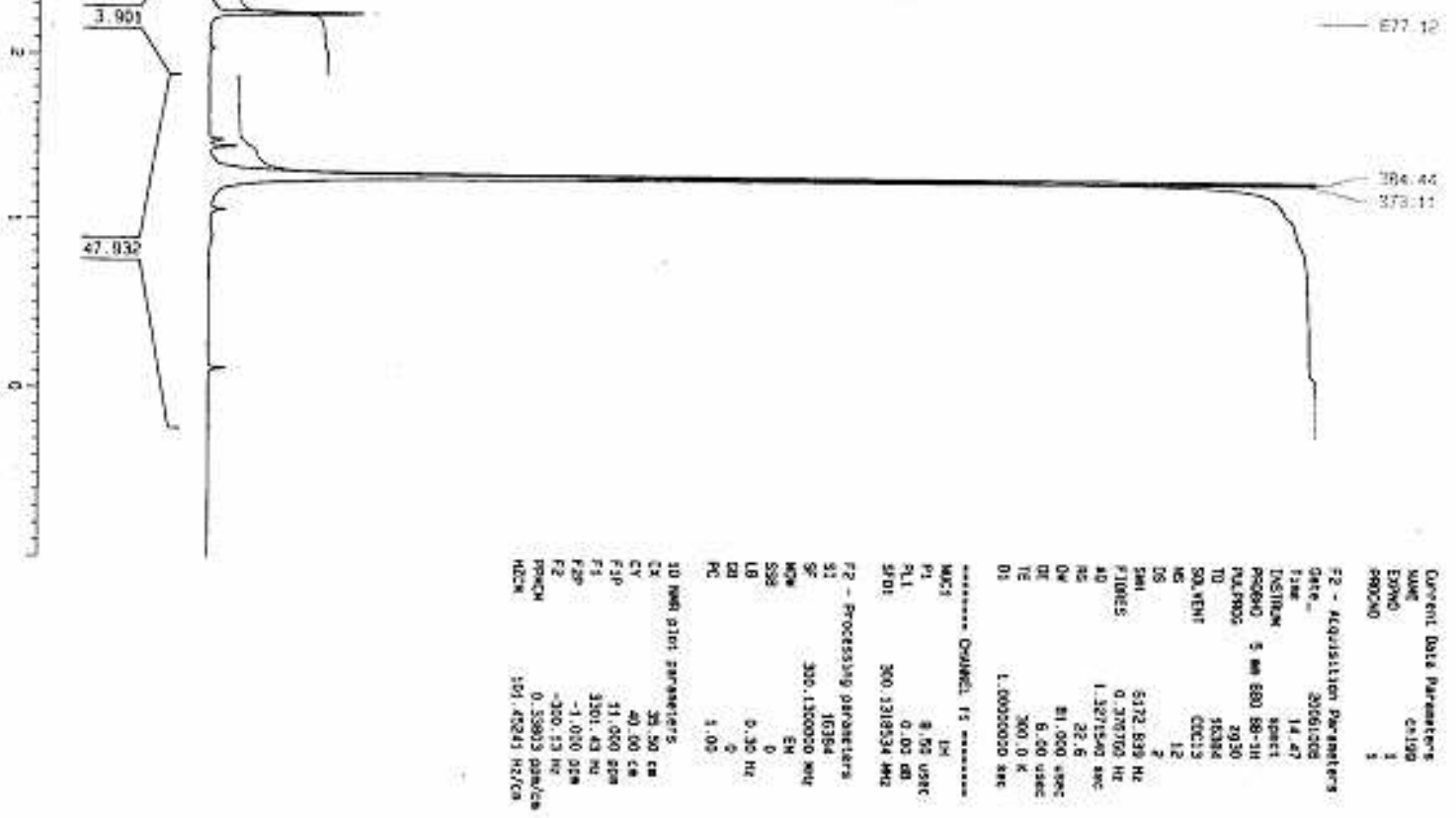




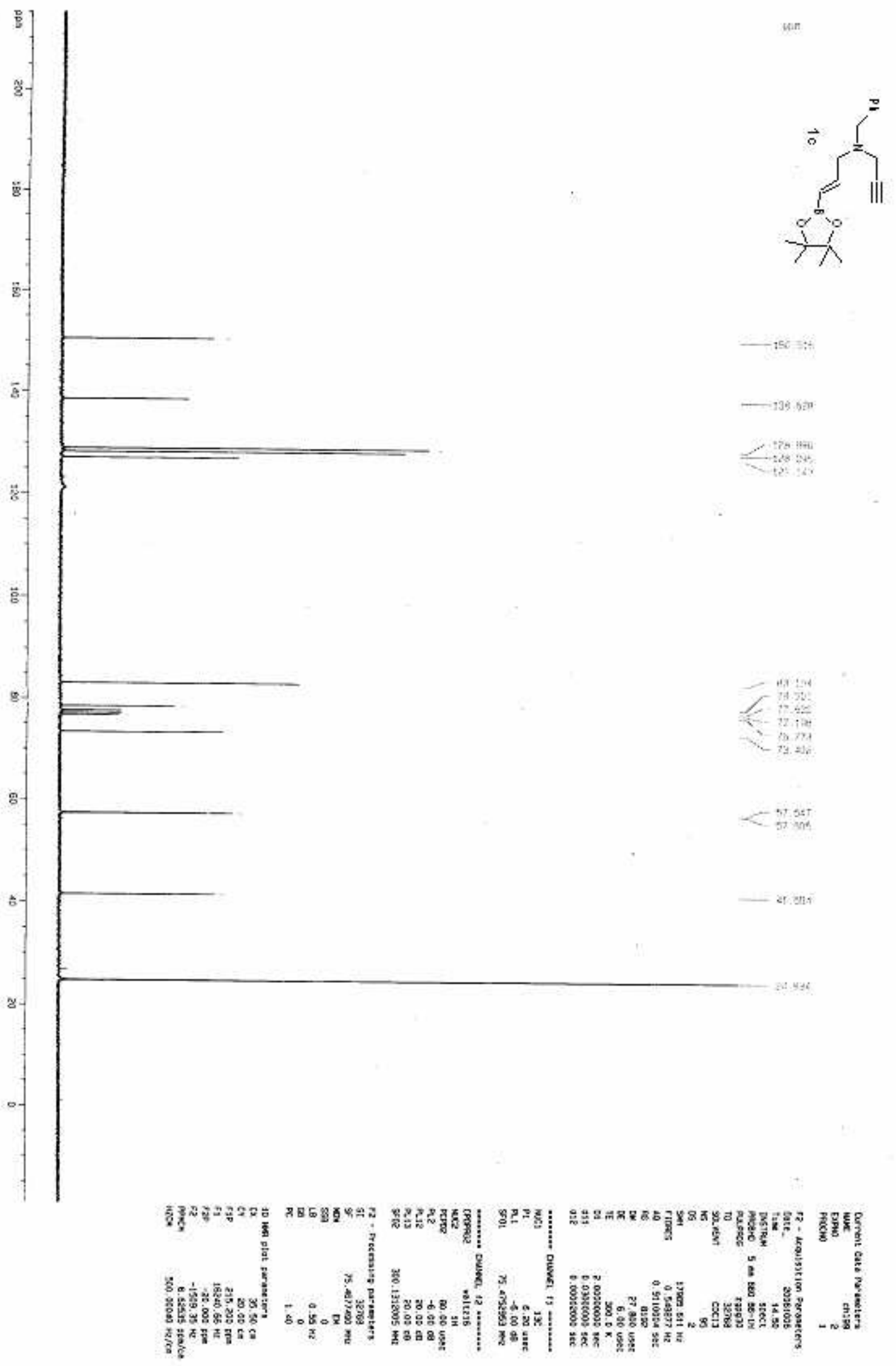




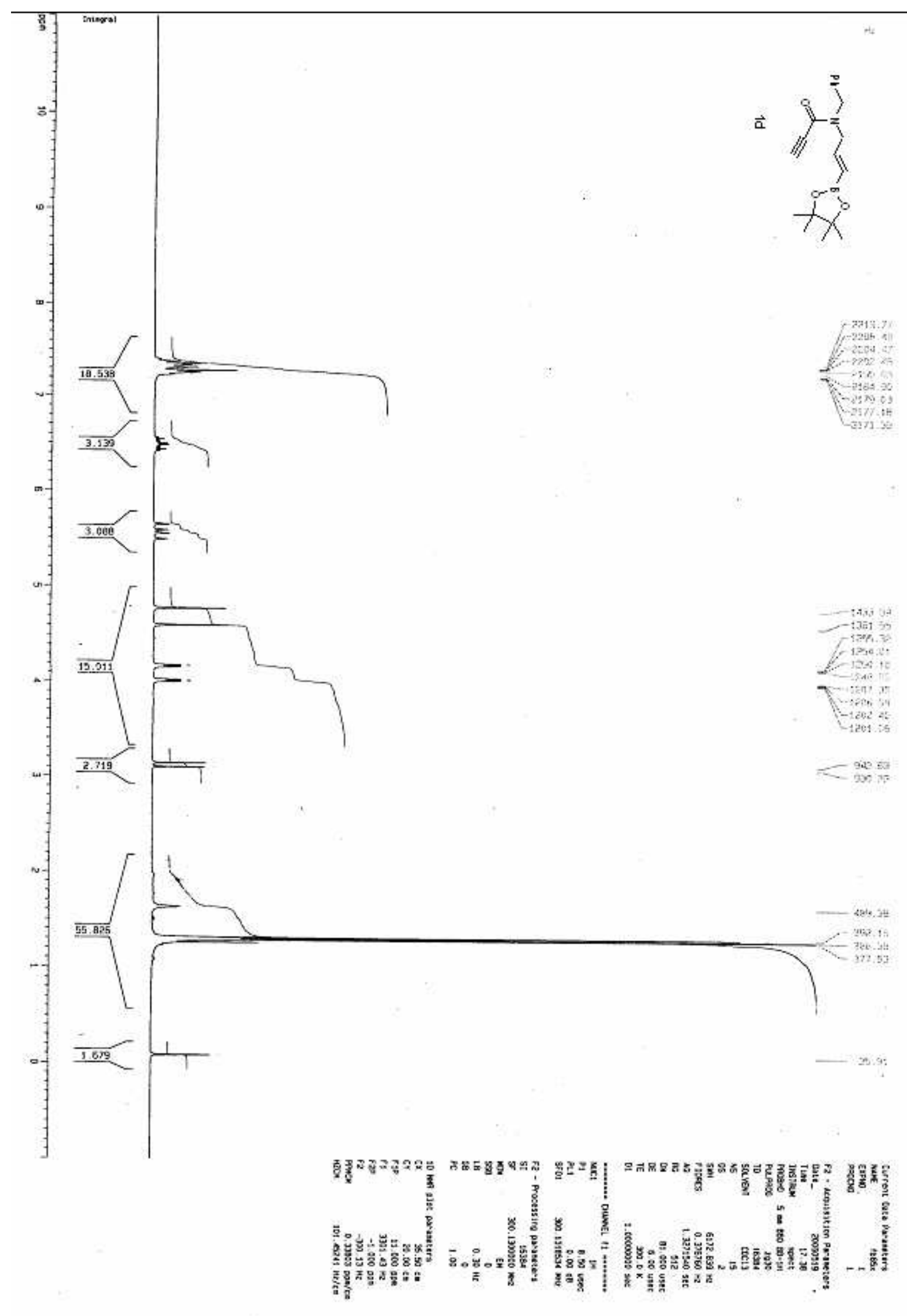




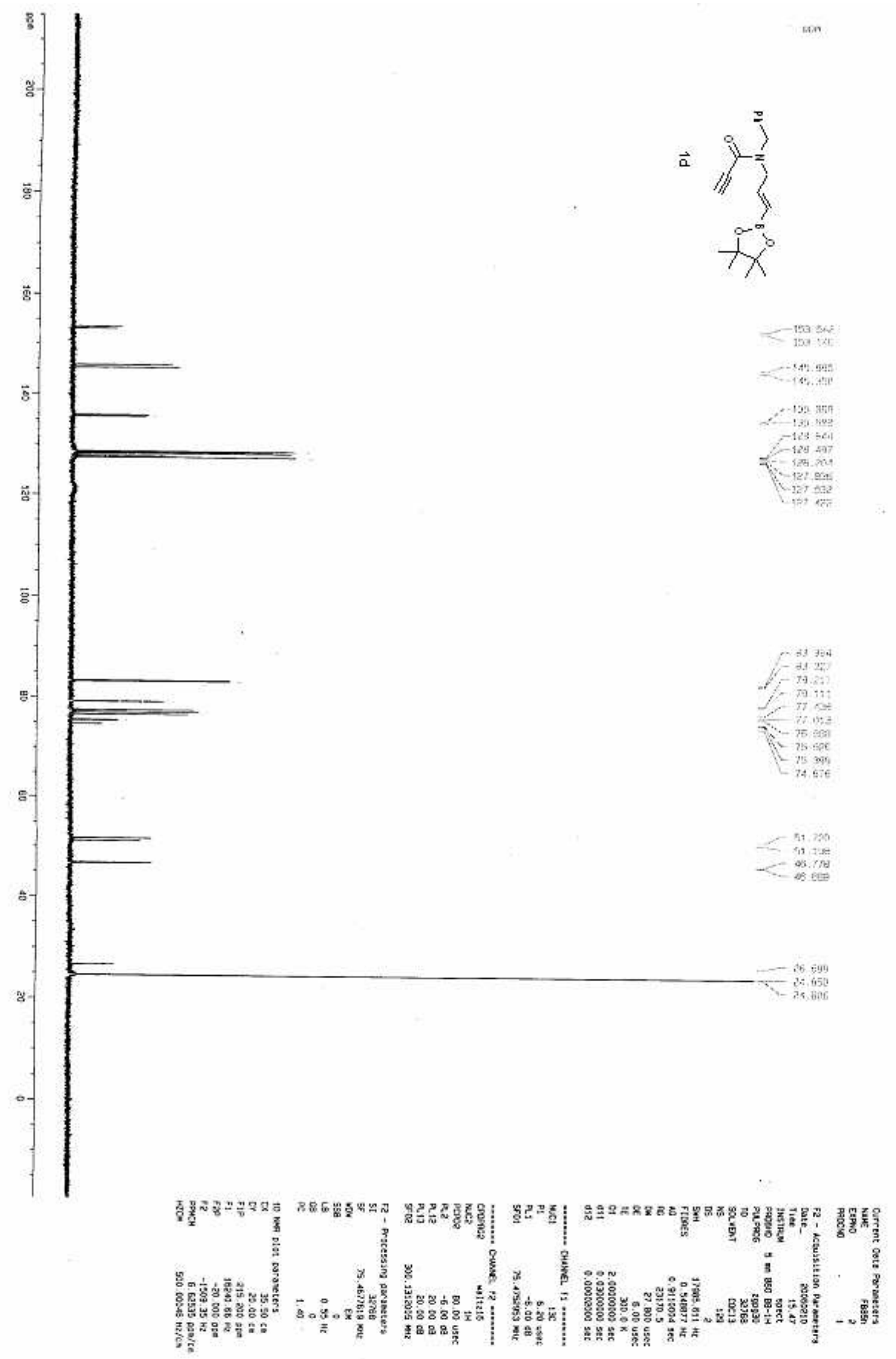




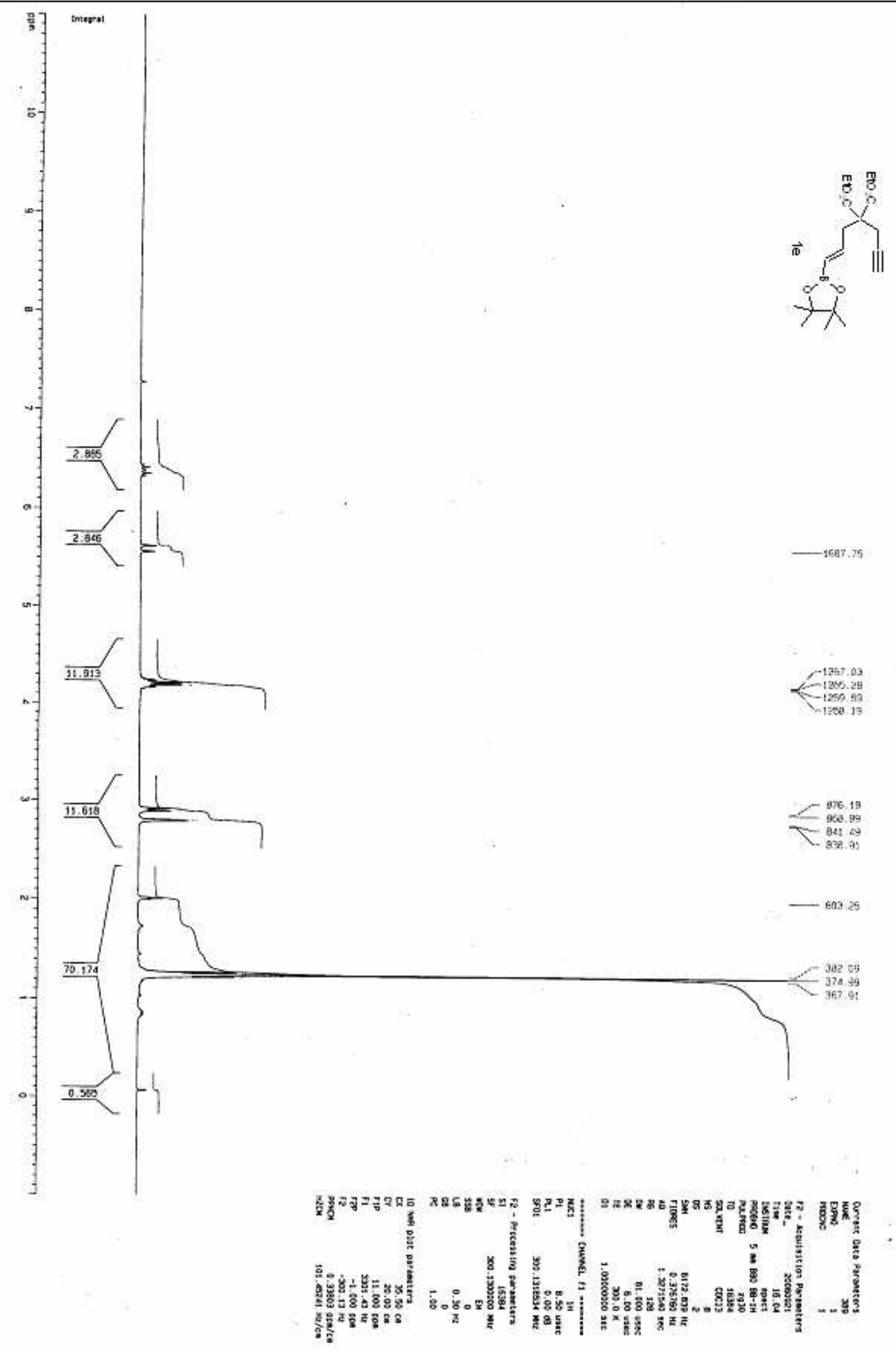



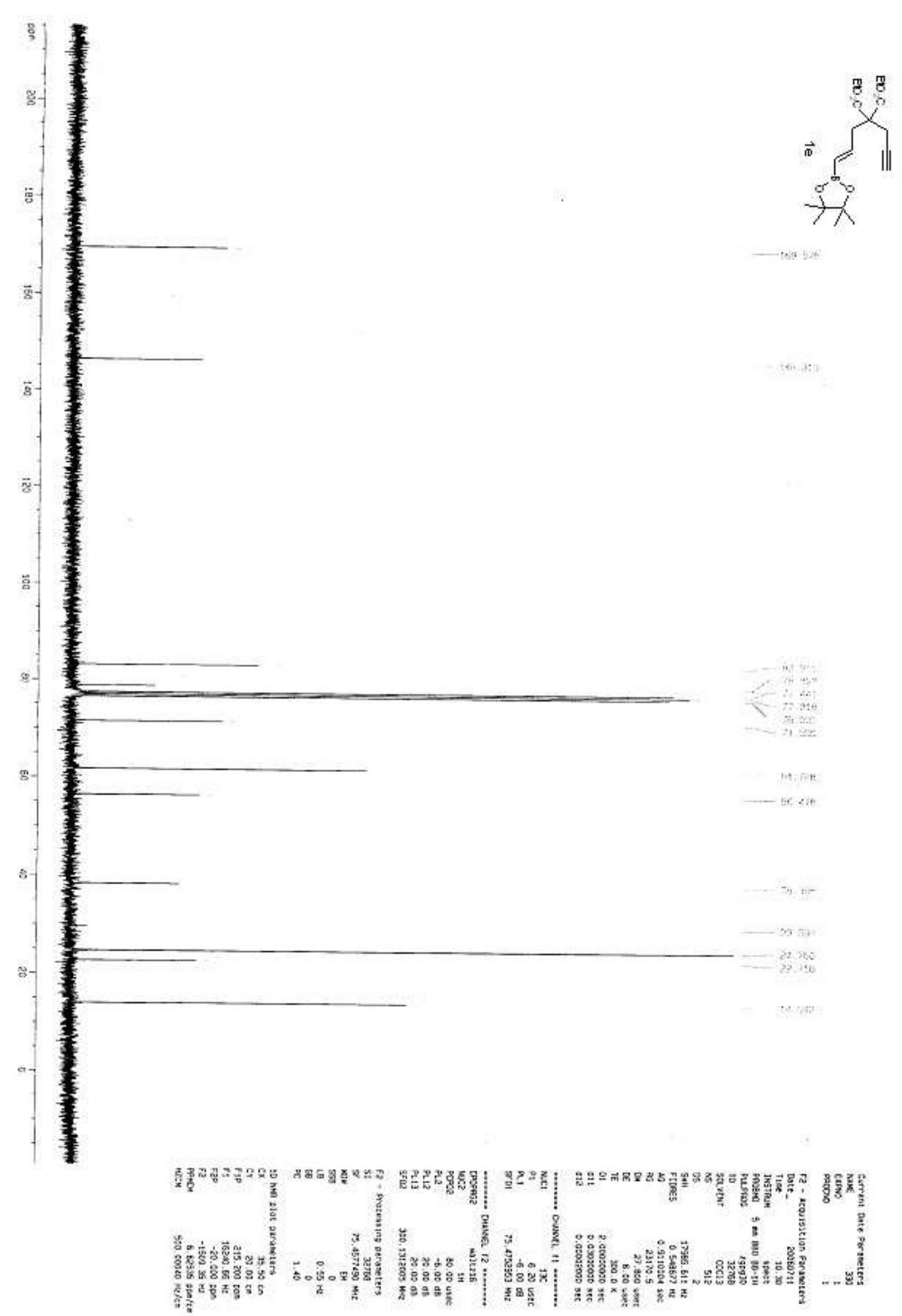


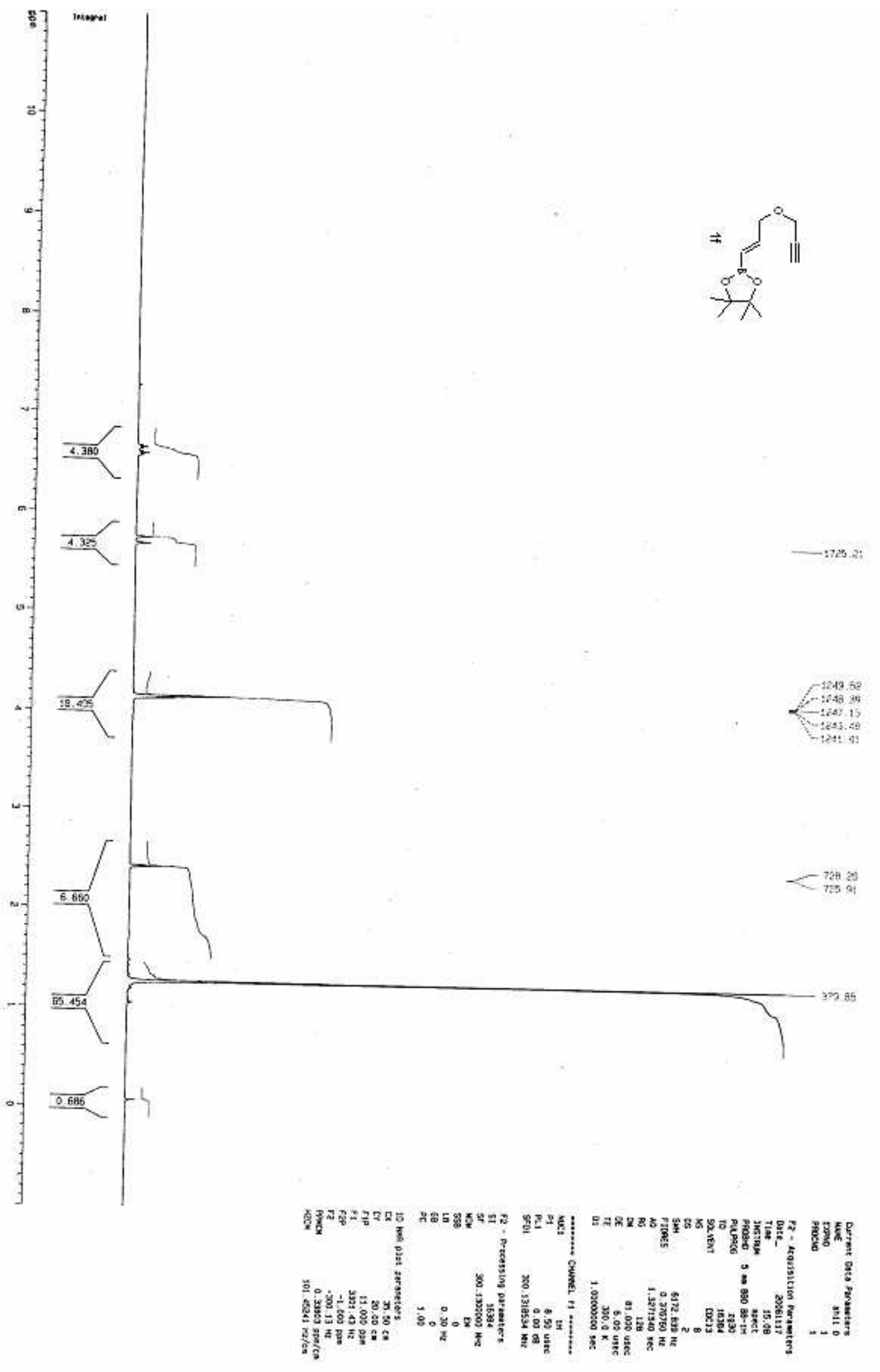




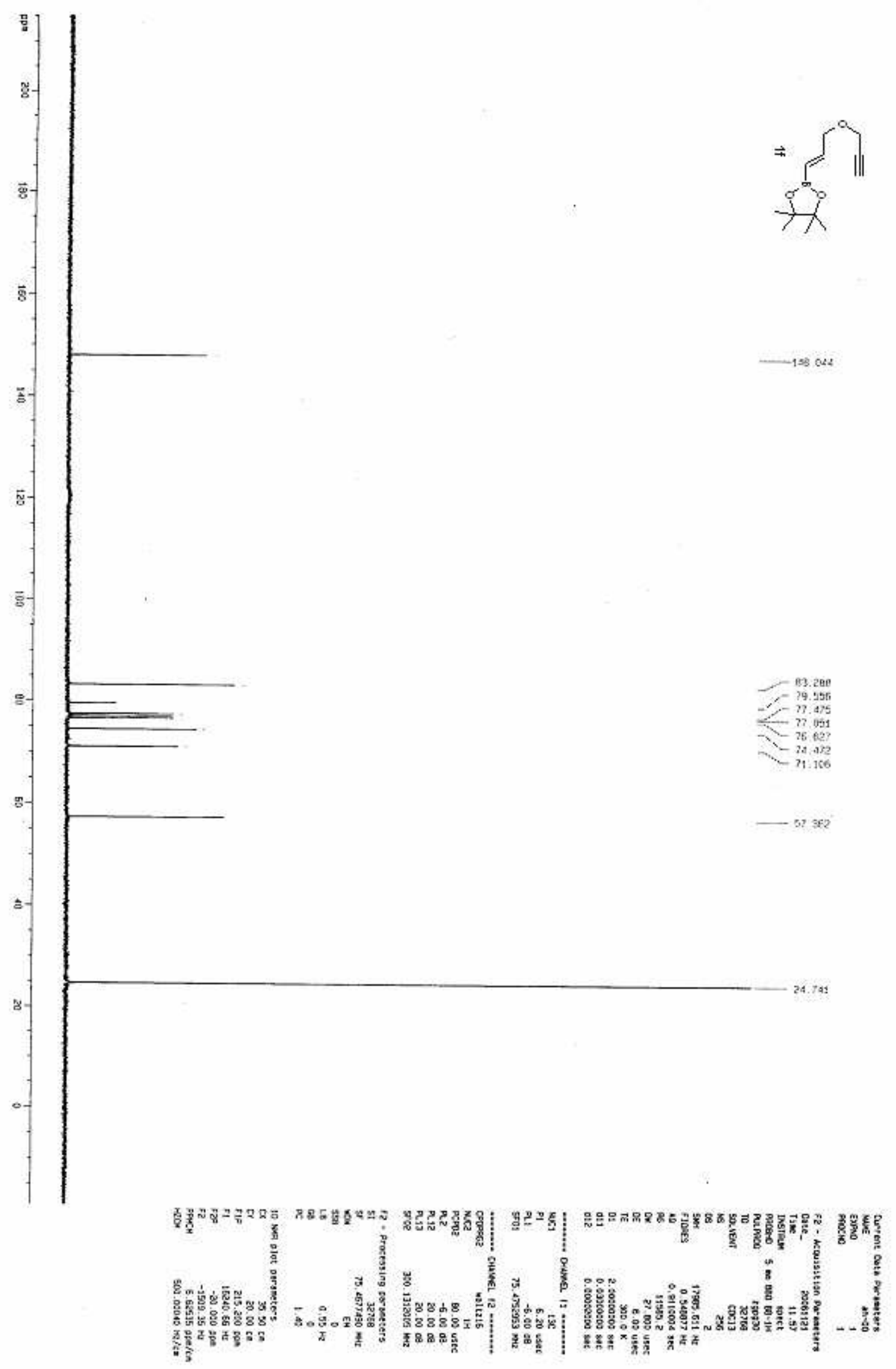




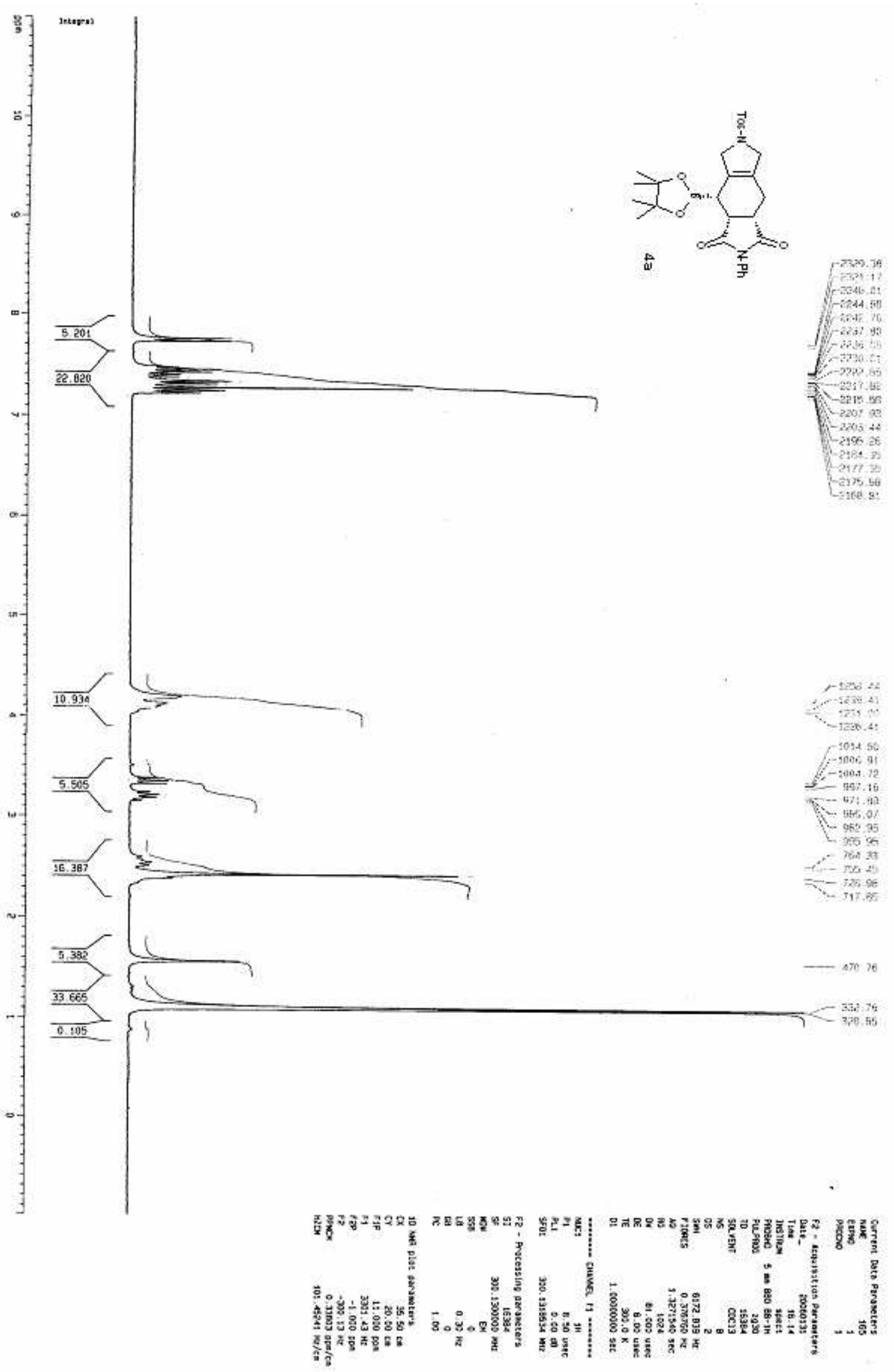



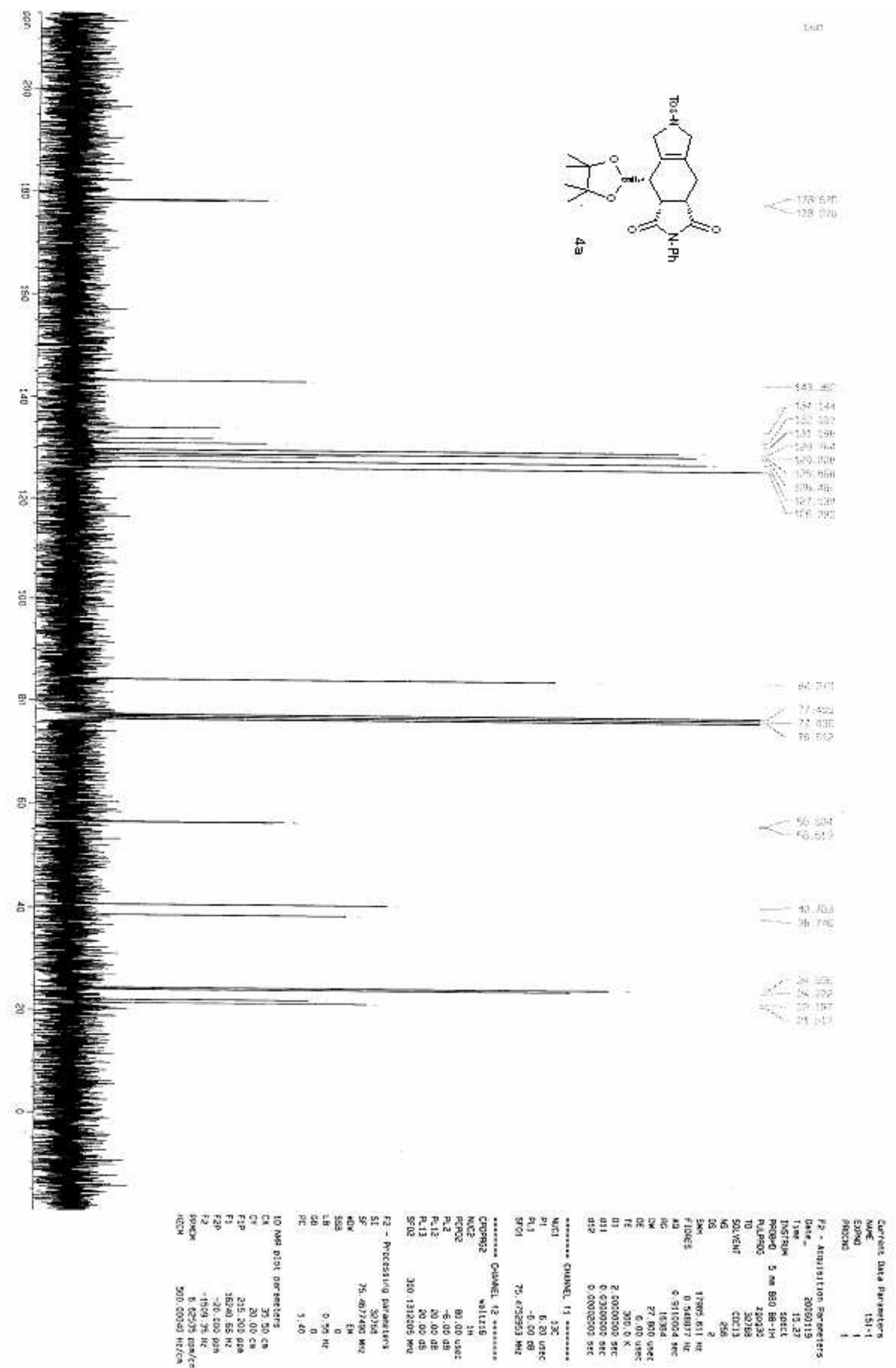


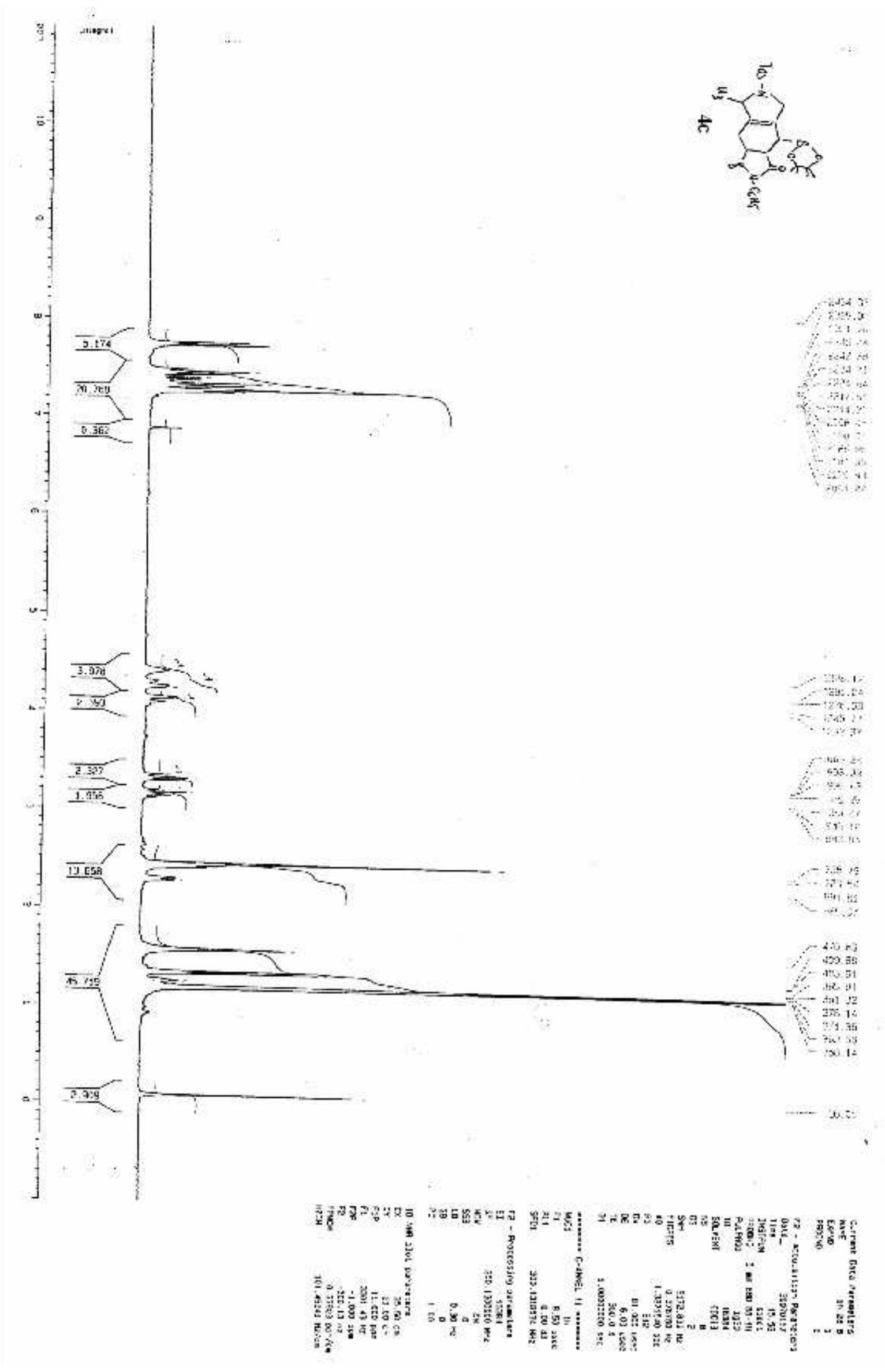




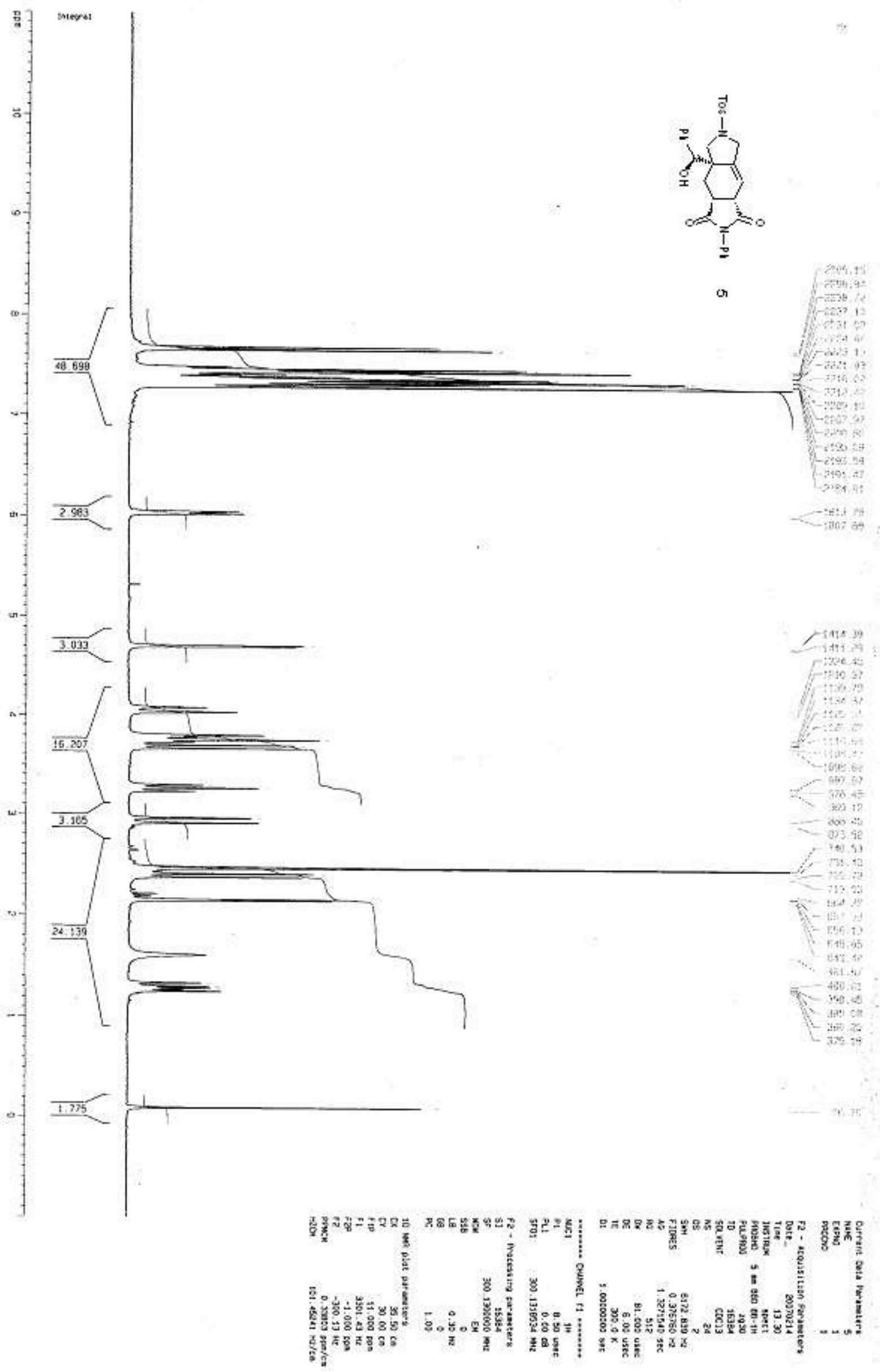




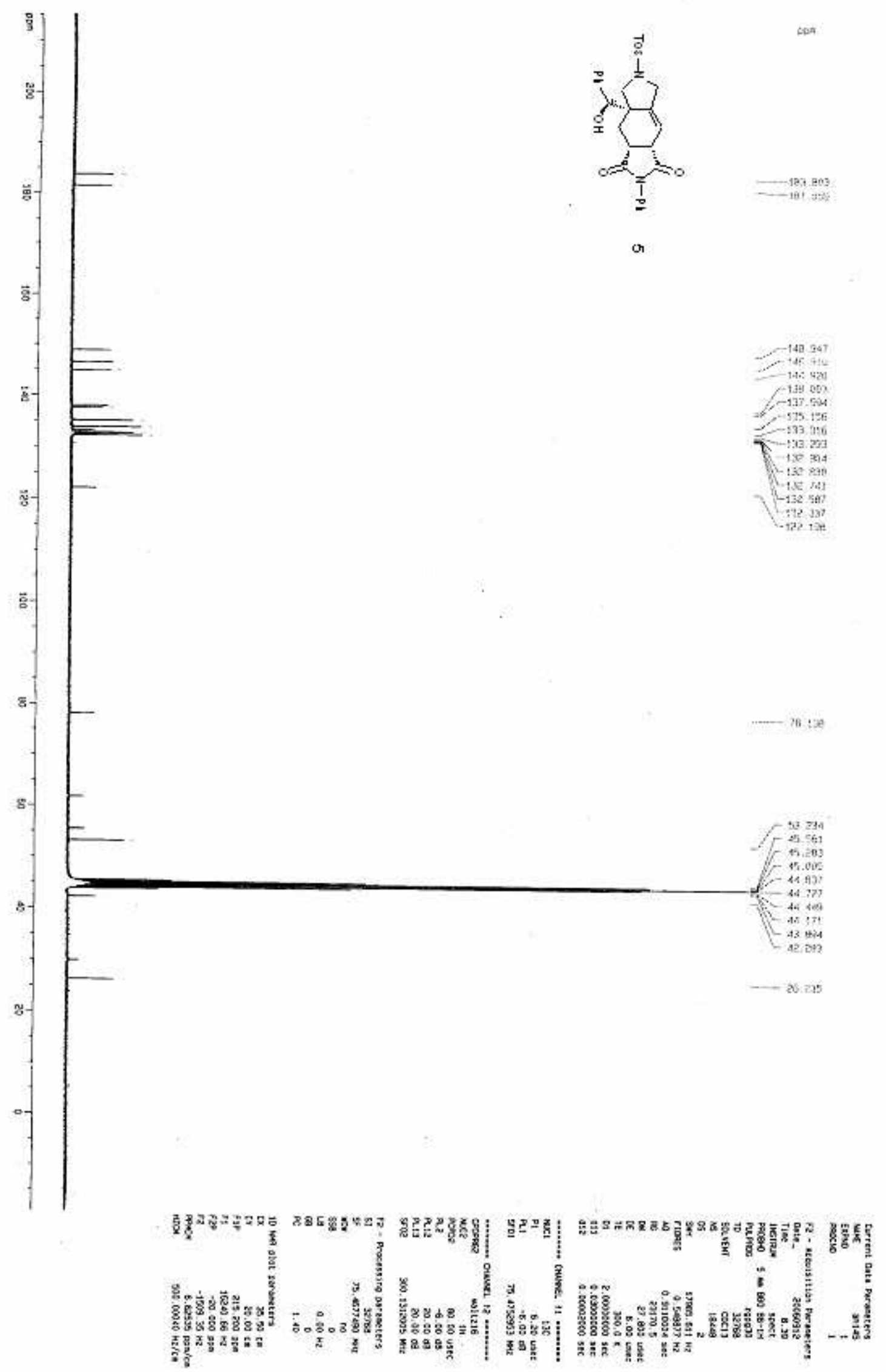




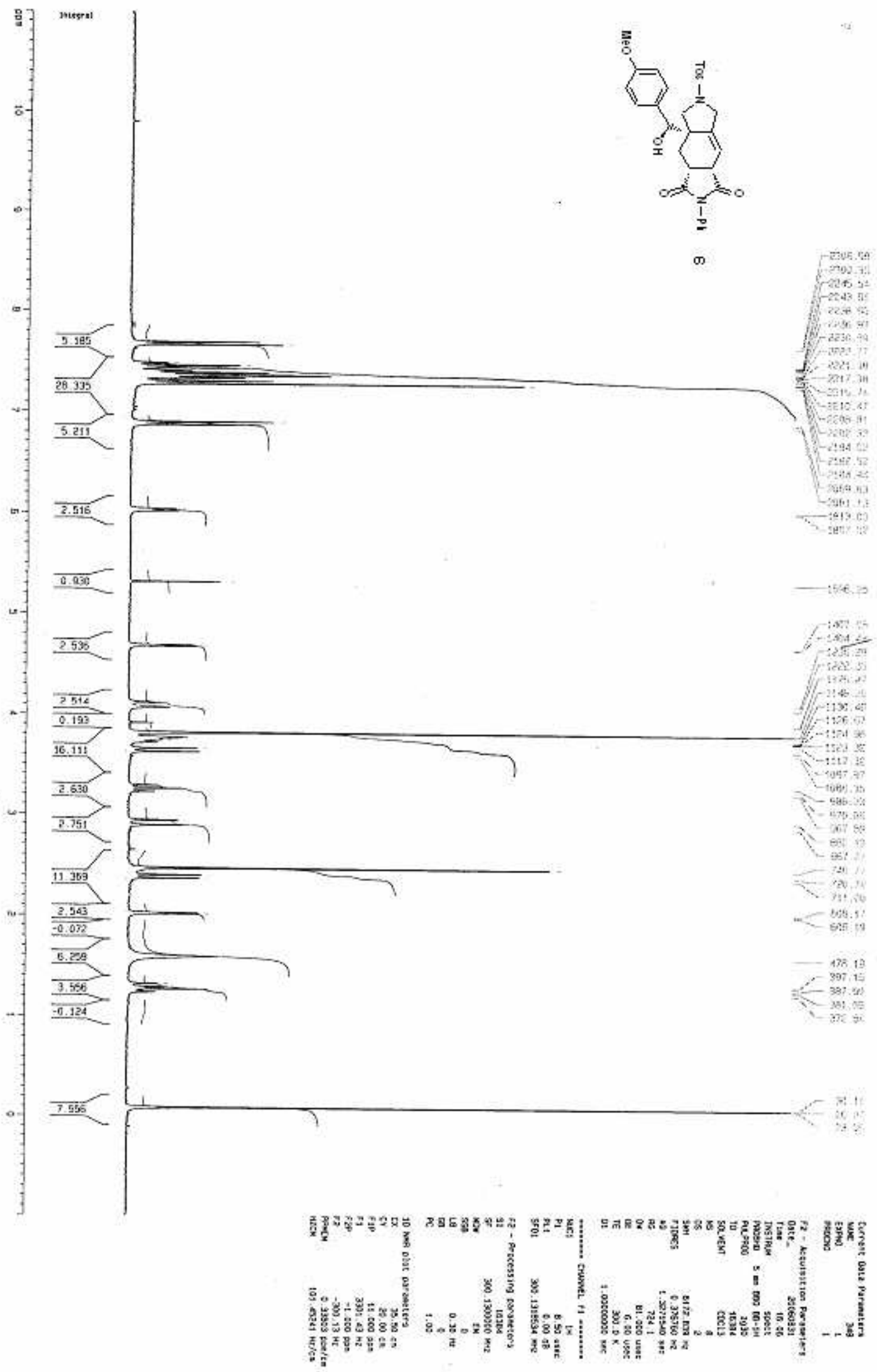




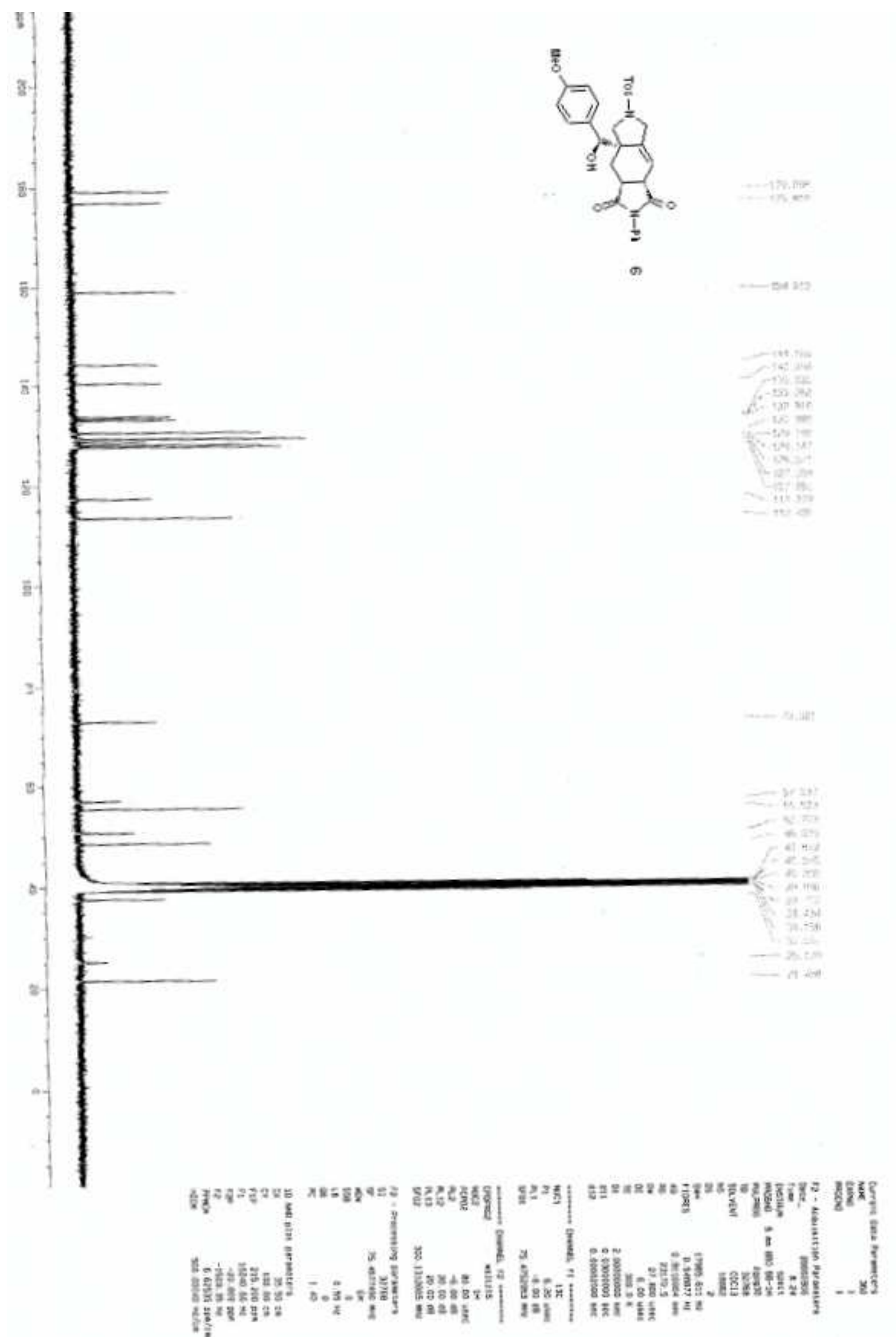

S-35 


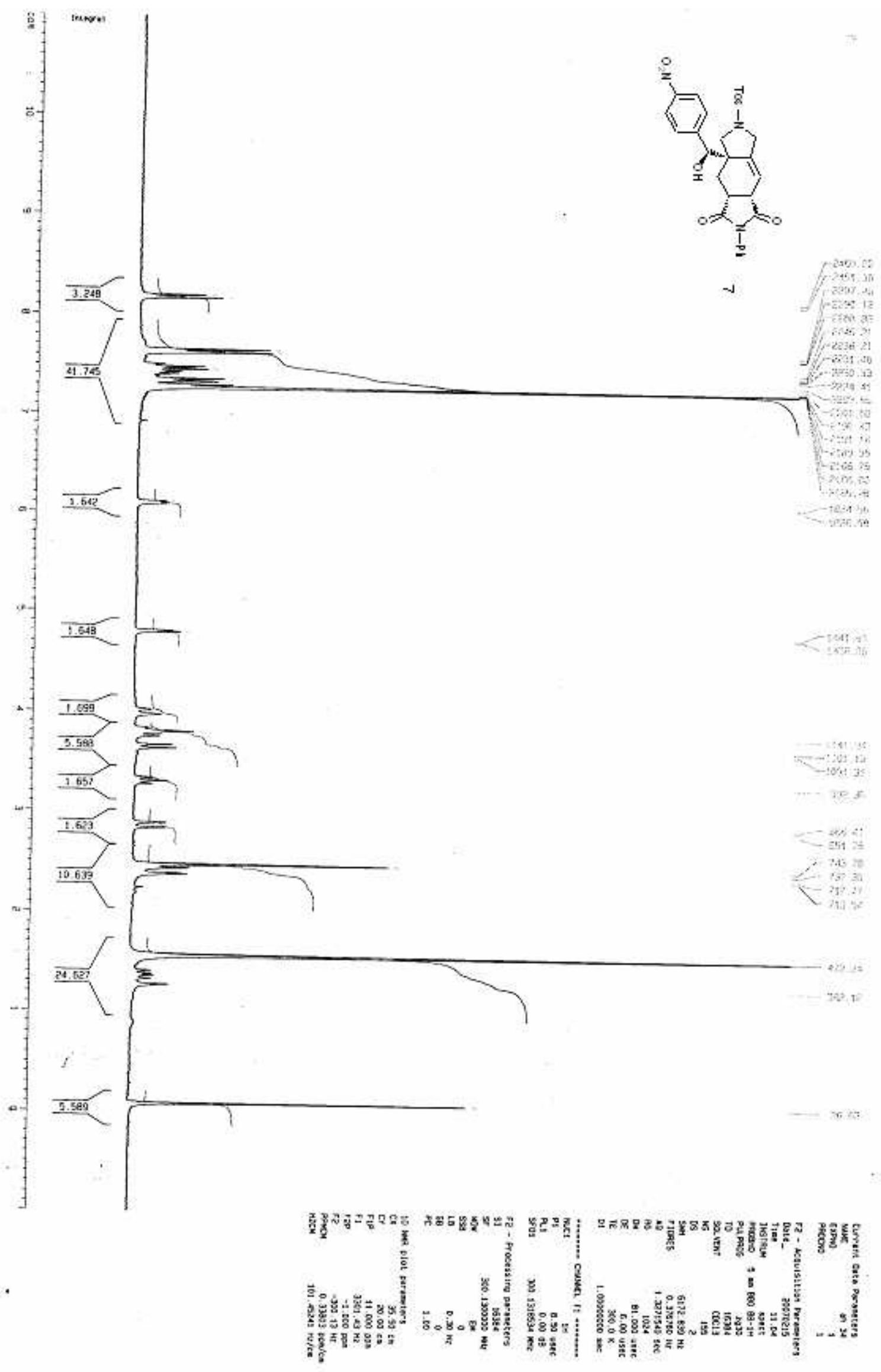




$$
E
$$



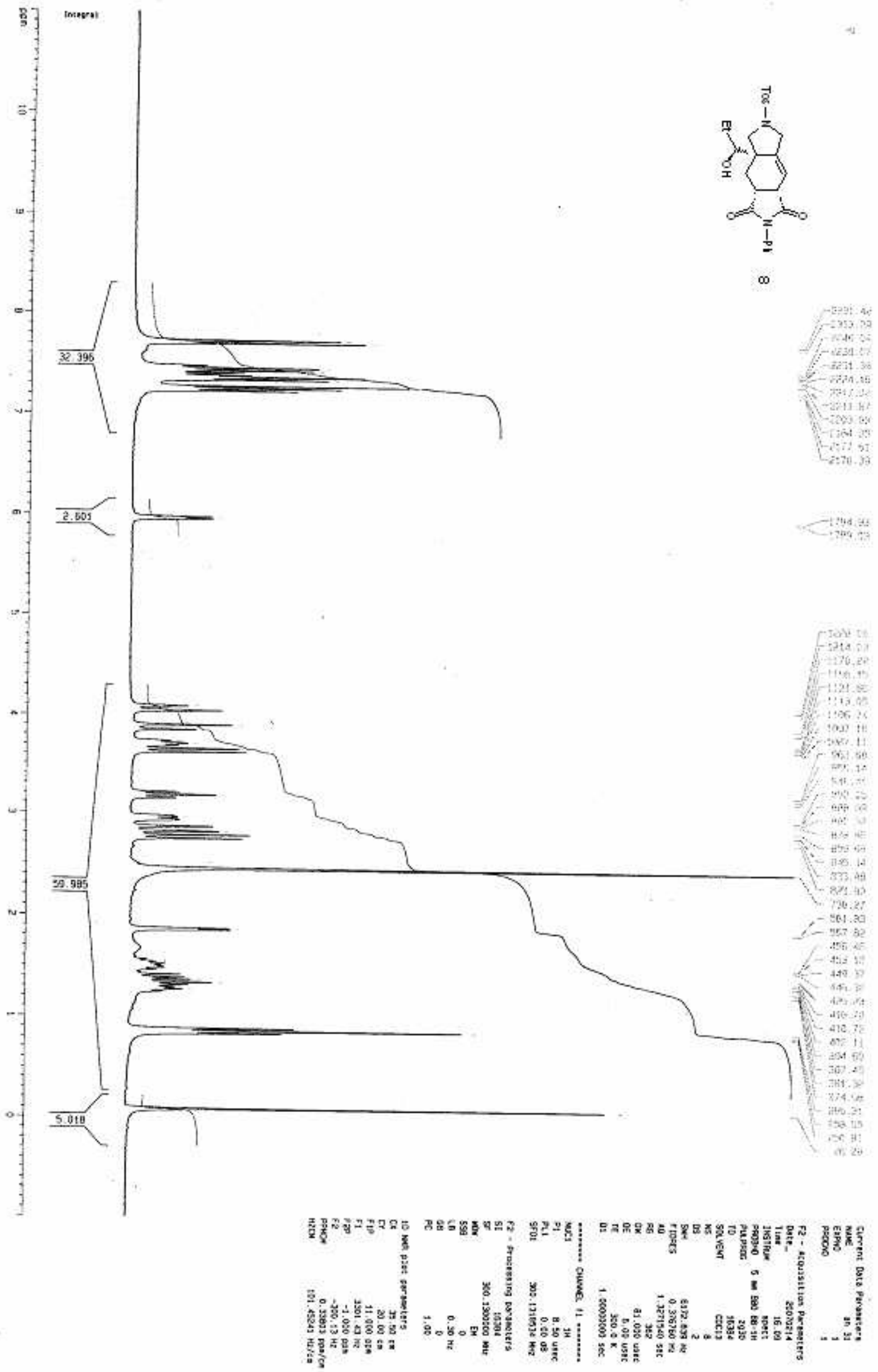


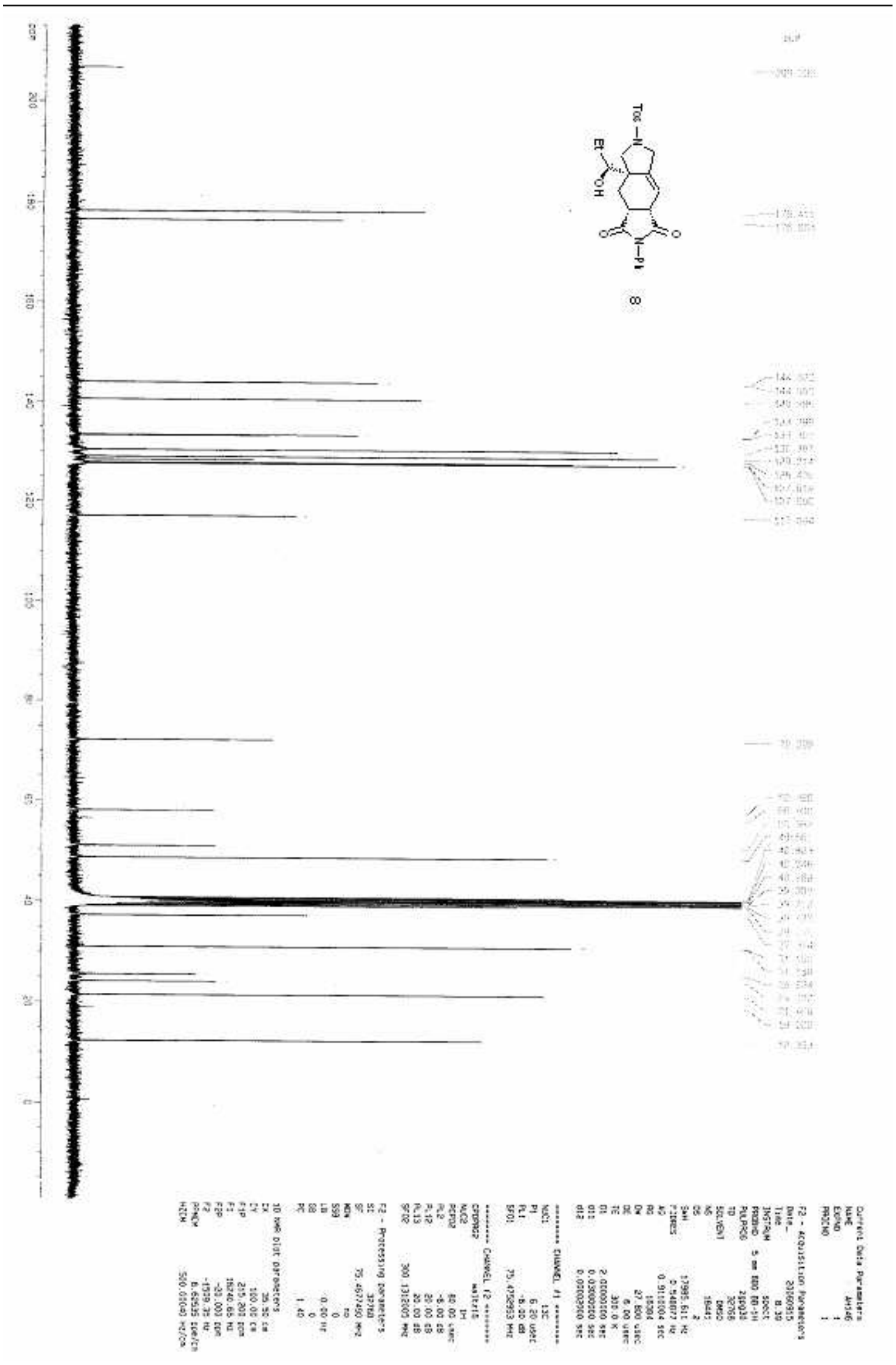




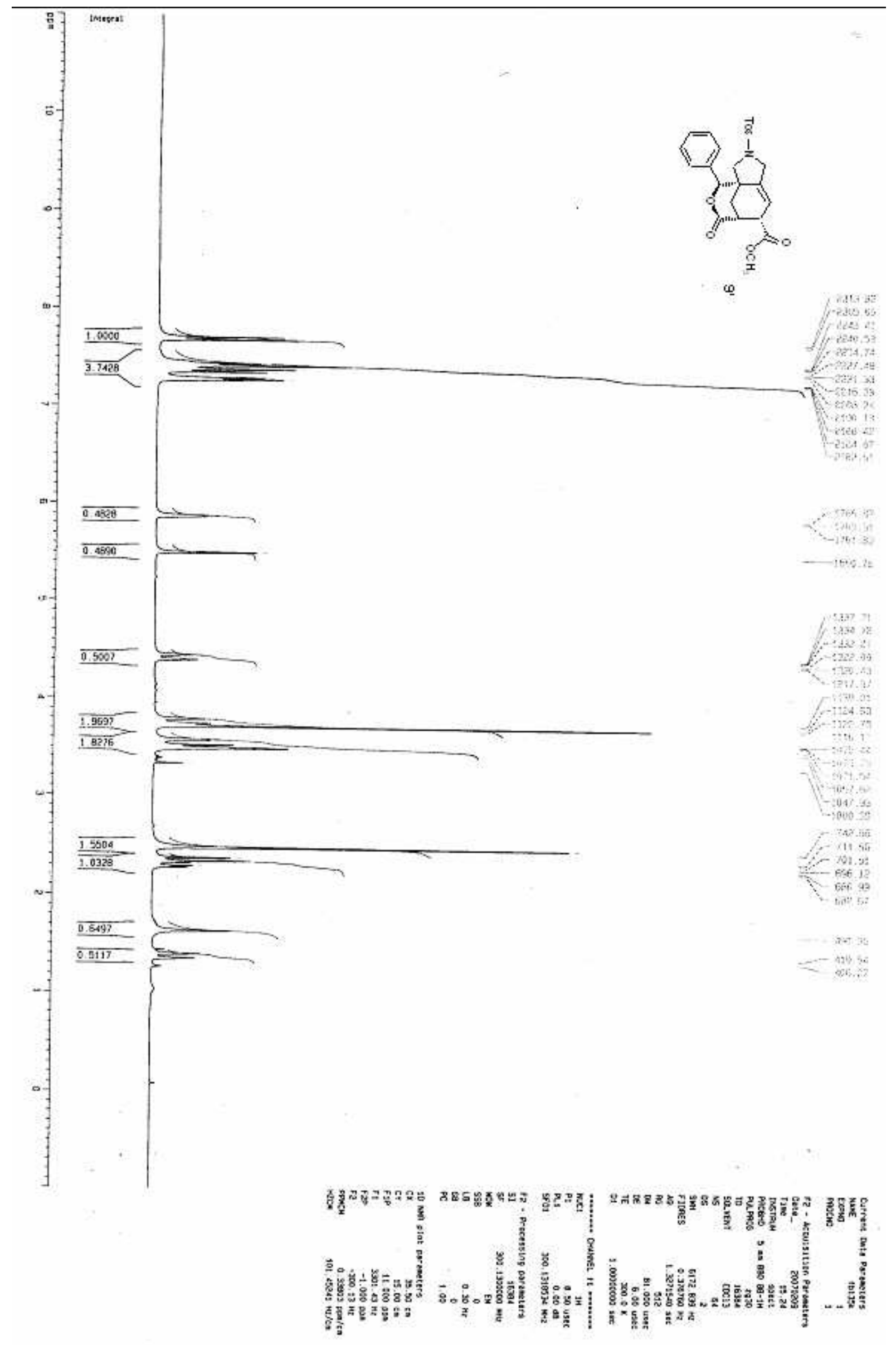




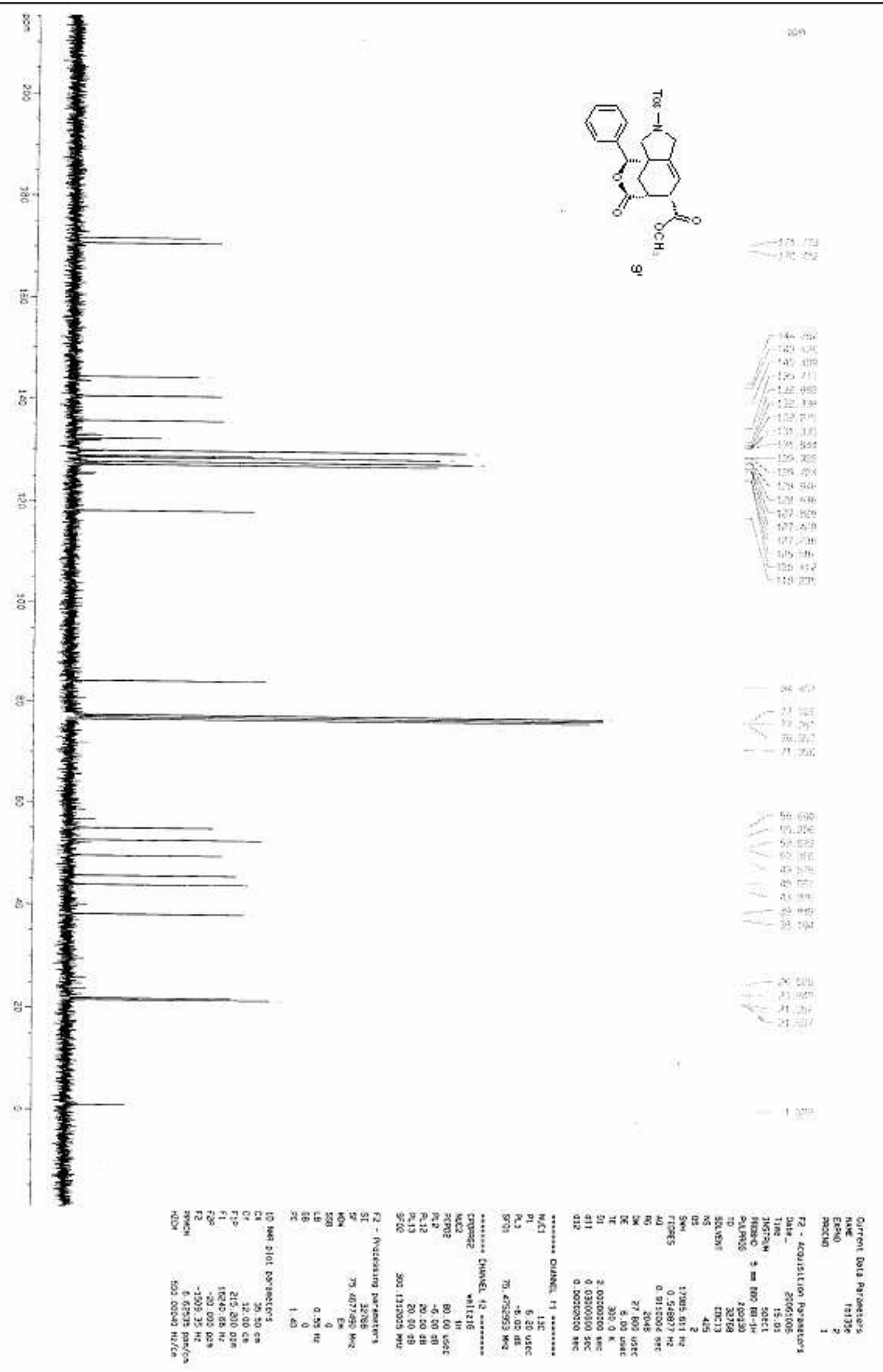




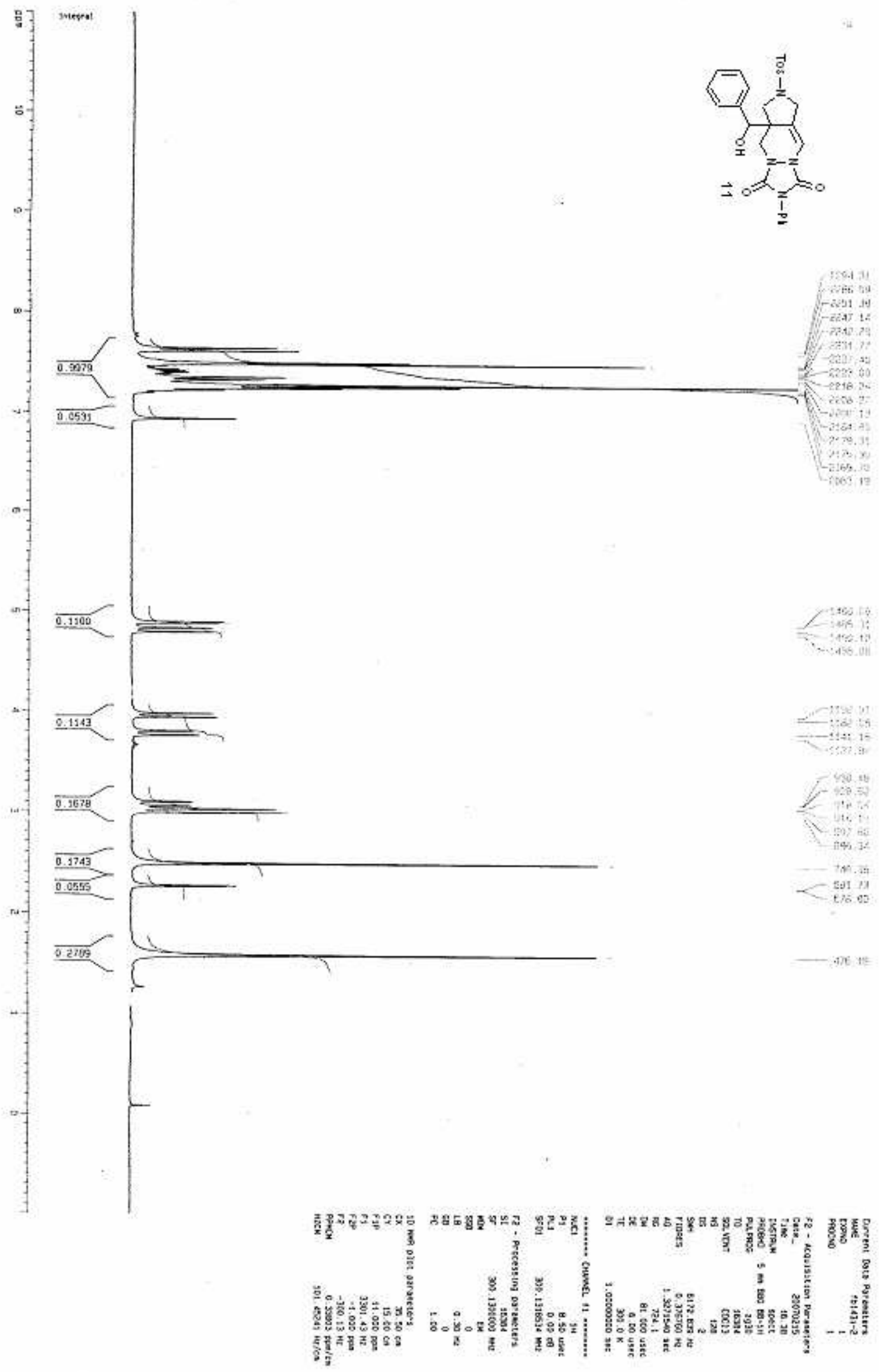




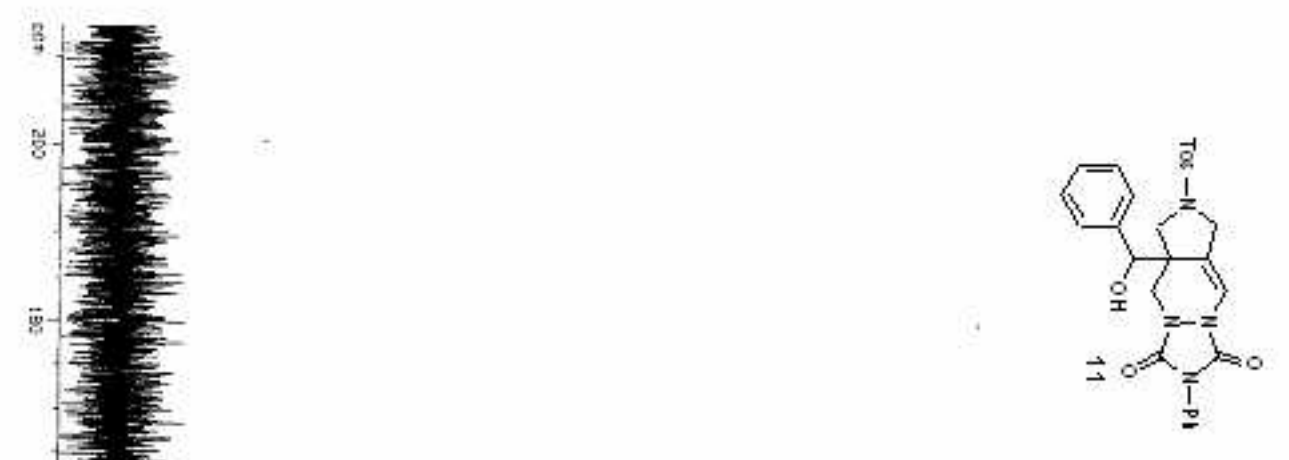

咅

s-

명

b-

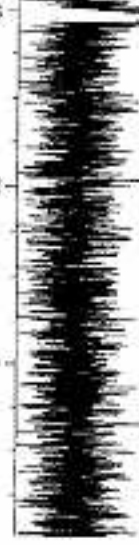

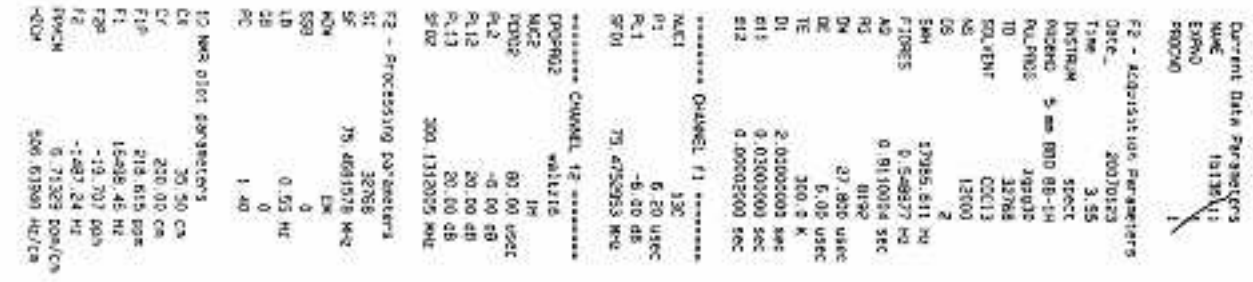



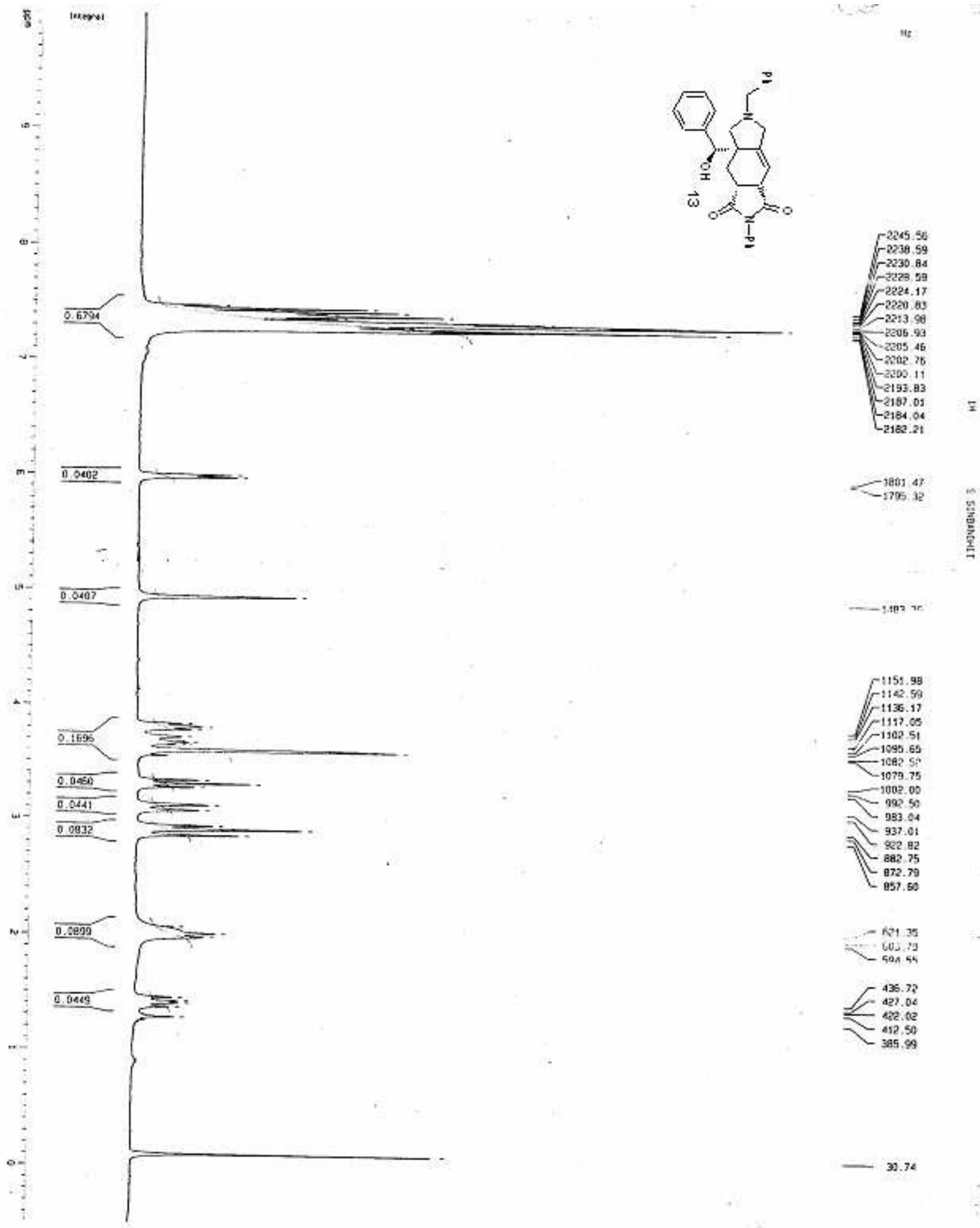

-

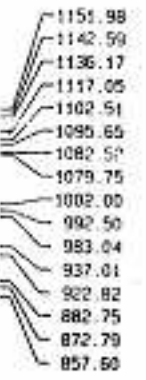

. - N 35

602.79
-50055

435.39

ace 0

-42.50
-395.99

- 35 क

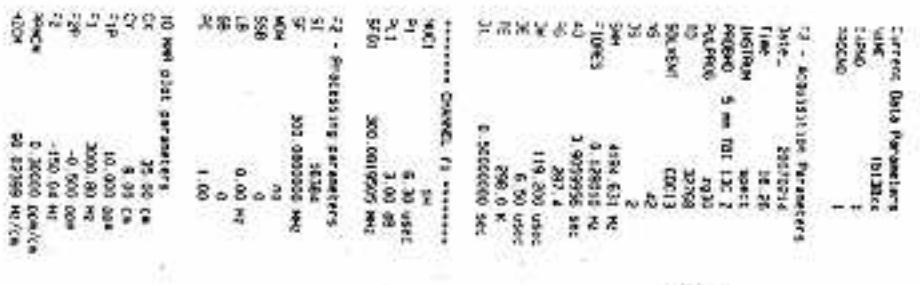



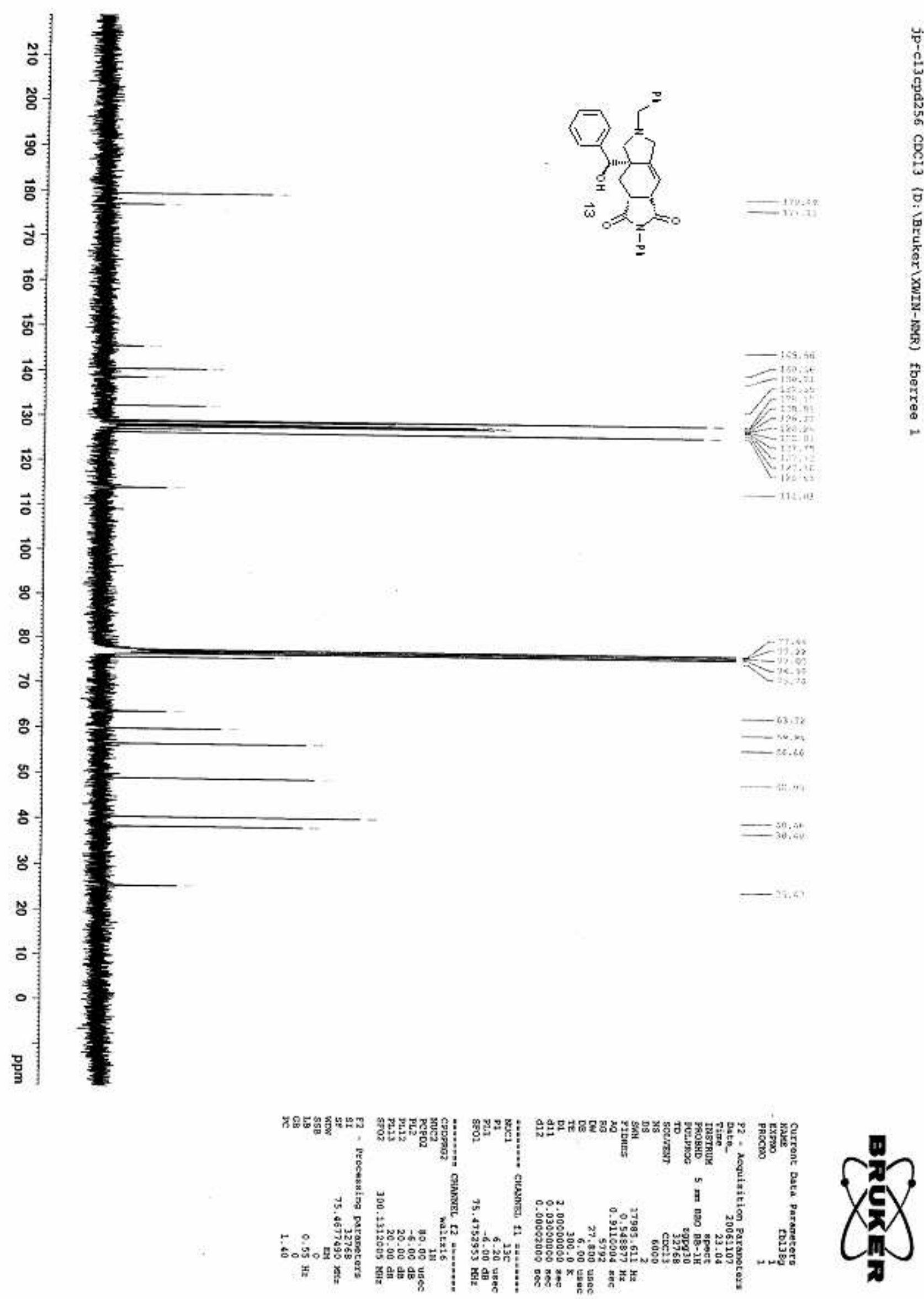

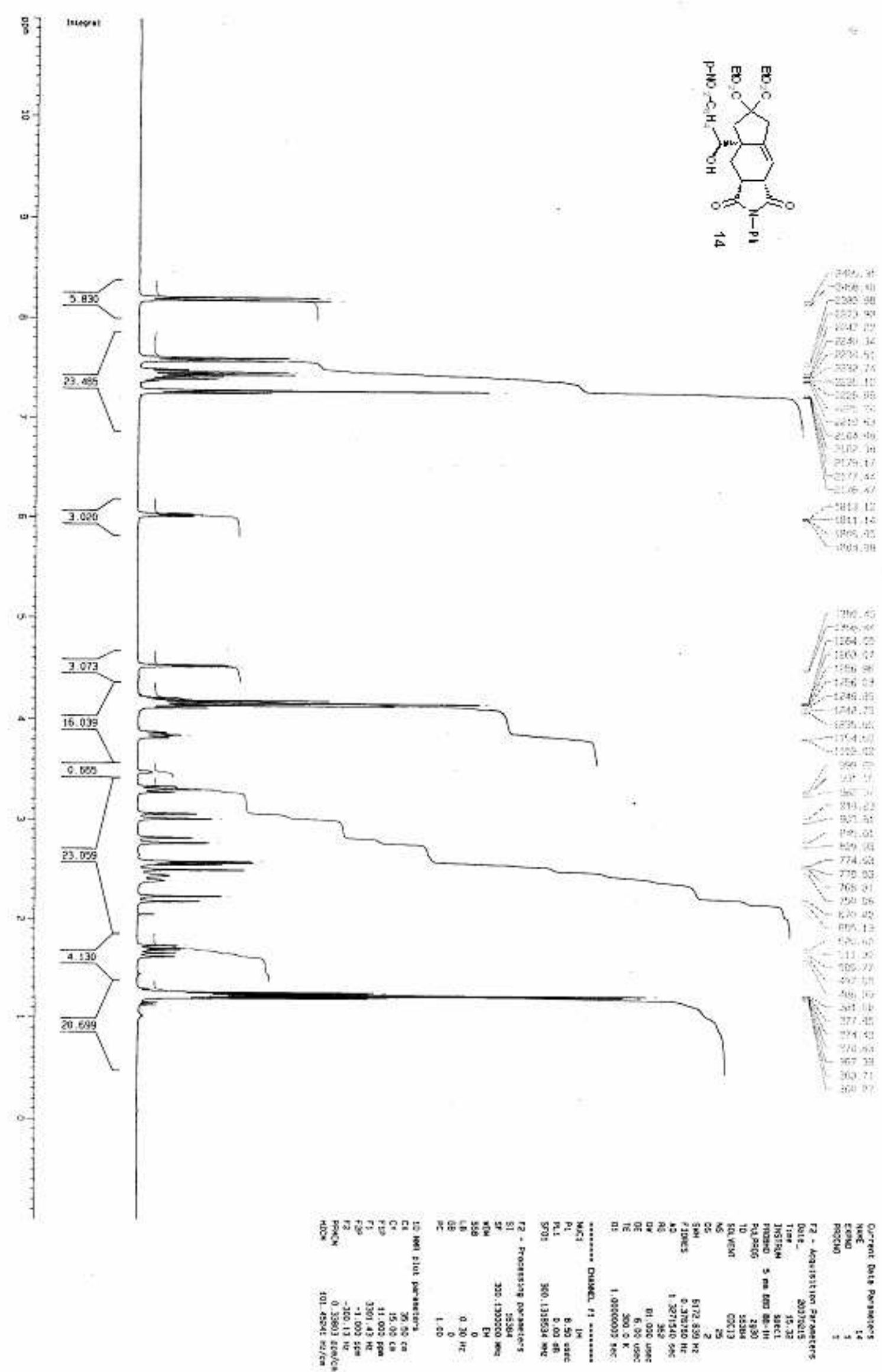


$$
1
$$




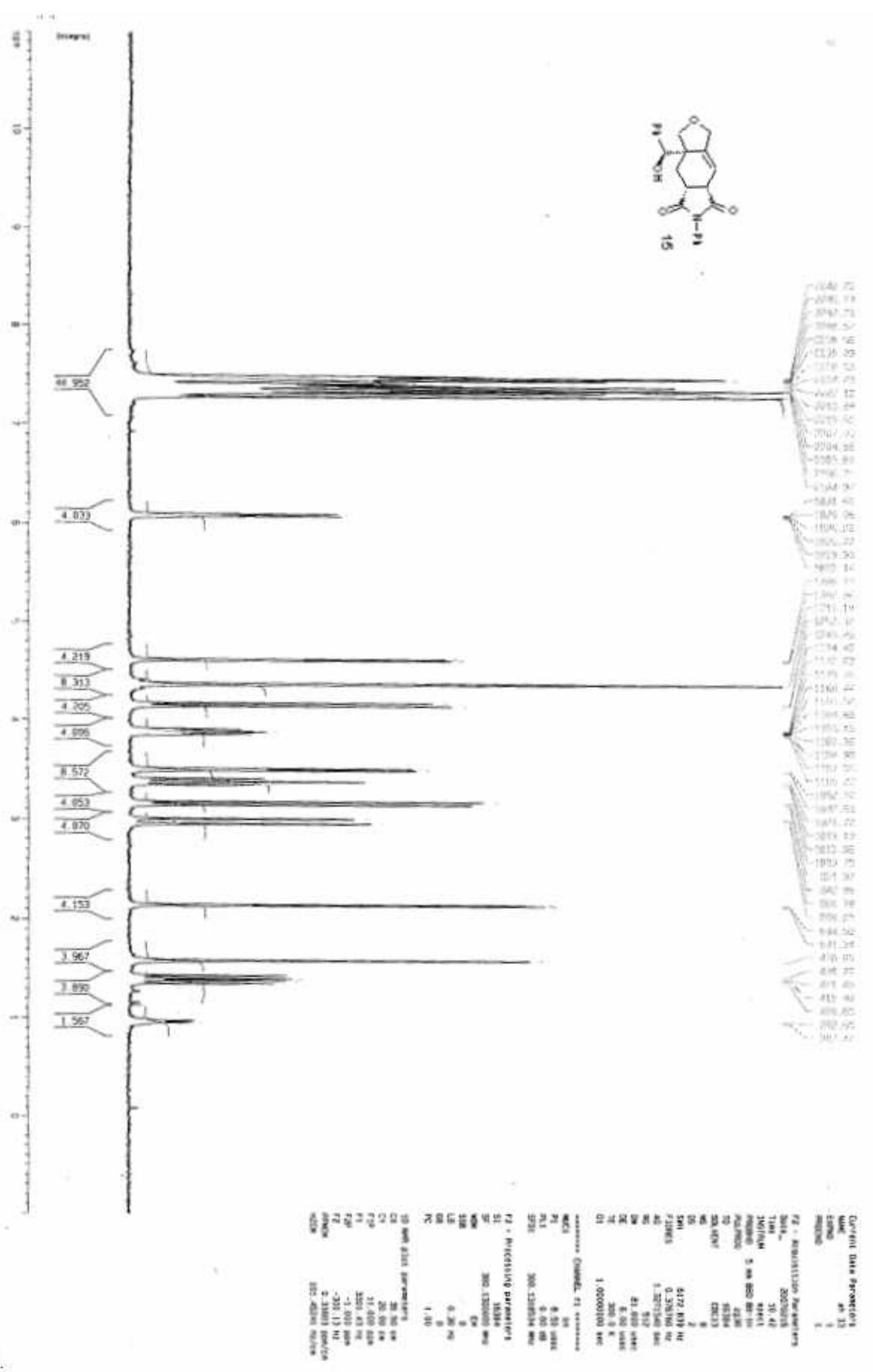




$$
\mid
$$




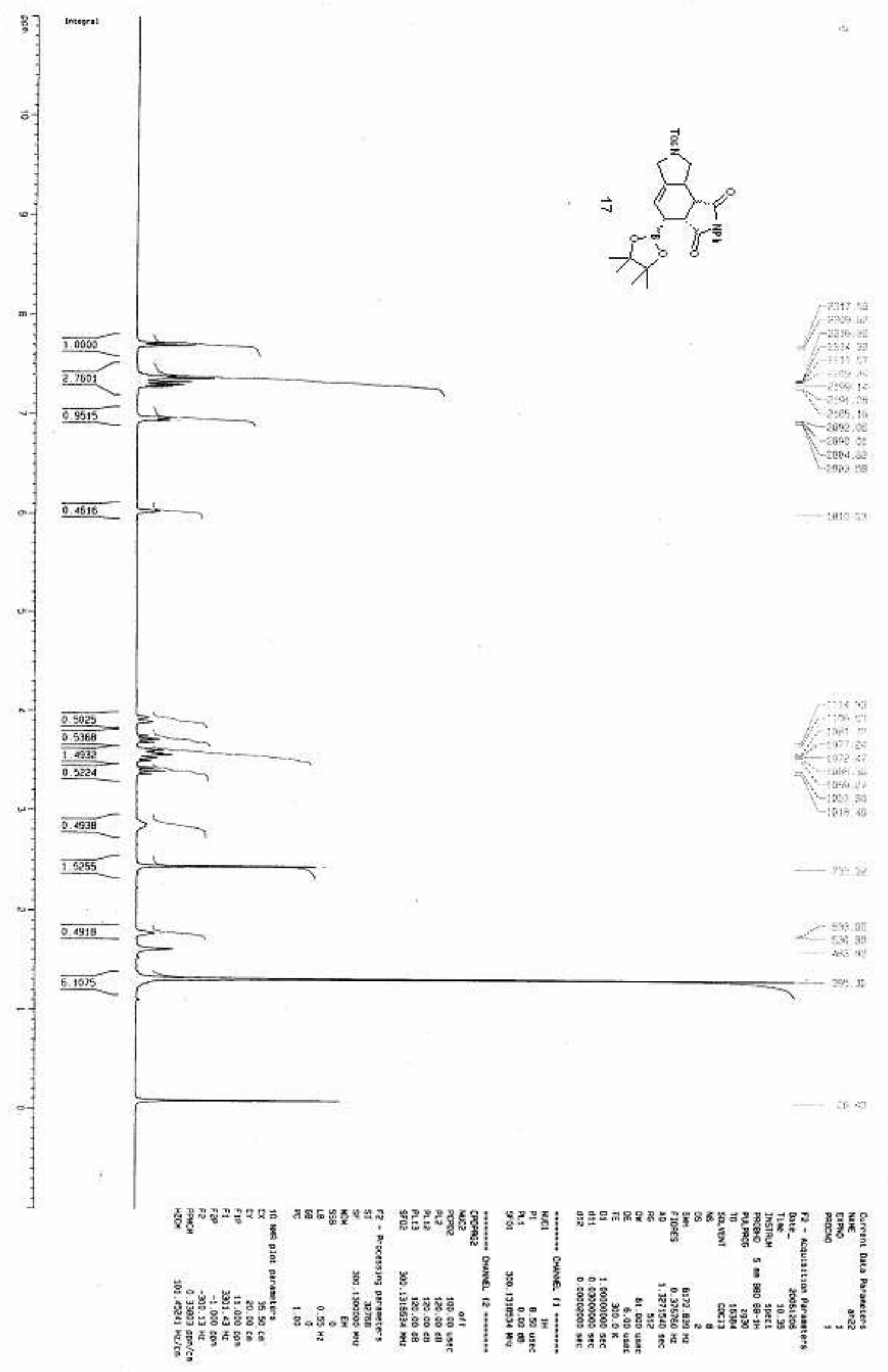




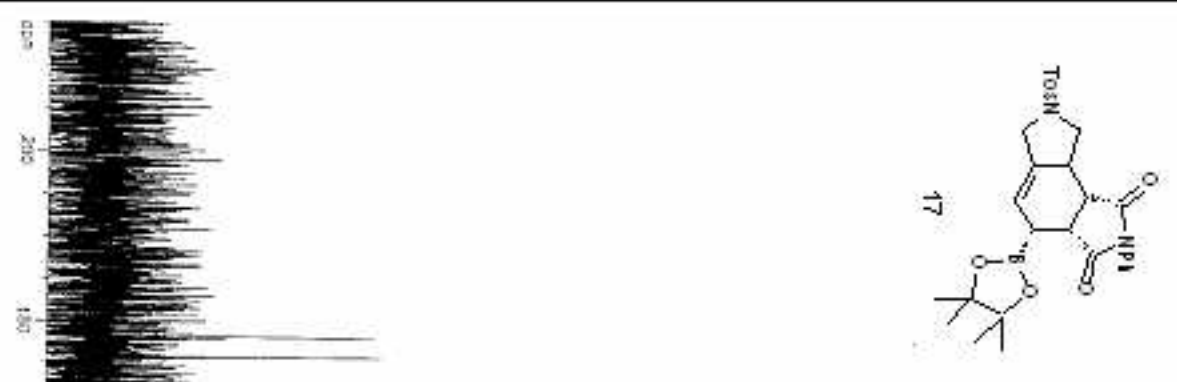

.

2

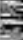

단
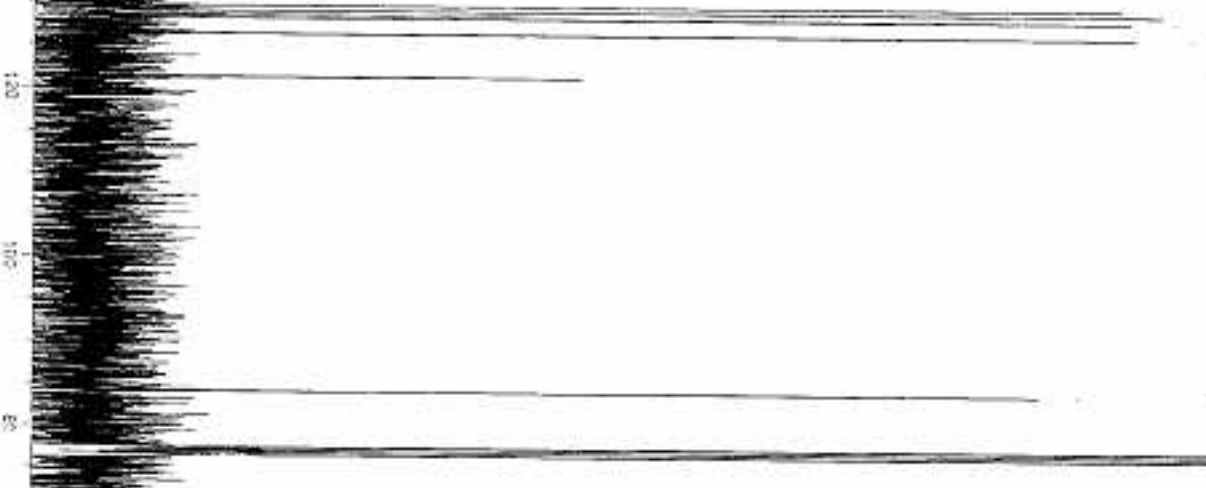

g

原

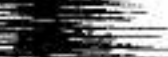

ह

풀

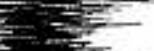

E 


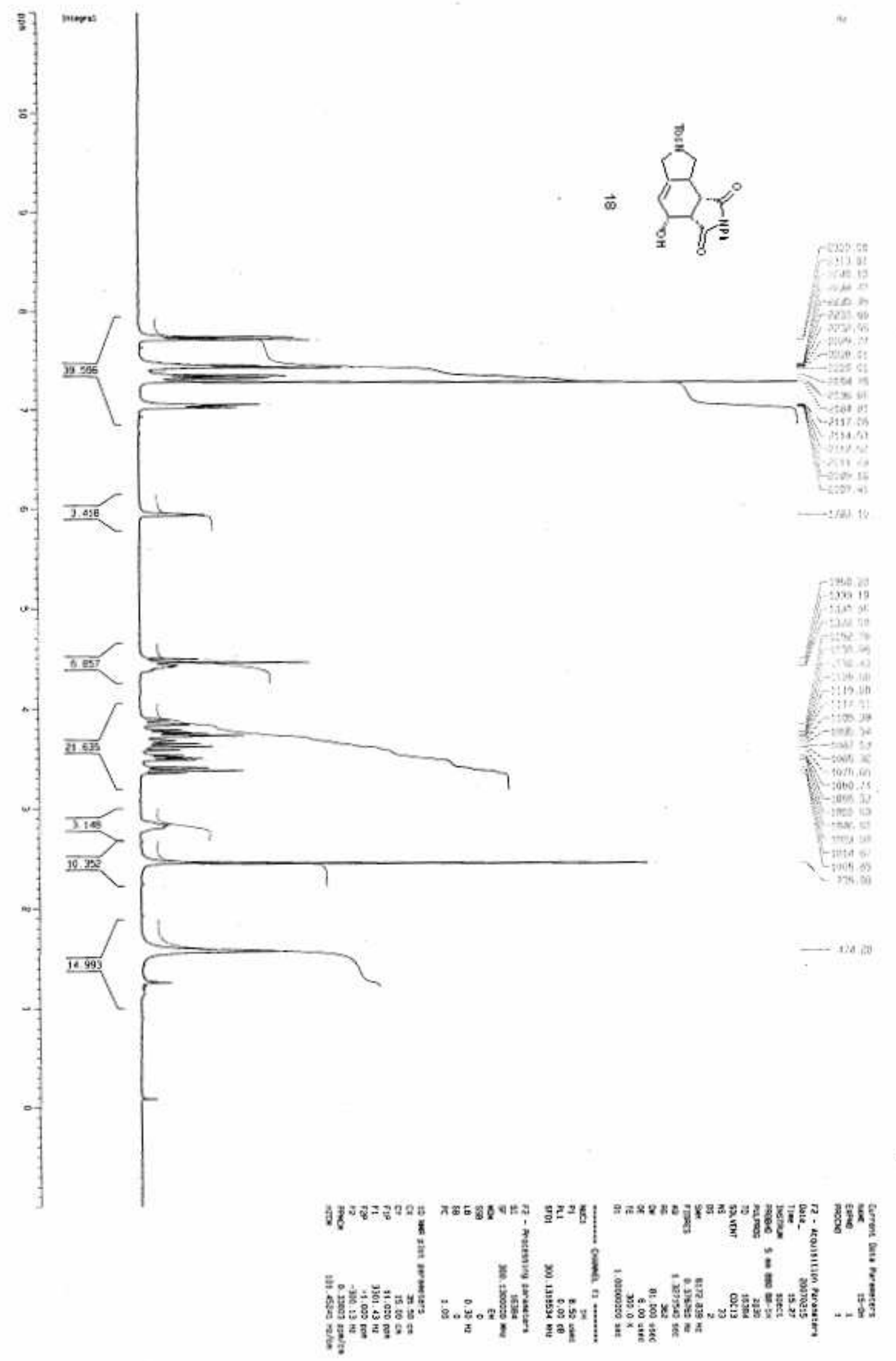




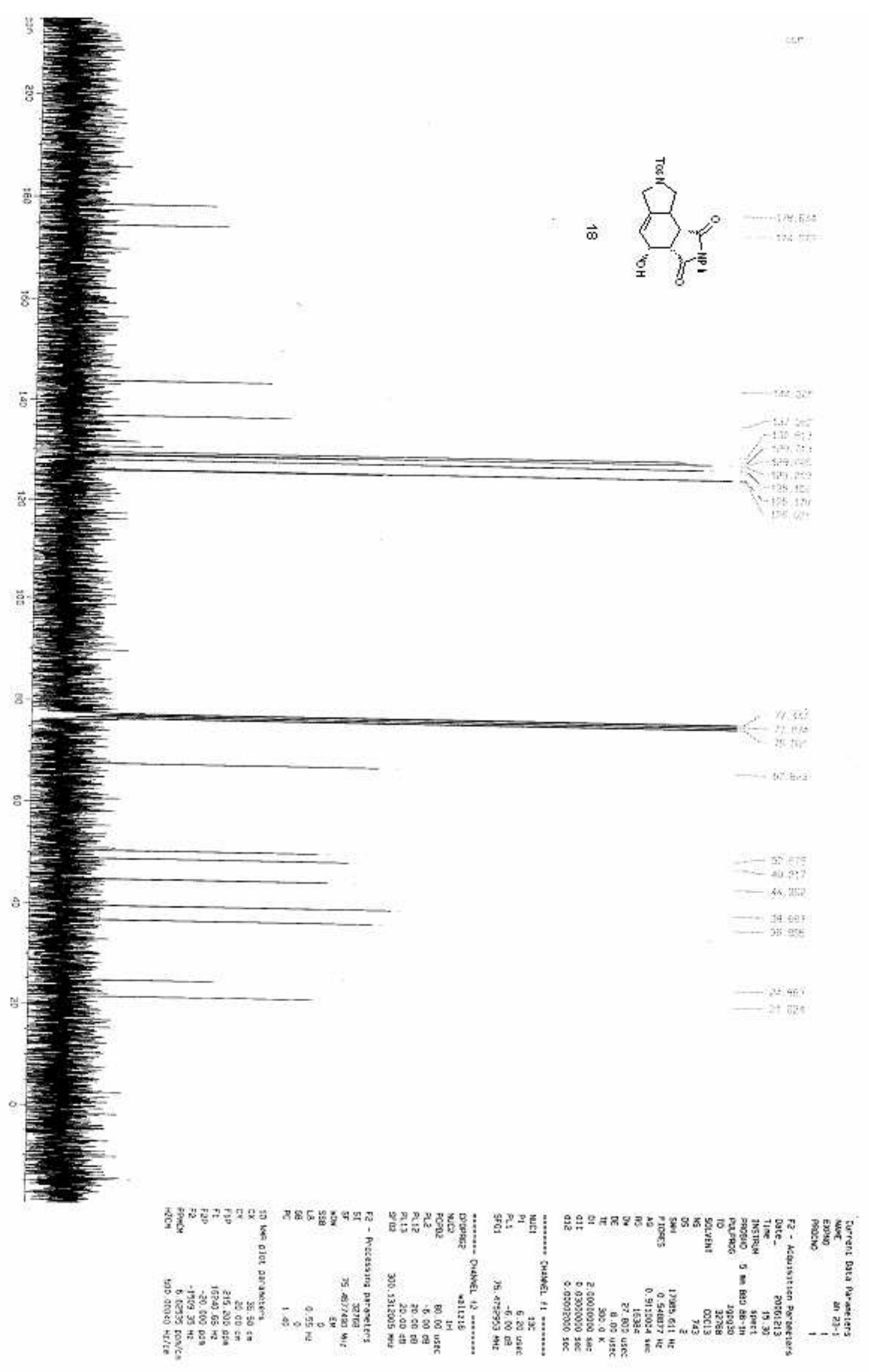

